Article

\title{
Geochemical and Geochronological Constraints on a Granitoid Containing the Largest Indosinian Tungsten (W) Deposit in South China (SC): Petrogenesis and Implications
}

\author{
Jinhua Qin *, Denghong Wang and Yuchuan Chen
}

Citation: Qin, J.; Wang, D.; Chen, Y. Geochemical and Geochronological Constraints on a Granitoid Containing the Largest Indosinian Tungsten (W) Deposit in South China (SC): Petrogenesis and Implications. Minerals 2022, 12, 80. https:// doi.org/10.3390/min12010080

Academic Editor: Maria Boni

Received: 28 November 2021 Accepted: 28 December 2021 Published: 10 January 2022

Publisher's Note: MDPI stays neutral with regard to jurisdictional claims in published maps and institutional affiliations.

Copyright: (C) 2022 by the authors. Licensee MDPI, Basel, Switzerland. This article is an open access article distributed under the terms and conditions of the Creative Commons Attribution (CC BY) license (https:// creativecommons.org/licenses/by/ $4.0 /)$.
MLR Key Laboratory of Metallogeny and Mineral Assessment, Institute of Mineral Resources, Chinese Academy of Geological Sciences, Beijing 100037, China; wangdenghong@sina.com (D.W.); hhs11234@126.com (Y.C.)

* Correspondence: qihcags@sina.cn; Tel.: +86-137-1793-1092

\begin{abstract}
Chuankou tungsten (W) ore field, with an estimated WO3 reserve exceeding 300,000 tonnes, is so far the largest Indosinian (Triassic) granite-related W ore field in South China. However, the precise emplacement ages, sources of granitoids, and their relationship with $\mathrm{W}$ mineralization are still not well understood. In this research, four main magmatic stages (G-1 to G-4) have been identified in the Chuankou ore field, including G-1 (phase I, biotite monzogranite), G-2 (phase II, two-mica monzogranite), G-3 (phase III, fine-grained granite), and G-4 (phase IV, granite porphyry). LA-ICP-MS $\mathrm{U}-\mathrm{Pb}$ dating of zircon grains from granitoids of the Chuankou $\mathrm{W}$ ore field yields emplacement ages of $230.8 \pm 1.6 \mathrm{Ma}, 222.1 \pm 0.56 \mathrm{Ma}, 203.1 \pm 1.6 \mathrm{Ma}$, and $135.5 \pm 2.4 \mathrm{Ma}$, respectively. Granitoids from the Chuankou ore field contain a large amount of peraluminous minerals such as biotite, musvite, garnet and tourmaline. Geochemically, the granitoids have high $\mathrm{Si}$ and $\mathrm{Al}(\mathrm{A} / \mathrm{CNK}>1.1)$ content but low alkali, $\mathrm{Fe}, \mathrm{Mg}, \mathrm{Mn}$, and Ca content. Moreover, there is enrichment of $\mathrm{Rb}, \mathrm{Zr}, \mathrm{Hf}$, Th, and $\mathrm{U}$, but depletions of $\mathrm{Ba}, \mathrm{Sr}, \mathrm{P}$, and $\mathrm{Ti}$. The granitoids have especially low $\mathrm{Zr}+\mathrm{Nb}+\mathrm{Ce}+\mathrm{Y}$ and high $\mathrm{Rb} / \mathrm{Ba}$ ratios, further indicating a highly fractionated S-type granite affinity with a significant crystal fractionation process in regard to K-feldspar, plagioclase, biotite, Ti-bearing minerals (except rutile), zircon, apatite, allanite, and monazite. Whole-rock $\varepsilon \mathrm{Nd}(\mathrm{t})$ and TDM2 values are -10.77 and $2090 \mathrm{Ma}$ for G-1, -9.09 to -7.47 and 1764-1684 Ma for G-2, -10.07 to -6.53 and 1669-1471 Ma for G-3, respectively, indicating that the Chuankou granitoids were derived from two episodes of partial melting of the Paleoproterozoic to Mesoproterozoic metamorphic basement. Trace elements within the zircons and whole-rock geochemistry yielded evidence of the close relationship between $\mathrm{W}$ mineralization and G-1 and G-2 granitoids of the Chuankou ore field. The batholith of the Chuankou ore field was formed 20-10 Ma later than the peak age of the collisions orogeny and formed in a post-collisional setting.
\end{abstract}

Keywords: Indosinian; Chuankou $\mathrm{W}$ ore field; Zircon U-Pb dating; highly fractionated S-type granite; post-collisional

\section{Introduction}

South China (SC) is renowned for its extensive magmatism and the giant ore deposit clusters of $\mathrm{W}, \mathrm{Sn}, \mathrm{Mo}, \mathrm{Bi}, \mathrm{Pb}, \mathrm{Zn}, \mathrm{Sb}, \mathrm{U}, \mathrm{Be}, \mathrm{Nb}, \mathrm{Ta}$, and REEs in the Yanshanian period [1-5]. These ore deposits host more than $90 \%$ of China's W resources; over $56 \%$ of global W resources [1-3]. Extensive research has been carried out around Yanshanian W mineralization and related igneous rocks using high-precision geochronological data [2,6-16]. In contrast, the Indosinian igneous rocks and $\mathrm{W}$ deposits have been not widely concerned since they are small in size and bear minimal $\mathrm{U}, \mathrm{Nb}$, and Ta deposits [17-19]. Recently, Sample reports on Indosinian W-Sn mineralization (the Miao'ershan W-Mo deposit, Hehuaping Sn deposit, Xiane'tang Sn deposit, Xitian Sn deposit, Nanyangtian W-Mo deposit, and Qingshan W deposit) have come to the forefront [2,12,20-24] (Figure 1b). Due to the unique spatial and 
temporal distribution and great metallogenic potential, increasing attention has been given to revealing the ore genesis and related granitoids of Indosinian W deposits [22,24].

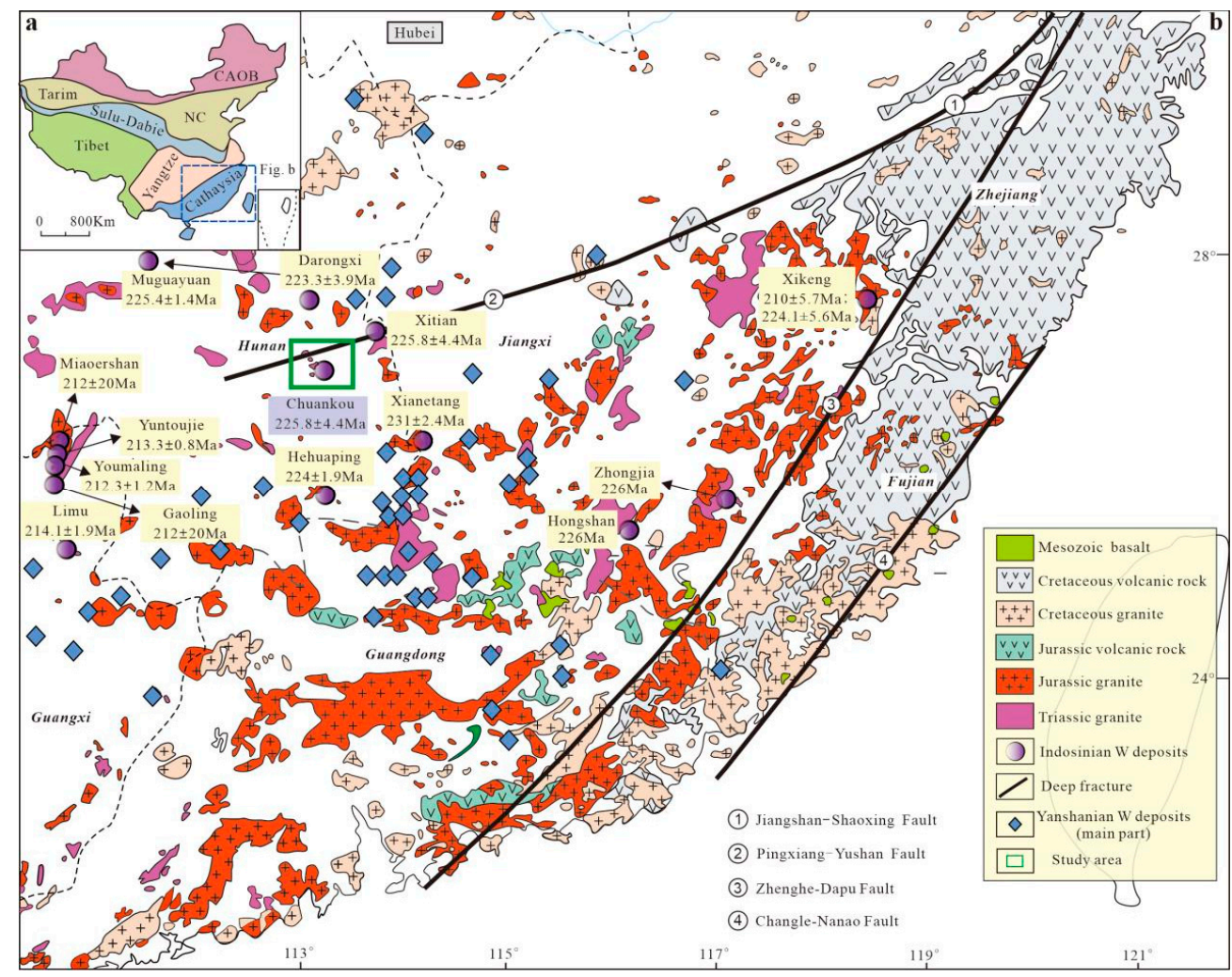

Figure 1. (a) Geological block of China and (b) Regional geological map of South China. NC, north China Block, SC, south China Block. The green box is the location of this work.

Previous research has identified the close genetic relationships between $W$ deposits and granitoids in the South China block (SCB). Numerous studies have shown that Wbearing granitoids generally present $\mathrm{S}$ - and/or A-type granitoid affinities and are enriched in $\mathrm{SiO}_{2}$ and volatiles (e.g., Li and F) [25,26]. Recently, Zhang et al. [27] and Jiang et al. [28] confirmed that $\mathrm{W}$-bearing granitoids are highly fractionated I-type granite based on the investigation of Yanshannian W deposits from Jiangxi Province and Guangdong Province. However, Huang et al. [29] proposed that W-bearing granitoids from Indosinian Yuntoujie W deposits are obvious highly fractionated S-type granite affinities. Therefore, further research is needed to solve the issue of whether the W-bearing granitoids are highly fractionated I-type or S-type.

The Chuankou W ore field is situated in the middle of the SCB and has been identified as the largest W ore field of SC with a total W metal content of over 300,000 tonnes (Figure 1a). Moreover, there are 14 important $W$ deposits distributed in the ore field (Table 1). Bai et al. [30] suggested that the host rocks of the Chuankou W deposit were formed 170 to $160 \mathrm{Ma}$. Peng et al. [31] suggested zircon U-Pb dating of host rocks to around $220 \mathrm{Ma}$ and a molybdenite Re-Os to $221 \mathrm{Ma}$ for the Sanjiaotan W deposit. However, up to now, the precise emplacement ages, sources of granitoids from the Chuankou ore field, and their relationship with $\mathrm{W}$ mineralization have been less studied and are still not well understood. 
Table 1. The mineralization characteristics of Chuankou ore field.

\begin{tabular}{ccccc}
\hline Deposit & Major Metals & Secondary Metals & Mineralization Type & Ore Grade/\% \\
\hline Chuankou & $\mathrm{W}$ & $\mathrm{Cu}, \mathrm{Bi}, \mathrm{Mo}$ & Quartz vein-type & $0.2-1.82$ \\
Baishuiling & $\mathrm{W}$ & $\mathrm{Cu}, \mathrm{Bi}, \mathrm{Mo}$ & Quartz vein-type & $0.01-1.10$ \\
Sanjiaotan & $\mathrm{W}$ & $\mathrm{Cu}, \mathrm{Bi}, \mathrm{Mo}$ & Quartz vein-type & $0.45-1.42$ \\
Huangnilong & $\mathrm{W}$ & $\mathrm{Cu}, \mathrm{Bi}, \mathrm{Mo}$ & Quartz vein-type & $1.07-4.88$ \\
Gaoritang & $\mathrm{W}$ & $\mathrm{Cu}, \mathrm{Bi}, \mathrm{Mo}$ & Quartz vein-type & $0.3-1.83$ \\
Aoshangwu & $\mathrm{W}$ & $\mathrm{Cu}, \mathrm{Bi}, \mathrm{Mo}$ & Quartz vein-type & $0.24-2.24$ \\
Liushutang & $\mathrm{W}$ & $\mathrm{Cu}, \mathrm{Bi}, \mathrm{Mo}$ & Quartz vein-type & $0.62-0.90$ \\
Tangjiangyuan & $\mathrm{W}$ & $\mathrm{Cu}, \mathrm{Mo}$ & Altered granite-type & 0.828 \\
Huanglong & $\mathrm{W}$ & $\mathrm{Cu}, \mathrm{Bi}, \mathrm{Mo}$ & Quartz vein-type & 0.65 \\
Wubeichong & $\mathrm{W}$ & $\mathrm{Cu}, \mathrm{Bi}, \mathrm{Mo}$ & Quartz vein-type & $0.01-0.44$ \\
Yanglinao & $\mathrm{W}$ & $\mathrm{Cu}, \mathrm{Bi}, \mathrm{Mo}$ & Quartz vein-type & 0.47 \\
Maowan & $\mathrm{W}$ & $\mathrm{Cu}, \mathrm{Mo}$ & Altered granite-type & $250 \mathrm{~g} / \mathrm{m}^{3}$ \\
Tangjiawan & $\mathrm{W}$ & $\mathrm{Sn}$ & Placer-type & $0.079-0.399$ \\
Baishuiling & $\mathrm{W}$ & $\mathrm{Cu}, \mathrm{Bi}, \mathrm{Mo}$ & Quartz vein-type & \\
\hline
\end{tabular}

In this study, we present new zircon U-Pb ages, major and trace element characteristics, $\mathrm{Sr}-\mathrm{Nd}$ isotopic compositions of granitoids from the Chuankou $\mathrm{W}$ ore field to constrain the source, magmatic genesis, and their relationships with $\mathrm{W}$ deposits.

\section{Regional Geology}

The Chuankou ore field is situated in the middle-eastern part of Hunan Province, the margin of the Neoproterozoic suture zones between the Yangtze Block and Cathaysia Block - the Qin-Hang suture zone (Figure 1a). The strike of the Qin-hang suture zone yields an NE-SW orientation along the Hangzhou Bay in Zhejiang Province to Qinzhou Bay in Guangxi Province, approximately $2000 \mathrm{~km}$ in length and 100-150 km in width [32-34]. The Qin-Hang suture zone is also a giant $\mathrm{W}-\mathrm{Sn}-\mathrm{Mo}-\mathrm{Bi}-\mathrm{Cu}-\mathrm{Pb}-\mathrm{Zn}-\mathrm{g}-\mathrm{Au}-\mathrm{U}$ polymetallic mineralization zone of the SCB [35] (Figure 1b). During the Neoproterozoic period, this area underwent a collision between the Yangtze Block and Cathaysia Block caused by the closure of the Paleo-South China Sea. The subsequent intracontinental fold orogeny continued to influence the region during the early Paleozoic period. Due to the northward subduction of the Indo-China block and closure of the ancient Tethys Ocean in the late Paleozoic to early Mesozoic, the tectonic regime transitioned to multiplate convergence and caused E-W-trending folded orogenic belts and foreland basins [9,36-40].

Various metal resources are distributed throughout the Chuankou ore field, including $\mathrm{W}, \mathrm{Sn}, \mathrm{Cu}, \mathrm{Nb}, \mathrm{Ta}, \mathrm{Fe}, \mathrm{Pb}, \mathrm{Zn}$, and $\mathrm{Au}$ (Table 1). The main types of $\mathrm{W}$ deposits in the Chuankou ore field are altered granite-type scheelite, quartz vein-type wolframite, and veinlet-disseminated scheelite. The altered granite-type scheelite is mainly developed in the Maowan deposit, Tangjiangyuan deposit, and Baishui deposit. Wolframite and molybdenite grains are disseminated in altered two-mica monzogranites. The quartz vein-type wolframite is mainly developed in the Sanjiaotan deposit, Huanglong deposit, and Nanwan deposit. The main associated metals involve $\mathrm{Cu}, \mathrm{Bi}, \mathrm{Mo}, \mathrm{Pb}$, and $\mathrm{Zn}$. The vein-disseminated scheelite is mainly distributed in the Yanglinao deposit (Figure 2) [41]. 


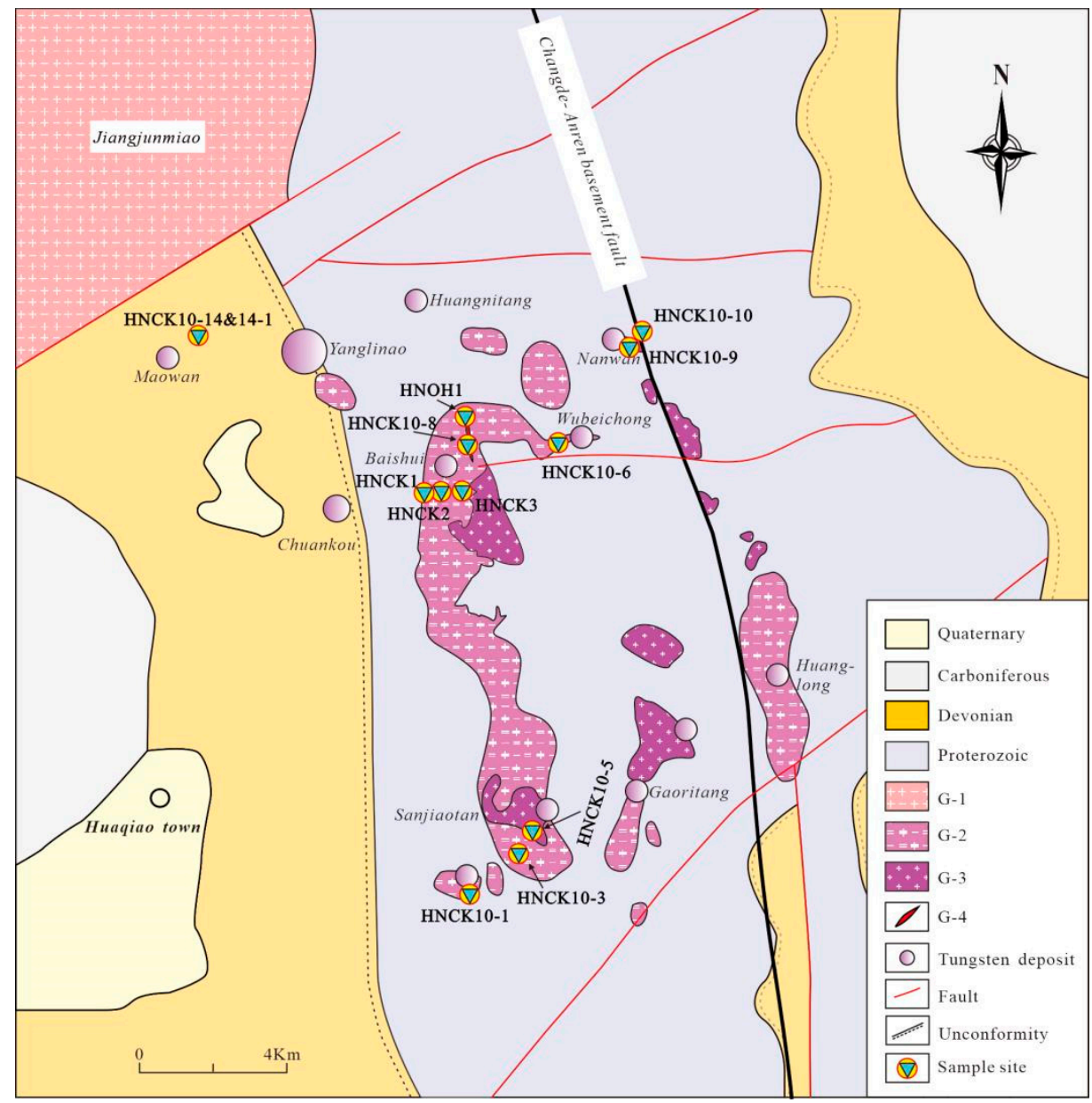

Figure 2. Geological map of Chuankou tungsten ore field.

\section{Ore Deposit Geology}

The Proterozoic metamorphic basement exposed in the center of the ore field contains a metamorphic silty slate and an argillaceous slate of the Neoproterozoic Wuqiangxi Formation of Banxi group. These are the most important host rocks of the quartz veintype wolframite. The Paleozoic strata are exposed in the margin of the ore field and are unconformably covered above the metamorphic basement. It is composed of siliceous sedimentary breccia and shale with of the Devonian Yanglinao Formation (D2y), shale of the Carboniferous Yanguan Formation (C1y), and the diluvial layer of the Quaternary. Among them, the siliceous sedimentary breccia of Yanglinao Formation (D2y) has been confirmed as one of the wall rocks of the vein-type scheelite in the Yanglinao deposit (Figure 2). The Chuankou W ore field is exposed in the core of the Chuankou uplift, which is composed of a series of anticlines. The Chuankou uplift belongs to the eastward extension of the Qiyangshan zigzag-shaped structural ridge axis. Two groups of folds were developed: (1) the early E-W-direction fold belt and (2) the late N-S-direction fold belt. Fault structures in the ore field are oriented mainly in an NNW direction and NEE direction. The ENE-direction fault clusters are early faults that occur near the internal contact zone between the granitoids and surrounding rocks. The NNW-direction fault clusters are deep normal faults, which control the ore body's occurrences, orientation and enrichment (Figure 2).

Granitoids of the Chuankou ore field are exposed in the core of the Chuankou uplift with an area of $15 \mathrm{~km}^{2}$. According to fieldwork in this research, four main magmatic stages could be observed (Figure 2). The emplacement sequence is biotite monzogranite (G-1) $\rightarrow$ two-mica monzogranite (G-2) $\rightarrow$ fine-grained granite (G-3) $\rightarrow$ granite porphyry (G-4) (Figure 3a-d). 


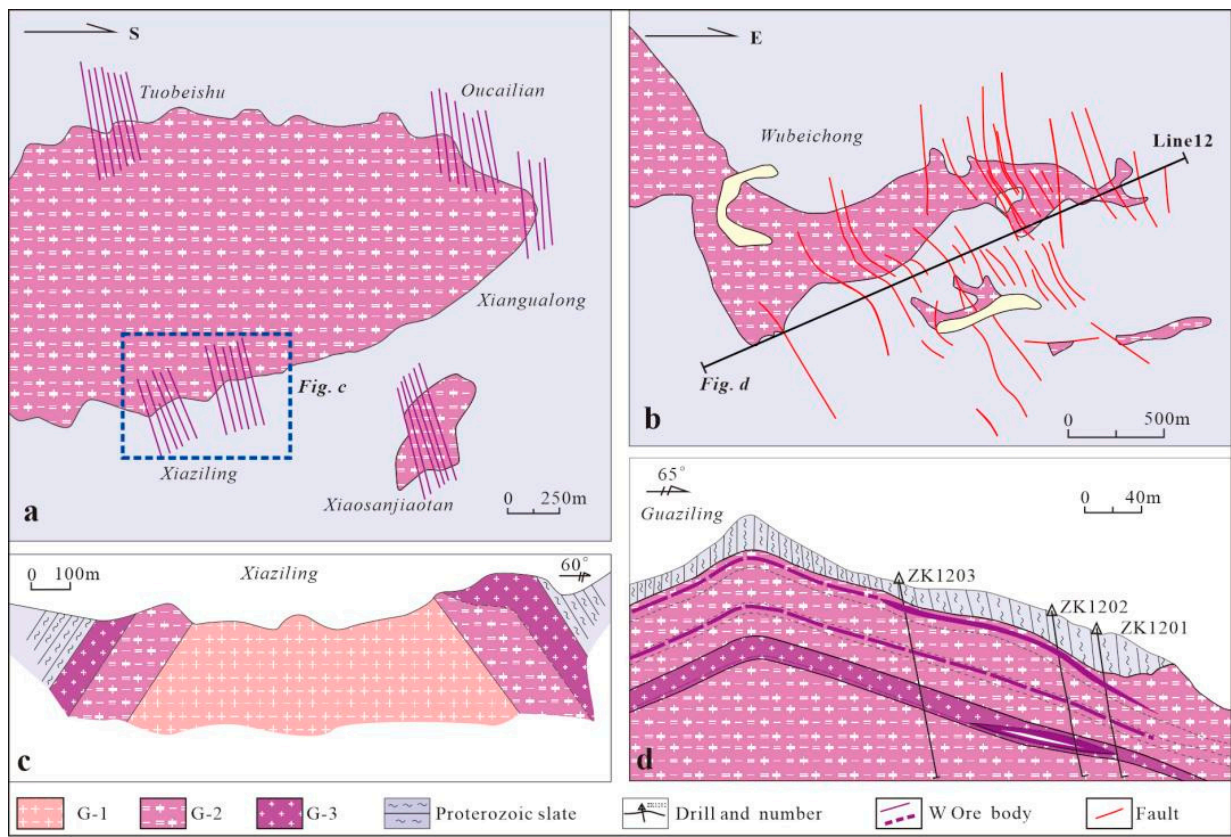

Figure 3. (a) Geological map of Sanjiatan deposit, (b) Geological map of Wubeichong deposit, (c) Geological section map of Xiazilin ore block in Sanjiatan deposit, (d) Line 12 profile map of Wubeichong deposit.

(1) Biotite monzogranites (G-1) are exposed at the Maowan and Baishui deposits. The main minerals assemblage includes quartz (25 to $30 \mathrm{vol} . \%$ ), plagioclase (40 vol. $\%$ ), and K-feldspar ( 25 vol.\%). The secondary minerals are biotite (5-10 vol.\%) and muscovite (1-5 vol.\%). Accessory minerals include magnetite/ilmenite, zircon, apatite, and xenotime. Biotite appears light brown to dark brown with sizes ranging from 200 to $500 \mu \mathrm{m}$, whereas the diameters of quartz and feldspar are approximately $2-5 \mathrm{~mm}$ (Figure $4 \mathrm{e}, \mathrm{g}$ ).

(2) Two-mica monzogranite (G-2) is the main component, accounting for four out of five exposed areas of the Chuankou granitoids. These monzogranites have a mediumto coarse-grained structure and contain quartz (30\%), sodium feldspar (30\%), K-feldspar $(20 \%)$, muscovite $(10 \%)$, and biotite $(5 \%)$. Garnet, uraninite, xenotime, and zircon are common accessory minerals with contents of $1-3 \%$. Euhedral to hypidiomorphic crystal molybdenite (1-2 mm), columnar wolframite ( $5 \mathrm{~mm})$, and scheelite (0 to $1 \mathrm{~mm}$ ) occur in the greisen belt, which developed in the shallow part of G-2 (Figure $4 \mathrm{a}, \mathrm{b}, \mathrm{d}, \mathrm{h}-\mathrm{j}$ ).

(3) Fine-grained granite (G-3) is widely exposed at the region and intrudes into the G-2 and metamorphic slate as veins about $30-50 \mathrm{~cm}$ in width. G-3 is dark to gray in color and has a fine-grained texture. The minerals assemblage includes quartz, plagioclase, K-feldspar, and muscovite. Generally, the mineral crystals of G-3 are smaller than $0.5 \mathrm{~mm}$. Slight alteration were developed in K-feldspar crystals (Figure $4 b, c, 1)$.

(4) Granite porphyry (G-4) is only exposed on the north side of Chishui Village roads. It occurs as a vein and intrudes into G-2 with a width of $15-20 \mathrm{~m}$. G-4 exhibits a large structure and porphyritic texture. The phenocrysts (approximately $30 \mathrm{vol} . \%$ of the whole rocks) are $0.5-2 \mathrm{~mm}$ in size and composed of quartz (30 vol.\% of total phenocrysts), potassium feldspar (60 vol.\% of total phenocrysts), and a small amount of plagioclase and muscovite (less than 10 vol.\%). The matrix is microgranular, which occupies 70 vol.\% of all rocks (Figure 4f,k). 


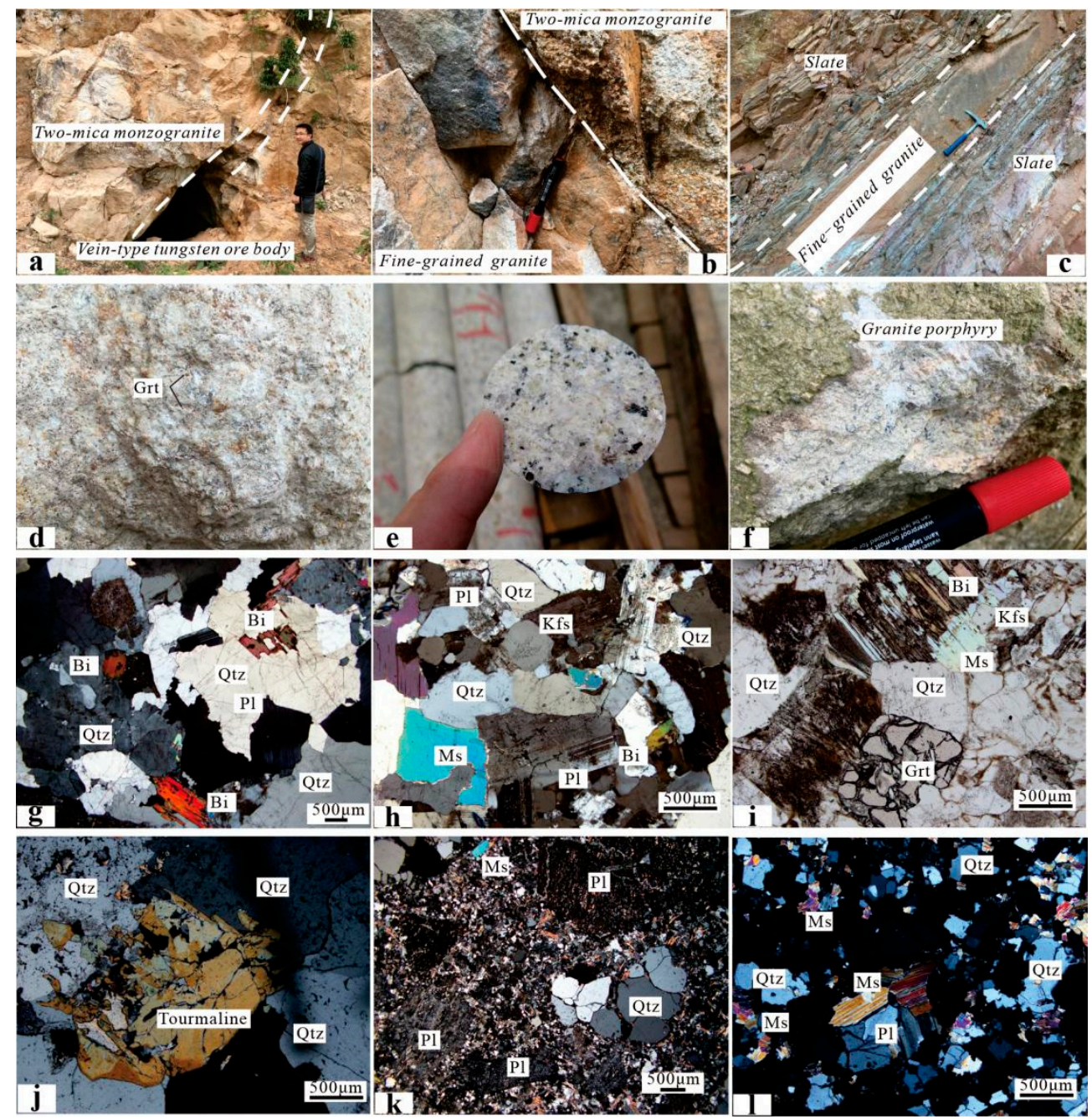

Figure 4. Petrographic photographs of intrutions in Chuankou ore field. (a) the wolframite- quartz vein in coarse two-mica monzogranite(G-2), (b) Fine-grained granite (G-3) intrude into coarse twomica monzogranite, (c) Fine-grained granite (G-3) intrude into shallow metamorphic slate, (d) Disseminated spessartine developed in two-mica monzogranite(G-3), (e,g) Biotite monzogranite(G-1), $(\mathbf{f}, \mathbf{k})$ Granite porphyry(G-4), (h-j) Two-mica monzogranite(G-2), (1) Fine-grained granite(G-3). Bibiotite, Pl- plagioclase, Kfs- K-feldspar, Ms- muscovite, Qtz- quartz, Grt- garnet.

\section{Alteration and Mineralization}

Field observation shows that hydrothermal alteration occurred in the contact zone between the granitoids and Neoproterozoic strata and its adjacent area. The alteration types contain silicification, greisenization, potash feldspathization, tourmalinization, carbonatization, argillization. Greisenization, and silicification as the main high-temperature hydrothermal alterations that are widely developed at the top of the contact zones between the G-2 and Neoproterozoic strata. In addition, greisenization occurred intensely along the margins between barren or fertile quartz veins. The interior of the veins developed potassium feldspar, tourmaline, and calcite.

The mineralization types of the Chuankou ore field include altered granite-type scheelite and molybdenite, quartz vein-type wolframite, and veinlet-disseminated-type scheelite. Among them, the altered granite-type scheelite and molybdenite occur in the top greisenization zone of two-mica monzogranites (Maowan, Hubeichong, and Baishui deposits); generally, low ore grades and limited spatial scales. Quartz vein-type wolframite occurs in the fault zone above the granitoids (Nanwan and Hunaglong deposits). Ore-bearing veins are along the NNE direction, and the angle of inclination is $70^{\circ}$ to $80^{\circ}$. Veinlet-disseminated 
scheelite has economic value only in the Yanglinao deposit, and it occurs in the siliceous breccia belt $\left(D_{2} y\right)$ as a mesh vein structure.

(1) Ore minerals assemblage is composed of wolframite, cassiterite, molybdenite, scheelite, chalcopyrite, sphalerite, arsenopyrite, pyrite, molybdenite, and uraninite. Gangue minerals include quartz, calcite, muscovite, tourmaline, fluorite, chlorite, garnet, barite, topaz, and tourmaline. Based on the mineral relationships, characteristics of alteration, and mineralization, four phases and five stages of mineralization processes have been generally identified (Figure 5).

\begin{tabular}{|c|c|c|c|c|c|}
\hline $\begin{array}{l}\text { Metallogenic } \\
\text { epoch }\end{array}$ & $\begin{array}{l}\text { Pneumatolytic } \\
\text { hydrothermal }\end{array}$ & $\begin{array}{r}\text { Middle to his } \\
\text { hydro }\end{array}$ & $\begin{array}{l}\text { h-temperature } \\
\text { thermal }\end{array}$ & $\begin{array}{l}\text { Middle to low- } \\
\text { temperature } \\
\text { hydrothermal }\end{array}$ & $\begin{array}{l}\text { Low-temperature } \\
\text { hydrothermal }\end{array}$ \\
\hline Stage & $\begin{array}{l}\text { Greisenization } \\
\text { stage }\end{array}$ & $\mid \begin{array}{c}\text { Quartz-Wolframite } \\
\text {-Molybdenite stage }\end{array}$ & $\begin{array}{l}\text { Quartz-Wolframite- } \\
\text { Molybdenite } \\
\text { (Bismuthinite)- } \\
\text { Scheelite stage }\end{array}$ & $\begin{array}{l}\text { Quartz-polymetallic } \\
\text { sulfide stage }\end{array}$ & $\begin{array}{l}\text { Quartz - fluorite } \\
\text { - calcite stage }\end{array}$ \\
\hline Wolframite & $\longrightarrow$ & & & & \\
\hline Scheelite & & & & & \\
\hline Molybdenite & & & & & \\
\hline Bismuthinite & & & & & \\
\hline Chalcopyrite & & & & & \\
\hline Pyrite & & & & & \\
\hline Sphalerite & & & & & \\
\hline Galena & & & & & \\
\hline Arsenopyrite & & & & & \\
\hline Uraninite & & & & & \\
\hline Tourmaline & & 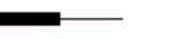 & & & \\
\hline Orthoclase & & & & & \\
\hline Muscovite & & & & & \\
\hline Quartz & & & & & \\
\hline Fluorite & & & & & \\
\hline Calcite & & & & & \\
\hline
\end{tabular}

Figure 5. The mineral paragenetic sequence of the Chuankou ore field.

(2) The medium- to high-temperature hydrothermal period is the main metallogenic stage and includes the early stages of the quartz vein-wolframite-molybdenite assemblage and the late stage of the quartz-wolframite-molybdenite (bismuthinite)-scheelite assemblage. During the mineralization process, scheelite and molybdenite crystallized slightly 
later than wolframite; The tabular wolframite crystals were widely filled by scheelite. Minerals assemblage includes wolframite, molybdenite, scheelite, bismuthinite, and pyrite (less), and a small amount of chalcopyrite (Figure $6 \mathrm{~b}-\mathrm{d}, \mathrm{f}-\mathrm{h}$ ).
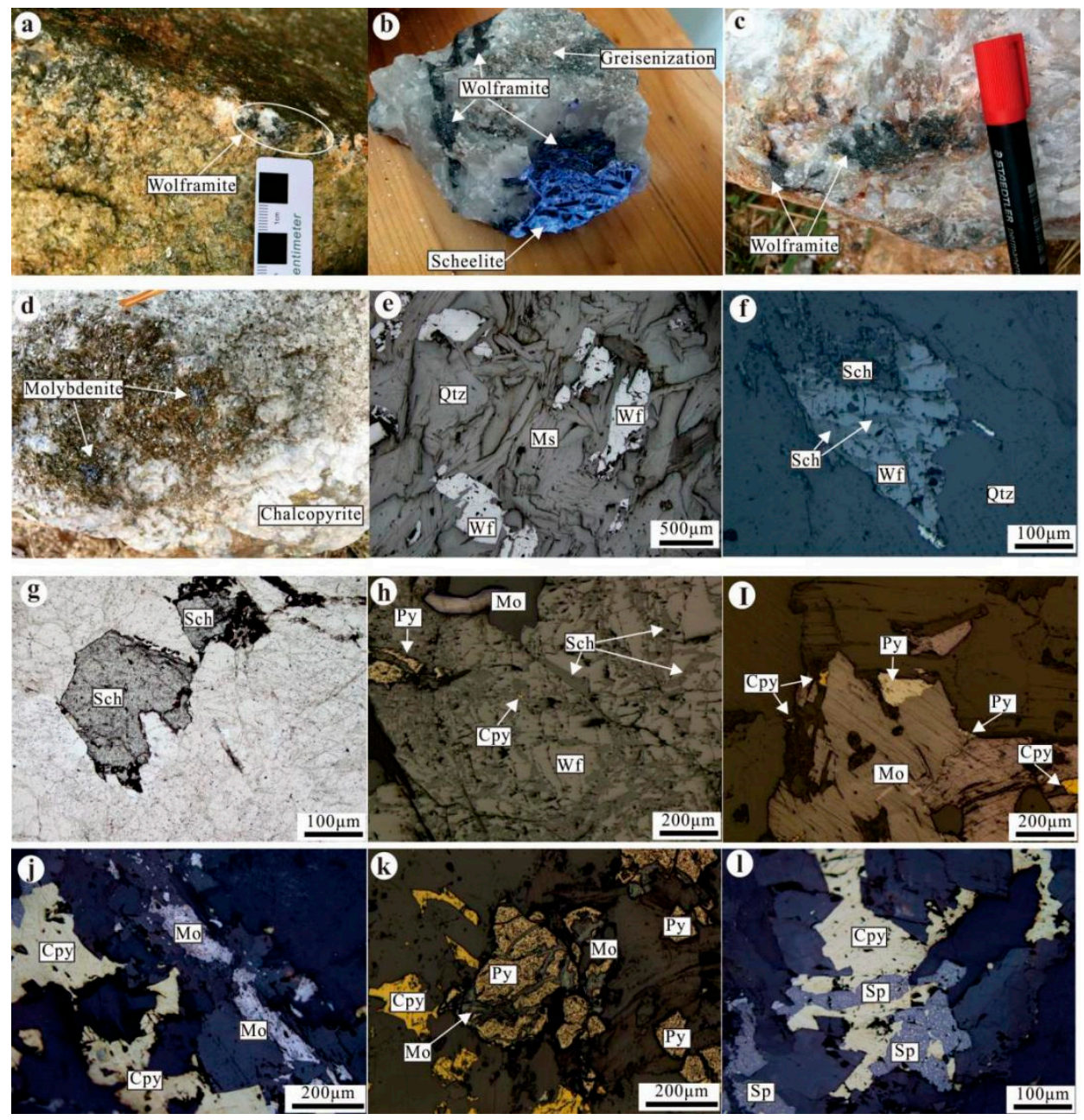

Figure 6. $(\mathbf{a}-\mathbf{c}, \mathbf{f}-\mathbf{h})$ Ore and photomicrograph of quarz-wolframite vein from Chuankou ore field, $(\mathbf{d}, \mathbf{e})$ Ore and photomicrograph of alterated granite type wolframite. (a-h), Medium to high temperature hydrothermal mineralization stage, (i-1), Medium to low temperature hydrothermal mineralization stage. Sch- scheelite; Wf- wolframite; Ms- muscovite; Py- pyrite, Cpy- chalcopyrite, Spsphalerite, Mo- molybdenite, Qtz- quartz.

(3) The low- to middle-temperature hydrothermal period. The quartz and sulfide stage shows no obvious mineralization of $\mathrm{W}$. The minerals assemblage is composed by chalcopyrite, sphalerite, pyrite, and arsenopyrite. (Figure 6i-1)

(4) Low-temperature hydrothermal period. Low-temperature minerals (fluorite and calcite) and a small amount of sulfide (sphalerite and galena) are the dominant minerals in this period.

\section{Sampling and Method}

\subsection{Sampling}

Thirteen samples were collected for the whole-rock geochemical analysis (HNCK1, 2, 3, 10-1, 10-3, 10-5, 10-6, 10-8, 10-9, 10-10, 10-14, 14-1, and HNOH1). Samples HNCK1, 2, 3 , 10-6, 10-8, and HNOH1 were collected in the Baishui deposit, HNCK1 and 2 are altered two-mica monzogranite (G-2), and HNCK3 and 10-6 are fresh two-mica monzogranite (G-2). HNCK10-8 and HNOH1 are granite porphyry samples (G-4). HNCK10-1, 10-3, and 
10-5 were collected from the Sanjiaotan deposit (HNCK10-1 and 10-3 are altered two-mica monzogranite (G-2), and HNCK10-5 is fine-grained granite (G-3)). HNCK10-9 and 10-10 are fine-grained granite (G-3) from the Nanwan deposit, and HNCK10-14 and 14-1 are biotite monzogranites (G-1) from the Manwan deposit. Four out of thirteen samples were selected carefully for LA-ICP-MS zircon U-Pb analysis (HNCK2, 10-8, 10-10, 10-14), and 6 out of 13 samples were selected for whole-rock Rb-Sr and Sm-Nd isotopic composition analysis (HNCK1, 2, 3, 10-5, 10-9, and 10-14).

\subsection{Geochoronology}

Zircon grains were separated for $\mathrm{U}-\mathrm{Pb}$ age dating at the Langfang Regional Geology and Mineral Resources Survey Institute. The bulk samples were crushed to 60-80 mesh size, and zircons were separated using gravity and electromagnetic techniques and hand-picked under a binocular microscope. The samples were then mounted on epoxy resin, smoothed and polished, and finally gold coated. The zircons were examined using transmitted and reflected light and cathodoluminescence (CL) microscopy. Zircon $\mathrm{U}-\mathrm{Pb}$ dating was performed at the Institute of Mineral Resources, CAGS, Beijing, using a Finnigan Neptune inductively coupled plasma mass spectrometer (MC-ICP-MS) with a new wave UP213 laserablation system. Helium was used as the carrier gas, and the beam diameter was $30 \mu \mathrm{m}$ with a $10 \mathrm{~Hz}$ repetition rate and laser power of $2.5 \mathrm{~J} / \mathrm{cm}^{2}$. Eight ion counters were used to simultaneously receive the ${ }^{238} \mathrm{U},{ }^{235} \mathrm{U},{ }^{232} \mathrm{Th},{ }^{208} \mathrm{~Pb},{ }^{207} \mathrm{~Pb},{ }^{206} \mathrm{~Pb},{ }^{204} \mathrm{~Pb}$, and $202 \mathrm{Hg}$ signals, whereas data for ${ }^{208} \mathrm{~Pb},{ }^{232} \mathrm{Th},{ }^{235} \mathrm{U}$, and ${ }^{238} \mathrm{U}$ were collected on a Faraday cup. Zircon GJ-1 was used as standard, and Plešovice zircon was used to optimize the mass spectrometer. $U$, $\mathrm{Th}$, and $\mathrm{Pb}$ concentrations were calibrated using $29 \mathrm{Si}$ as an internal standard and zircon M127 (U: 923 ppm; Th: 439 ppm; Th/U: 0.4750) as an external standard [42]. ${ }^{207} \mathrm{~Pb} /{ }^{206} \mathrm{~Pb}$, and ${ }^{206} \mathrm{~Pb} /{ }^{238} \mathrm{U}$ were calculated using the ICP-MS DataCal 4.3 program. Common Pb was not corrected because of high ${ }^{206} \mathrm{~Pb} /{ }^{204} \mathrm{~Pb}$. Abnormally high ${ }^{204} \mathrm{~Pb}$ data were deleted. The Plešovice zircon was dated as unknown and yielded a weighted mean ${ }^{206} \mathrm{~Pb} /{ }^{238} \mathrm{U}$ age of $337 \pm 2 \mathrm{Ma}(2 \mathrm{SD}, \mathrm{n}=12)$, which is in good agreement with the recommended ${ }^{206} \mathrm{~Pb} /{ }^{238} \mathrm{U}$ age of $337.13 \pm 0.37 \mathrm{Ma}$ (2SD) [43]. Age calculations were performed, and Concordia diagrams were generated using the Isoplot/Ex 3.0 software [44].

\subsection{Geochemistry}

Whole-rock major, trace, and rare earth element concentrations were analyzed at the National Geological Experiment Test Center, Beijing. Whole-rock major, trace, and rare earth element concentrations were analyzed at the National Geological Experiment Test Center, Beijing. Whole-rock major elements were analyzed using a plasma spectrometer (PE8300). All results were normalized against the Chinese rock reference standard JY/T0151996 [45]. The analytical uncertainties were less than $\pm 2 \%$.

\subsection{Sr-Nd Isotope}

Fresh samples were ground with an agate mill and powders were spiked with mixed isotope tracers, dissolved in Teflon capsules with $\mathrm{HF}+\mathrm{HNO}_{3}$ acid, and separated by conventional cation-exchange techniques. The isotopic measurements were performed on a VG-354 mass spectrometer at the Institute of Geology and Geophysics, Chinese Academy of Sciences [46]. The mass fractionation corrections for $\mathrm{Sr}$ and $\mathrm{Nd}$ isotopic ratios were based on ${ }^{86} \mathrm{Sr} /{ }^{88} \mathrm{Sr}=0.1194$ and ${ }^{146} \mathrm{Nd} /{ }^{144} \mathrm{Nd}=0.7219$. Repeat analyses yielded an ${ }^{87} \mathrm{Sr} /{ }^{86} \mathrm{Sr}$ ratio of $0.71023 \pm 0.00006$ for the NBS-987 $\mathrm{Sr}$ standard and an ${ }^{143} \mathrm{Nd} /{ }^{144} \mathrm{Nd}$ ratio of $0.511845 \pm 0.000012$ for the La Jolla standard. Detailed descriptions of the analytical techniques can be found elsewhere-in [47] and references therein.

\section{Results}

\subsection{Chronology}

(1) G-2: Zircon grains are columnar crystals with sizes from 150 to $200 \mu \mathrm{m}$ (Figure 7). $\mathrm{CL}$ images have shown that zircons have typical oscillatory magmatic zoning. $\mathrm{Pb}$ content 
ranges from 12.1 to $124 \mathrm{ppm}$, Th content ranges from 67.82 to $219.30 \mathrm{ppm}$, and U content ranges from 506.25 to $1187.55 \mathrm{ppm}$ (Table 2). Out of 30 analyzed spots of the biotite monzogranites, 14 spots yield ${ }^{206} \mathrm{~Pb} /{ }^{238} \mathrm{U}$ ages of $215.9 \pm 4.54$ to $231 \pm 2.19 \mathrm{Ma}$, and the obtained zircons have a concordance age of 222.1 $\pm 0.56 \mathrm{Ma}(\mathrm{MSWD}=2.8$ ) (Figure 8a).

(2) G-1: The length/width ratios of zircons are close to 1-2. The sizes of zircons range from 100 to $150 \mu \mathrm{m}$ (Figure 7). The $\mathrm{U}$ content ranges from 249.1 to $1094.1 \mathrm{ppm}$, Pb content ranges from 12.1 to $124 \mathrm{ppm}$, and Th content ranges from 132.1 to $1072 \mathrm{ppm}$. Th/U ratios are from 0.23 to 1.81 , and ${ }^{206} \mathrm{~Pb} /{ }^{238} \mathrm{U}$ ages are from $206.6 \pm 6.3$ to $232.9 \pm 7.1 \mathrm{Ma}$. The concordance age of the zircon grains is $230.8 \pm 1.6 \mathrm{Ma}(\mathrm{MSWD}=0.31$ ).

(3) G-3: Zircons are columnar crystals with grain sizes ranging from 50 to $150 \mu \mathrm{m}$, typical of acidic magmatic zircons, with $\mathrm{Th} / \mathrm{U}$ ratios of $0.12-2.07, \mathrm{~Pb}$ content from 17.9 to $265.71 \mathrm{ppm}$, Th content from 54.46 to $1425.03 \mathrm{ppm}$, and $U$ content from 295.74 to $12,287.53 \mathrm{ppm}$. The obtained ${ }^{206} \mathrm{~Pb} /{ }^{238} \mathrm{U}$ ages reveal two notably different groups: the first group is from $200.5 \pm 3.51$ to $203.9 \pm 3.55 \mathrm{Ma}$ with a concordance age of $203.1 \pm 1.6 \mathrm{Ma}$ (MSWD = 7.2). The ${ }^{206} \mathrm{~Pb} /{ }^{238} \mathrm{U}$ age of the second group ranges from $218.2 \pm 4.11$ to $226.8 \pm 4.05 \mathrm{Ma}$, and the concordance age is $224.8 \pm 1.6 \mathrm{Ma}$ (MSWD = 0.047) (Figure $8 \mathrm{~d}$ ).

(4) G-4: Zircons from granite porphyry have a minimum size ranging from 50 to $100 \mu \mathrm{m}$. The oscillating zones are not well developed. Three out of thirty analysis spots have $\mathrm{Pb}$ contents ranging from 17.6 to $21.6 \mathrm{ppm}$, Th contents from 268.1 to $555.5 \mathrm{ppm}$, and $\mathrm{U}$ contents from 495.9 to $810.2 \mathrm{ppm}$. The Th/U ratios range from 0.54 to 0.77 and obtained ${ }^{206} \mathrm{~Pb} /{ }^{238} \mathrm{U}$ ages range from $134.2 \pm 4.2$ to $137.5 \pm 4.2 \mathrm{Ma}$. The concordance age is $135.5 \pm 2.4 \mathrm{Ma}(\mathrm{MSWD}=1.3)$ (Figure $8 \mathrm{f})$. Twenty-one out of thirty analyses yield ${ }^{206} \mathrm{~Pb} /{ }^{238} \mathrm{U}$ ages from $202.4 \pm 6.1$ to $231.9 \pm 7.1 \mathrm{Ma}$, and the concordance age is $222.9 \pm 2.2 \mathrm{Ma}(\mathrm{MSWD}=0.13$ ) (Figure 8e), which is consistent with the wallrock two-mica monzogranite (G-2). In addition, the scattered points are 2586.5 Ma, 808.3 Ma, 1068 Ma, and $421 \mathrm{Ma}$, which may represent the formation ages of inheritable magmatic zircons or xenocrysts.

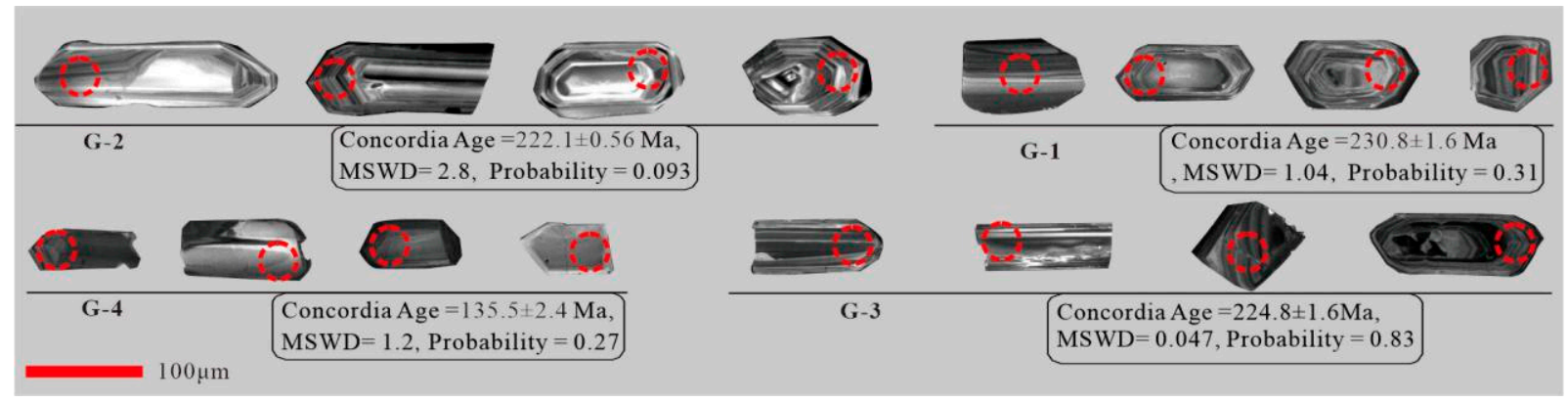

Figure 7. Analysis sites and cathodoluminescence (CL) images of typical zircons. 
Table 2. The LA-ICP-MS U-Pb analysis results of zircons from granitoids from Chuankou ore field.

\begin{tabular}{|c|c|c|c|c|c|c|c|c|c|c|c|c|c|c|c|c|c|}
\hline \multirow{3}{*}{ Lithology } & \multirow{3}{*}{ Spot } & \multicolumn{6}{|c|}{$\mathrm{W}_{\mathrm{B}} / \mathbf{1 0}^{-6}$} & \multicolumn{4}{|c|}{ Common Pb Isotope Ratio $( \pm 1 \sigma)$} & \multicolumn{6}{|c|}{ Common Pb Isotope Age $(\mathrm{Ma})( \pm \mathbf{1 \sigma})$} \\
\hline & & \multirow{2}{*}{$\mathrm{Pb}$} & \multirow{2}{*}{ Th } & \multirow{2}{*}{$\mathbf{U}$} & \multirow{2}{*}{$\mathrm{Th} / \mathrm{U}$} & \multicolumn{2}{|c|}{${ }^{207} \mathrm{~Pb} /{ }^{206} \mathrm{~Pb}$} & \multicolumn{2}{|c|}{${ }^{207} \mathrm{~Pb} /{ }^{235} \mathrm{U}$} & \multicolumn{2}{|c|}{${ }^{206} \mathrm{~Pb} /{ }^{238} \mathrm{U}$} & \multicolumn{2}{|c|}{${ }^{207} \mathrm{~Pb} /{ }^{206} \mathrm{~Pb}$} & \multicolumn{2}{|c|}{${ }^{207} \mathrm{~Pb} /{ }^{235} \mathrm{U}$} & \multicolumn{2}{|c|}{${ }^{206} \mathrm{~Pb} / 238 \mathrm{U}$} \\
\hline & & & & & & Ratio & $\pm \mathbf{1 \sigma}$ & Ratio & $\pm \mathbf{1 \sigma}$ & Ratio & $\pm \mathbf{1 \sigma}$ & Age & $\pm \mathbf{1 \sigma}$ & Age & $\pm \mathbf{1 \sigma}$ & Age & $\pm \mathbf{1 \sigma}$ \\
\hline \multirow{21}{*}{ G-2 } & HNCK2_1 & 111.67 & 634.03 & 822.29 & 0.77 & 0.05187 & 0.00107 & 0.2437 & 0.0052 & 0.03453 & 0.00036 & 279.6 & 46.38 & 221.4 & 4.25 & 218.9 & 2.23 \\
\hline & HNCK2_3 & 219.30 & 865.55 & 1673.73 & 0.52 & 0.08279 & 0.00138 & 0.36848 & 0.00643 & 0.03332 & 0.00034 & 1264.3 & 32.17 & 318.5 & 4.77 & 211.3 & 2.11 \\
\hline & HNCK2_4 & 138.38 & 338.58 & 964.59 & 0.35 & 0.05047 & 0.00085 & 0.2518 & 0.00436 & 0.03648 & 0.00035 & 216.5 & 38.5 & 228 & 3.54 & 231 & 2.19 \\
\hline & HNCK2_5 & 207.26 & 722.05 & 1456.89 & 0.50 & 0.05123 & 0.00072 & 0.25494 & 0.00363 & 0.03617 & 0.00033 & 251.1 & 32 & 230.6 & 2.93 & 229.1 & 2.08 \\
\hline & HNCK2_6 & 143.88 & 370.56 & 1028.90 & 0.36 & 0.05021 & 0.00076 & 0.24828 & 0.00383 & 0.03556 & 0.00033 & 204.7 & 34.81 & 225.2 & 3.12 & 225.2 & 2.08 \\
\hline & HNCK2_7 & 119.68 & 563.72 & 842.52 & 0.67 & 0.05163 & 0.00085 & 0.25473 & 0.00429 & 0.03612 & 0.00035 & 269.1 & 37.18 & 230.4 & 3.47 & 228.7 & 2.16 \\
\hline & HNCK2_99 & 166.85 & 528.06 & 1187.55 & 0.44 & 0.05304 & 0.00104 & 0.25186 & 0.00512 & 0.03572 & 0.00037 & 330.4 & 43.65 & 228.1 & 4.15 & 226.3 & 2.27 \\
\hline & HNCK2_10 & 102.06 & 716.95 & 760.66 & 0.94 & 0.05487 & 0.00263 & 0.24542 & 0.01247 & 0.03411 & 0.0006 & 407.1 & 103.55 & 222.9 & 10.16 & 216.2 & 3.72 \\
\hline & HNCK2_11 & 142.53 & 520.13 & 966.33 & 0.54 & 0.05188 & 0.00172 & 0.26223 & 0.00923 & 0.0375 & 0.0005 & 280 & 73.86 & 236.5 & 7.42 & 237.3 & 3.09 \\
\hline & HNCK2_11 & 142.53 & 520.13 & 966.33 & 0.54 & 0.05188 & 0.00172 & 0.26223 & 0.00923 & 0.0375 & 0.0005 & 280 & 73.86 & 236.5 & 7.42 & 237.3 & 3.09 \\
\hline & HNCK2_13 & 69.90 & 396.22 & 489.87 & 0.81 & 0.05279 & 0.00148 & 0.25912 & 0.00767 & 0.03627 & 0.00044 & 319.6 & 62.55 & 234 & 6.18 & 229.7 & 2.71 \\
\hline & HNCK2_14 & 84.02 & 538.83 & 598.67 & 0.90 & 0.0517 & 0.00101 & 0.25411 & 0.00515 & 0.03568 & 0.00036 & 272.3 & 44.27 & 229.9 & 4.17 & 226 & 2.25 \\
\hline & HNCK2_15 & 101.63 & 335.55 & 741.11 & 0.45 & 0.05071 & 0.00109 & 0.24708 & 0.00551 & 0.03486 & 0.00037 & 227.8 & 48.78 & 224.2 & 4.49 & 220.9 & 2.28 \\
\hline & HNCK2_16 & 343.28 & 558.99 & 2405.11 & 0.23 & 0.04871 & 0.0012 & 0.2509 & 0.00655 & 0.03628 & 0.0004 & 133.9 & 57.1 & 227.3 & 5.32 & 229.7 & 2.52 \\
\hline & HNCK2_17 & 149.38 & 840.17 & 1097.67 & 0.77 & 0.05217 & 0.00237 & 0.24238 & 0.01167 & 0.03459 & 0.00057 & 293.1 & 100.38 & 220.4 & 9.54 & 219.2 & 3.57 \\
\hline & HNCK2_18 & 154.13 & 482.91 & 1137.57 & 0.42 & 0.05263 & 0.00127 & 0.24363 & 0.00615 & 0.03444 & 0.00038 & 312.8 & 53.81 & 221.4 & 5.02 & 218.3 & 2.4 \\
\hline & HNCK2_21 & 67.82 & 212.21 & 506.25 & 0.42 & 0.04879 & 0.00315 & 0.23994 & 0.01648 & 0.03405 & 0.00073 & 137.8 & 145.22 & 218.4 & 13.49 & 215.9 & 4.54 \\
\hline & HNCK2_22 & 97.03 & 330.68 & 698.64 & 0.47 & 0.05049 & 0.00127 & 0.25045 & 0.00661 & 0.0353 & 0.0004 & 217.4 & 57.16 & 226.9 & 5.37 & 223.6 & 2.47 \\
\hline & HNCK2_23 & 120.40 & 382.15 & 873.04 & 0.44 & 0.0531 & 0.00246 & 0.24878 & 0.01227 & 0.03505 & 0.00059 & 333.2 & 101.69 & 225.6 & 9.98 & 222.1 & 3.69 \\
\hline & HNCK2_24 & 142.21 & 252.36 & 1031.23 & 0.24 & 0.05332 & 0.00206 & 0.23983 & 0.00979 & 0.03505 & 0.00052 & 342.5 & 84.94 & 218.3 & 8.02 & 222.1 & 3.23 \\
\hline & HNCK2_25 & 154.89 & 490.49 & 1134.71 & 0.43 & 0.05139 & 0.00174 & 0.24516 & 0.00881 & 0.03469 & 0.00047 & 258.3 & 76.15 & 222.6 & 7.19 & 219.8 & 2.91 \\
\hline \multirow{12}{*}{ G-3 } & HNCK10-10_1 & 42.76 & 600.88 & 698.24 & 0.86 & 0.05258 & 0.00127 & 0.25768 & 0.00564 & 0.03554 & 0.00064 & 310.5 & 54.15 & 232.8 & 4.56 & 225.1 & 4 \\
\hline & HNCK10-10_2 & 17.91 & 245.77 & 295.74 & 0.83 & 0.05309 & 0.00277 & 0.26111 & 0.01289 & 0.03567 & 0.00081 & 332.6 & 113.95 & 235.6 & 10.38 & 225.9 & 5.06 \\
\hline & HNCK10-10_3 & 243.65 & 1330.68 & 11437.47 & 0.12 & 0.05037 & 0.00113 & 0.24808 & 0.00499 & 0.03572 & 0.00064 & 211.9 & 51.39 & 225 & 4.06 & 226.2 & 3.97 \\
\hline & HNCK10-10_4 & 255.31 & 1425.03 & 12287.54 & 0.12 & 0.05024 & 0.00113 & 0.2469 & 0.00495 & 0.03564 & 0.00063 & 205.9 & 51.36 & 224.1 & 4.03 & 225.8 & 3.95 \\
\hline & HNCK10-10_5 & 60.08 & 691.19 & 1489.53 & 0.46 & 0.05393 & 0.00139 & 0.26633 & 0.00624 & 0.03581 & 0.00065 & 367.9 & 57.16 & 239.8 & 5 & 226.8 & 4.05 \\
\hline & HNCK10-10_6 & 26.78 & 350.62 & 302.75 & 1.16 & 0.05167 & 0.00153 & 0.25217 & 0.00687 & 0.03539 & 0.00066 & 270.7 & 66.56 & 228.3 & 5.57 & 224.2 & 4.11 \\
\hline & HNCK10-10_7 & 22.17 & 242.09 & 672.73 & 0.36 & 0.04949 & 0.00203 & 0.21926 & 0.00844 & 0.03213 & 0.00065 & 171 & 92.98 & 201.3 & 7.03 & 203.9 & 4.08 \\
\hline & HNCK10-10_8 & 43.42 & 616.93 & 658.15 & 0.94 & 0.05204 & 0.00149 & 0.25532 & 0.00666 & 0.03558 & 0.00066 & 287.2 & 63.89 & 230.9 & 5.39 & 225.4 & 4.09 \\
\hline & HNCK10-10_9 & 98.45 & 1376.99 & 1277.03 & 1.08 & 0.05904 & 0.00192 & 0.28027 & 0.00839 & 0.03442 & 0.00066 & 568.7 & 69.2 & 250.9 & 6.65 & 218.2 & 4.11 \\
\hline & HNCK10-10_10 & 39.09 & 564.16 & 453.26 & 1.24 & 0.05081 & 0.00143 & 0.24846 & 0.00636 & 0.03546 & 0.00065 & 232.4 & 63.56 & 225.3 & 5.17 & 224.6 & 4.05 \\
\hline & HNCK10-10_11 & 69.24 & 1035.35 & 969.17 & 1.07 & 0.06305 & 0.00182 & 0.30768 & 0.00806 & 0.03539 & 0.00066 & 709.8 & 60.22 & 272.4 & 6.26 & 224.2 & 4.08 \\
\hline & HNCK10-10_12 & 127.14 & 1253.68 & 3790.46 & 0.33 & 0.0511 & 0.00116 & 0.25108 & 0.00503 & 0.03563 & 0.00063 & 245.3 & 51.65 & 227.4 & 4.08 & 225.7 & 3.9 \\
\hline
\end{tabular}


Table 2. Cont.

\begin{tabular}{|c|c|c|c|c|c|c|c|c|c|c|c|c|c|c|c|c|c|}
\hline \multirow{3}{*}{ Lithology } & \multirow{3}{*}{ Spot } & \multicolumn{6}{|c|}{$\mathrm{W}_{\mathrm{B}} / 10^{-6}$} & \multicolumn{4}{|c|}{ Common $\mathrm{Pb}$ Isotope Ratio $( \pm 1 \sigma)$} & \multicolumn{6}{|c|}{ Common Pb Isotope Age (Ma) $( \pm 1 \sigma)$} \\
\hline & & \multirow{2}{*}{$\mathbf{P b}$} & \multirow{2}{*}{ Th } & \multirow{2}{*}{$\mathbf{U}$} & \multirow{2}{*}{$\mathrm{Th} / \mathrm{U}$} & \multicolumn{2}{|c|}{${ }^{207} \mathrm{~Pb} /{ }^{206} \mathrm{~Pb}$} & \multicolumn{2}{|c|}{${ }^{207} \mathrm{~Pb} /{ }^{235} \mathrm{U}$} & \multicolumn{2}{|c|}{${ }^{206} \mathrm{~Pb} /{ }^{238} \mathrm{U}$} & \multicolumn{2}{|c|}{${ }^{207} \mathrm{~Pb} /{ }^{206} \mathrm{~Pb}$} & \multicolumn{2}{|c|}{${ }^{207} \mathrm{~Pb} /{ }^{235} \mathrm{U}$} & \multicolumn{2}{|c|}{${ }^{206} \mathrm{~Pb} /{ }^{238} \mathrm{U}$} \\
\hline & & & & & & Ratio & $\pm 1 \sigma$ & Ratio & $\pm \mathbf{1 \sigma}$ & Ratio & $\pm \mathbf{1 \sigma}$ & Age & $\pm \mathbf{1 \sigma}$ & Age & $\pm 1 \sigma$ & Age & $\pm \mathbf{1 \sigma}$ \\
\hline & HNCK10-10_13 & 56.35 & 534.15 & 2092.28 & 0.26 & 0.05136 & 0.00122 & 0.22757 & 0.0048 & 0.03213 & 0.00057 & 256.9 & 53.89 & 208.2 & 3.97 & 203.9 & 3.55 \\
\hline & HNCK10-10_15 & 57.28 & 516.40 & 1802.74 & 0.29 & 0.05083 & 0.0012 & 0.22373 & 0.00465 & 0.03192 & 0.00056 & 233 & 53.57 & 205 & 3.86 & 202.6 & 3.51 \\
\hline & HNCK10-10_16 & 78.82 & 822.01 & 1698.14 & 0.48 & 0.05152 & 0.00145 & 0.22742 & 0.00579 & 0.03201 & 0.00058 & 264.3 & 63.34 & 208.1 & 4.79 & 203.1 & 3.63 \\
\hline & HNCK10-10_17 & 33.63 & 318.40 & 842.75 & 0.38 & 0.05267 & 0.00212 & 0.25369 & 0.00953 & 0.03494 & 0.0007 & 314.4 & 89.21 & 229.6 & 7.72 & 221.4 & 4.37 \\
\hline & HNCK10-10_18 & 91.12 & 1238.66 & 597.63 & 2.07 & 0.14575 & 0.00463 & 0.78474 & 0.02203 & 0.03905 & 0.00079 & 2296.6 & 53.66 & 588.2 & 12.53 & 246.9 & 4.91 \\
\hline & HNCK10-10_19 & 92.73 & 1071.44 & 1094.50 & 0.98 & 0.07741 & 0.00195 & 0.34175 & 0.00763 & 0.03202 & 0.00057 & 1131.7 & 49.48 & 298.5 & 5.77 & 203.2 & 3.57 \\
\hline & HNCK10-10_21 & 76.24 & 637.11 & 1117.42 & 0.57 & 0.0975 & 0.00224 & 0.4807 & 0.00957 & 0.03576 & 0.00062 & 1576.9 & 42.46 & 398.6 & 6.56 & 226.5 & 3.86 \\
\hline & HNCK10-10_22 & 271.11 & 428.32 & 416.13 & 1.03 & 0.50991 & 0.01159 & 5.83255 & 0.11417 & 0.08297 & 0.00144 & 4270.1 & 33.05 & 1951.3 & 16.97 & 513.8 & 8.55 \\
\hline & HNCK10-10_23 & 83.12 & 987.53 & 897.08 & 1.10 & 0.10308 & 0.00247 & 0.50779 & 0.01058 & 0.03574 & 0.00063 & 1680.3 & 43.69 & 417 & 7.12 & 226.3 & 3.9 \\
\hline & HNCK10-10_24 & 125.72 & 1038.20 & 1054.02 & 0.98 & 0.14594 & 0.00345 & 0.70767 & 0.01445 & 0.03518 & 0.00062 & 2298.9 & 40.12 & 543.4 & 8.59 & 222.9 & 3.83 \\
\hline & HNCK10-10_25 & 104.20 & 252.93 & 965.27 & 0.26 & 0.19257 & 0.00486 & 0.94152 & 0.02045 & 0.03547 & 0.00064 & 2764.2 & 40.83 & 673.7 & 10.7 & 224.7 & 4 \\
\hline & HNCK10-10_26 & 265.71 & 375.74 & 1953.79 & 0.19 & 0.18661 & 0.00425 & 1.23452 & 0.02408 & 0.04799 & 0.00082 & 2712.5 & 37.03 & 816.4 & 10.94 & 302.2 & 5.07 \\
\hline & HNCK10-10_27 & 36.95 & 54.46 & 429.53 & 0.13 & 0.09421 & 0.00217 & 0.94217 & 0.01861 & 0.07255 & 0.00125 & 1512.3 & 42.82 & 674 & 9.73 & 451.5 & 7.5 \\
\hline \multirow{21}{*}{ G-4 } & HNCK10-8_1 & 21.6 & 555.5 & 721.1 & 0.77 & 0.0502 & 0.0017 & 0.1461 & 0.0058 & 0.0211 & 0.0007 & 205.3 & 74.8 & 138.5 & 5.2 & 134.6 & 4.2 \\
\hline & HNCK10-8_2 & 19.1 & 209.9 & 401.4 & 0.52 & 0.0510 & 0.0018 & 0.2482 & 0.0104 & 0.0353 & 0.0011 & 241.3 & 80.1 & 225.1 & 8.5 & 223.6 & 6.9 \\
\hline & HNCK10-8_3 & 28.2 & 352.4 & 528.8 & 0.67 & 0.0508 & 0.0025 & 0.2392 & 0.0125 & 0.0341 & 0.0011 & 232.4 & 107.7 & 217.8 & 10.2 & 216.4 & 7.1 \\
\hline & HNCK10-8_5 & 75.9 & 929.0 & 1278.2 & 0.73 & 0.0522 & 0.0013 & 0.2501 & 0.0084 & 0.0347 & 0.0011 & 295.1 & 54.1 & 226.7 & 6.8 & 220.1 & 6.6 \\
\hline & HNCK10-8_6 & 33.0 & 356.7 & 653.3 & 0.55 & 0.0521 & 0.0013 & 0.2509 & 0.0087 & 0.0349 & 0.0011 & 289.8 & 57.6 & 227.3 & 7.0 & 221.3 & 6.7 \\
\hline & HNCK10-8_7 & 31.9 & 430.2 & 773.8 & 0.56 & 0.0520 & 0.0013 & 0.2512 & 0.0087 & 0.0350 & 0.0011 & 285.2 & 57.8 & 227.5 & 7.0 & 222.0 & 6.7 \\
\hline & HNCK10-8_8 & 10.9 & 155.8 & 112.5 & 1.39 & 0.0511 & 0.0023 & 0.2493 & 0.0124 & 0.0354 & 0.0012 & 245.3 & 100.8 & 226.0 & 10.1 & 224.1 & 7.2 \\
\hline & HNCK10-8_9 & 101.3 & 52.7 & 159.4 & 0.33 & 0.1793 & 0.0038 & 12.2054 & 0.3856 & 0.4937 & 0.0151 & 2646.4 & 35.1 & 2620.3 & 29.7 & 2586.5 & 65.1 \\
\hline & HNCK10-8_10 & 21.3 & 211.5 & 537.6 & 0.39 & 0.0507 & 0.0013 & 0.2469 & 0.0085 & 0.0353 & 0.0011 & 226.6 & 58.3 & 224.1 & 6.9 & 223.8 & 6.8 \\
\hline & HNCK10-8_11 & 27.2 & 414.5 & 424.7 & 0.98 & 0.0503 & 0.0016 & 0.2437 & 0.0095 & 0.0351 & 0.0011 & 210.2 & 72.8 & 221.4 & 7.8 & 222.5 & 6.8 \\
\hline & HNCK10-8_12 & 79.9 & 814.2 & 2356.1 & 0.35 & 0.0506 & 0.0011 & 0.2451 & 0.0079 & 0.0351 & 0.0011 & 223.3 & 51.1 & 222.6 & 6.4 & 222.5 & 6.7 \\
\hline & HNCK10-8_13 & 17.6 & 268.1 & 495.9 & 0.54 & 0.0497 & 0.0018 & 0.1478 & 0.0063 & 0.0216 & 0.0007 & 182.0 & 82.8 & 140.0 & 5.6 & 137.5 & 4.2 \\
\hline & HNCK10-8_14 & 25.7 & 314.2 & 660.4 & 0.48 & 0.0503 & 0.0015 & 0.2494 & 0.0092 & 0.0360 & 0.0011 & 209.2 & 66.3 & 226.1 & 7.5 & 227.7 & 6.9 \\
\hline & HNCK10-8_15 & 12.7 & 138.5 & 371.7 & 0.37 & 0.0517 & 0.0021 & 0.2395 & 0.0108 & 0.0336 & 0.0011 & 269.8 & 89.1 & 218.0 & 8.9 & 213.2 & 6.7 \\
\hline & HNCK10-8_16 & 17.6 & 454.9 & 810.2 & 0.56 & 0.0486 & 0.0019 & 0.1411 & 0.0064 & 0.0210 & 0.0007 & 129.6 & 90.4 & 134.0 & 5.7 & 134.2 & 4.2 \\
\hline & HNCK10-8_17 & 25.7 & 341.6 & 431.3 & 0.79 & 0.0522 & 0.0024 & 0.2556 & 0.0128 & 0.0355 & 0.0012 & 294.5 & 101.8 & 231.1 & 10.4 & 224.9 & 7.2 \\
\hline & HNCK10-8_18 & 28.7 & 476.5 & 659.4 & 0.72 & 0.0522 & 0.0021 & 0.1786 & 0.0080 & 0.0248 & 0.0008 & 293.9 & 87.4 & 166.8 & 6.9 & 158.0 & 5.0 \\
\hline & HNCK10-8_19 & 21.5 & 301.6 & 311.5 & 0.97 & 0.0511 & 0.0020 & 0.2391 & 0.0108 & 0.0340 & 0.0011 & 243.6 & 89.6 & 217.7 & 8.9 & 215.3 & 6.7 \\
\hline & HNCK10-8_20 & 32.5 & 356.3 & 803.7 & 0.44 & 0.0509 & 0.0013 & 0.2453 & 0.0083 & 0.0350 & 0.0011 & 234.0 & 57.1 & 222.8 & 6.8 & 221.7 & 6.7 \\
\hline & HNCK10-8_22 & 28.8 & 477.0 & 748.4 & 0.64 & 0.0510 & 0.0019 & 0.1998 & 0.0085 & 0.0284 & 0.0009 & 238.6 & 82.4 & 185.0 & 7.2 & 180.8 & 5.6 \\
\hline & HNCK10-8_22 & 50.1 & 114.4 & 407.6 & 0.28 & 0.0667 & 0.0019 & 1.2282 & 0.0442 & 0.1336 & 0.0041 & 827.5 & 57.8 & 813.5 & 20.1 & 808.3 & 23.5 \\
\hline
\end{tabular}


Table 2. Cont.

\begin{tabular}{|c|c|c|c|c|c|c|c|c|c|c|c|c|c|c|c|c|c|}
\hline \multirow{3}{*}{ Lithology } & \multirow{3}{*}{ Spot } & \multicolumn{6}{|c|}{$\mathrm{W}_{\mathrm{B}} / 10^{-6}$} & \multicolumn{4}{|c|}{ Common $\mathrm{Pb}$ Isotope Ratio $( \pm 1 \sigma)$} & \multicolumn{6}{|c|}{ Common Pb Isotope Age (Ma) $( \pm 1 \sigma)$} \\
\hline & & \multirow{2}{*}{$\mathbf{P b}$} & \multirow{2}{*}{ Th } & \multirow{2}{*}{$\mathbf{U}$} & \multirow{2}{*}{$\mathrm{Th} / \mathrm{U}$} & \multicolumn{2}{|c|}{${ }^{207} \mathrm{~Pb} /{ }^{206} \mathrm{~Pb}$} & \multicolumn{2}{|c|}{${ }^{207} \mathrm{~Pb} /{ }^{235} \mathrm{U}$} & \multicolumn{2}{|c|}{${ }^{206} \mathrm{~Pb} /{ }^{238} \mathrm{U}$} & \multicolumn{2}{|c|}{${ }^{207} \mathrm{~Pb} /{ }^{206} \mathrm{~Pb}$} & \multicolumn{2}{|c|}{${ }^{207} \mathrm{~Pb} /{ }^{235} \mathrm{U}$} & \multicolumn{2}{|c|}{${ }^{206} \mathrm{~Pb} /{ }^{238} \mathrm{U}$} \\
\hline & & & & & & Ratio & $\pm \mathbf{1 \sigma}$ & Ratio & $\pm \mathbf{1 \sigma}$ & Ratio & $\pm \mathbf{1 \sigma}$ & Age & $\pm \mathbf{1 \sigma}$ & Age & $\pm \mathbf{1 \sigma}$ & Age & $\pm \mathbf{1 \sigma}$ \\
\hline & HNCK10-8_23 & 35.8 & 393.7 & 717.2 & 0.55 & 0.0523 & 0.0013 & 0.2517 & 0.0084 & 0.0349 & 0.0011 & 299.2 & 54.9 & 228.0 & 6.8 & 221.1 & 6.6 \\
\hline & HNCK10-8_25 & 59.8 & 811.2 & 825.3 & 0.98 & 0.0498 & 0.0016 & 0.2412 & 0.0093 & 0.0352 & 0.0011 & 184.0 & 71.6 & 219.4 & 7.6 & 222.7 & 6.8 \\
\hline & HNCK10-8_26 & 26.6 & 280.5 & 770.5 & 0.36 & 0.0498 & 0.0015 & 0.2191 & 0.0082 & 0.0319 & 0.0010 & 186.8 & 67.9 & 201.2 & 6.8 & 202.4 & 6.1 \\
\hline & HNCK10-8_27 & 46.4 & 635.0 & 472.6 & 1.34 & 0.0506 & 0.0017 & 0.2454 & 0.0097 & 0.0352 & 0.0011 & 220.7 & 74.0 & 222.9 & 7.9 & 223.0 & 6.8 \\
\hline & HNCK10-8_28 & 18.5 & 265.8 & 264.0 & 1.01 & 0.0516 & 0.0026 & 0.2493 & 0.0133 & 0.0350 & 0.0012 & 268.9 & 110.4 & 226.0 & 10.8 & 221.8 & 7.1 \\
\hline & HNCK10-8_29 & 40.7 & 57.5 & 252.5 & 0.23 & 0.0742 & 0.0022 & 1.8441 & 0.0674 & 0.1802 & 0.0056 & 1047.2 & 57.7 & 1061.3 & 24.1 & 1068.0 & 30.6 \\
\hline \multirow{19}{*}{ G-1 } & HNCK10-14_1 & 27.8 & 346.2 & 335.6 & 1.03 & 0.0516 & 0.0016 & 0.2591 & 0.0097 & 0.0364 & 0.0011 & 268.6 & 67.3 & 234.0 & 7.8 & 230.5 & 7.0 \\
\hline & HNCK10-14_2 & 22.3 & 253.8 & 411.7 & 0.62 & 0.0513 & 0.0016 & 0.2581 & 0.0098 & 0.0365 & 0.0011 & 252.1 & 69.5 & 233.1 & 7.9 & 231.2 & 7.0 \\
\hline & HNCK10-14_3 & 16.2 & 169.1 & 378.6 & 0.45 & 0.0499 & 0.0017 & 0.2531 & 0.0102 & 0.0368 & 0.0011 & 190.1 & 76.9 & 229.1 & 8.3 & 232.9 & 7.1 \\
\hline & HNCK10-14_4 & 12.1 & 132.1 & 249.1 & 0.53 & 0.0516 & 0.0018 & 0.2587 & 0.0105 & 0.0364 & 0.0011 & 267.5 & 76.7 & 233.6 & 8.5 & 230.2 & 7.0 \\
\hline & HNCK10-14_5 & 49.0 & 263.9 & 639.9 & 0.41 & 0.0553 & 0.0013 & 0.5142 & 0.0168 & 0.0675 & 0.0020 & 422.4 & 51.2 & 421.3 & 11.3 & 421.0 & 12.3 \\
\hline & HNCK10-14_6 & 45.9 & 608.4 & 346.4 & 1.76 & 0.0526 & 0.0052 & 0.2628 & 0.0255 & 0.0362 & 0.0015 & 310.4 & 209.4 & 236.9 & 20.5 & 229.5 & 9.3 \\
\hline & HNCK10-14_7 & 15.9 & 182.4 & 288.9 & 0.63 & 0.0508 & 0.0016 & 0.2548 & 0.0097 & 0.0363 & 0.0011 & 233.6 & 70.7 & 230.5 & 7.9 & 230.1 & 7.0 \\
\hline & HNCK10-14_8 & 22.6 & 244.3 & 549.7 & 0.44 & 0.0507 & 0.0015 & 0.2555 & 0.0095 & 0.0365 & 0.0011 & 227.5 & 68.1 & 231.1 & 7.7 & 231.4 & 7.0 \\
\hline & HNCK10-14_9 & 15.8 & 153.7 & 396.2 & 0.39 & 0.0520 & 0.0019 & 0.2587 & 0.0109 & 0.0361 & 0.0011 & 284.9 & 80.7 & 233.6 & 8.8 & 228.5 & 7.0 \\
\hline & HNCK10-14_10 & 67.0 & 970.4 & 766.9 & 1.27 & 0.0512 & 0.0016 & 0.2562 & 0.0097 & 0.0363 & 0.0011 & 247.6 & 69.1 & 231.6 & 7.8 & 230.0 & 6.9 \\
\hline & HNCK10-14_12 & 23.6 & 311.3 & 300.2 & 1.04 & 0.0518 & 0.0021 & 0.2584 & 0.0117 & 0.0362 & 0.0011 & 276.4 & 89.1 & 233.4 & 9.4 & 229.1 & 7.1 \\
\hline & HNCK10-14_13 & 27.6 & 278.7 & 648.5 & 0.43 & 0.0514 & 0.0015 & 0.2592 & 0.0093 & 0.0366 & 0.0011 & 259.1 & 64.0 & 234.0 & 7.5 & 231.5 & 6.9 \\
\hline & HNCK10-14_14 & 124.0 & 1694.3 & 1462.9 & 1.16 & 0.0509 & 0.0017 & 0.2284 & 0.0091 & 0.0326 & 0.0010 & 234.2 & 75.5 & 208.9 & 7.5 & 206.6 & 6.3 \\
\hline & HNCK10-14_15 & 25.8 & 250.6 & 667.0 & 0.38 & 0.0519 & 0.0014 & 0.2603 & 0.0090 & 0.0364 & 0.0011 & 280.8 & 59.4 & 234.9 & 7.3 & 230.3 & 6.8 \\
\hline & HNCK10-14_16 & 38.5 & 364.2 & 943.7 & 0.39 & 0.0507 & 0.0012 & 0.2549 & 0.0083 & 0.0365 & 0.0011 & 227.5 & 53.7 & 230.6 & 6.7 & 230.8 & 6.8 \\
\hline & HNCK10-14_17 & 29.9 & 219.9 & 944.7 & 0.23 & 0.0519 & 0.0014 & 0.2599 & 0.0090 & 0.0363 & 0.0011 & 279.5 & 59.6 & 234.6 & 7.3 & 230.1 & 6.8 \\
\hline & HNCK10-14_18 & 30.3 & 290.7 & 676.2 & 0.43 & 0.0514 & 0.0023 & 0.2589 & 0.0127 & 0.0365 & 0.0012 & 258.5 & 99.7 & 233.8 & 10.2 & 231.3 & 7.3 \\
\hline & HNCK10-14_19 & 38.6 & 355.7 & 1094.1 & 0.33 & 0.0497 & 0.0012 & 0.2514 & 0.0082 & 0.0367 & 0.0011 & 179.3 & 53.8 & 227.7 & 6.6 & 232.4 & 6.9 \\
\hline & HNCK10-14_20 & 22.7 & 231.7 & 502.5 & 0.46 & 0.0516 & 0.0015 & 0.2603 & 0.0093 & 0.0366 & 0.0011 & 268.9 & 63.3 & 234.9 & 7.5 & 231.5 & 6.9 \\
\hline
\end{tabular}



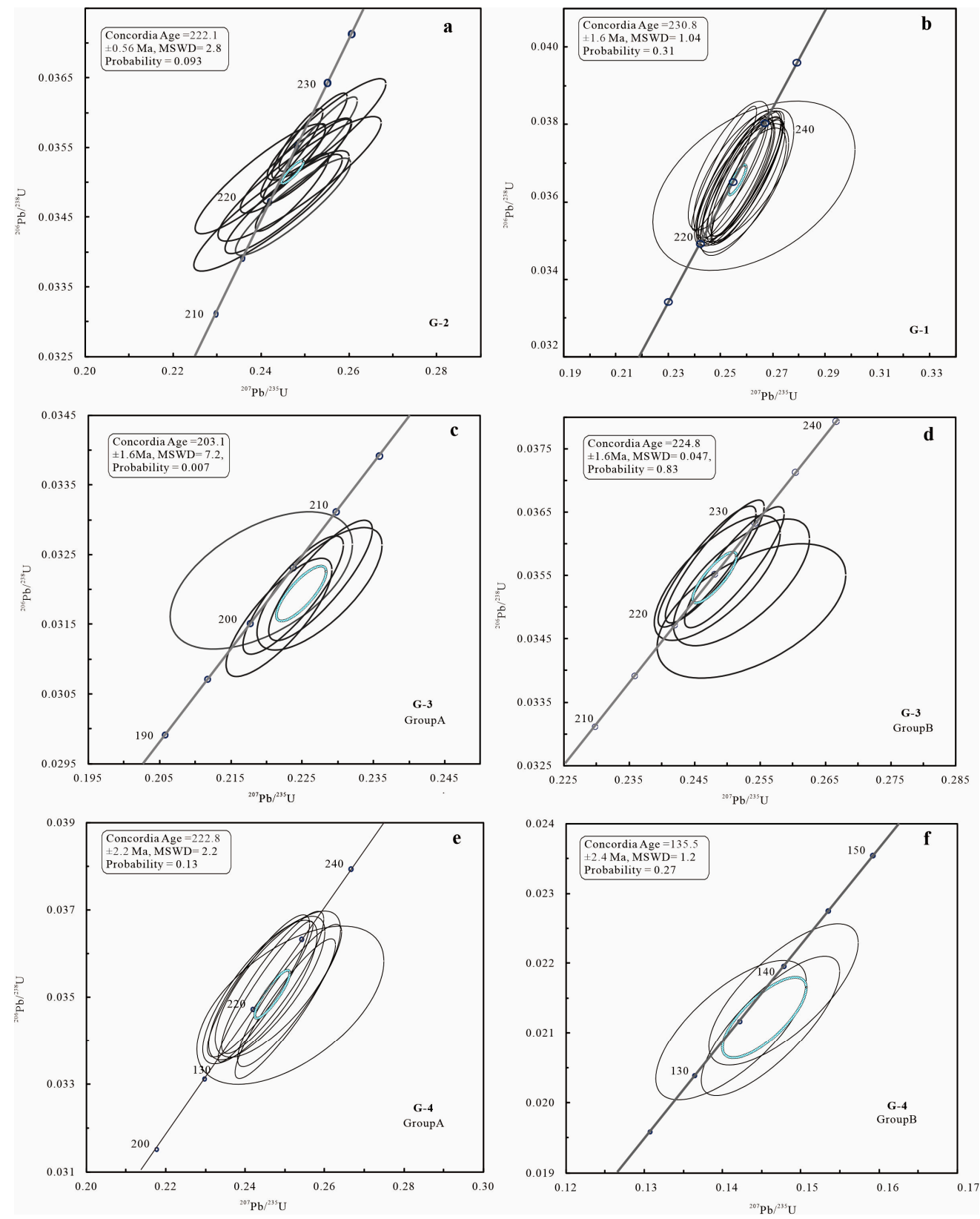

Figure 8. The zircon concordance diagrams of granitoids from Chuankou ore field. (a) G-2; (b) G-1; (c,d) G-3, (e,f) G-4.

\subsection{Geochemistry}

Thirteen samples from the Chuankou ore field were analyzed and the analysis results are listed in Table 3. 
Table 3. The geochemical analysis results of samples of granitoids from Chuankou ore field.

\begin{tabular}{|c|c|c|c|c|c|c|c|c|c|c|c|c|c|}
\hline Sample & HNCK10-1 & HNCK10-3 & HNCK1 & HNCK2 & HNCK10-14-1 & HNCK10-14 & HNCK10-6 & HNCK3 & HNCK10-10 & HNCK10-9 & HNCK10-5 & HNCK10-8 & HNOH1 \\
\hline Lithology & \multicolumn{4}{|c|}{ Greisenization G-2 } & \multicolumn{2}{|c|}{ G-1 } & \multicolumn{2}{|c|}{ G-2 } & \multicolumn{3}{|c|}{ G-3 } & \multicolumn{2}{|c|}{ G-4 } \\
\hline \multicolumn{14}{|c|}{ wt. $\%$} \\
\hline $\mathrm{SiO}_{2}$ & 83.64 & 84.07 & 82.53 & 84.65 & 76.86 & 76.79 & 76.08 & 78.36 & 77.36 & 74.47 & 77.61 & 75.52 & 75.53 \\
\hline $\mathrm{Al}_{2} \mathrm{O}_{3}$ & 9.81 & 9.56 & 9.99 & 9.32 & 12.26 & 12.37 & 12.72 & 13.74 & 13.54 & 16.47 & 12.91 & 14.77 & 14.77 \\
\hline $\mathrm{Fe}_{2} \mathrm{O}_{3}$ & 0.24 & 0.29 & 1.82 & 0.94 & 0.04 & 0.08 & 0.1 & 1.14 & 0.28 & 0.63 & 0.28 & 0.22 & 0.23 \\
\hline $\mathrm{FeO}$ & 0.68 & 0.73 & 1.13 & 0.6 & 1.41 & 1.36 & 1.12 & 0.76 & 0.39 & 0.38 & 0.68 & 0.63 & 0.63 \\
\hline $\mathrm{MnO}$ & 0.07 & 0.23 & 0.12 & 0.07 & 0.09 & 0.09 & 0.1 & 0.13 & 0.04 & 0.04 & 0.26 & 0.04 & 0.04 \\
\hline $\mathrm{MgO}$ & 0.15 & 0.14 & 0.24 & 0.38 & 0.34 & 0.35 & 0.26 & 0.33 & 0.07 & 0.07 & 0.27 & 0.35 & 0.33 \\
\hline $\mathrm{Na}_{2} \mathrm{O}$ & 2.27 & 0.07 & 0.01 & 0.01 & 3.07 & 3.08 & 3.17 & 0.01 & 3.54 & 0.14 & 0.12 & 0.08 & 0.08 \\
\hline $\mathrm{K}_{2} \mathrm{O}$ & 1.72 & 2.71 & 2.62 & 1.52 & 4 & 3.98 & 4.27 & 3.5 & 3.37 & 3.38 & 3.44 & 4.77 & 4.79 \\
\hline $\mathrm{P}_{2} \mathrm{O}_{5}$ & 0.06 & 0.04 & 0.01 & 0.01 & 0.06 & 0.06 & 0.06 & 0.07 & 0.02 & 0.02 & 0.04 & 0.04 & 0.04 \\
\hline LOI & 1.1 & 1.66 & 1.66 & 2.26 & 0.61 & 0.52 & 0.82 & 2.64 & 0.86 & 4.09 & 3.51 & 2.79 & 2.74 \\
\hline SUM & 99.93 & 99.78 & 100.23 & 99.85 & 99.86 & 99.77 & 99.52 & 100.88 & 99.66 & 99.84 & 99.84 & 99.61 & 99.6 \\
\hline \multicolumn{14}{|c|}{ ppm } \\
\hline $\mathrm{Ni}$ & 1.48 & 1.24 & 2.06 & 1.73 & 4 & 3.72 & 2.87 & 1.78 & 1.32 & 27.58 & 1.6 & 2.03 & 2.31 \\
\hline $\mathrm{Pb}$ & 6.57 & 17.02 & 13.3 & 17.1 & 45.37 & 43.63 & 48.84 & 23.7 & 29.74 & 20.4 & 25.68 & 51.82 & 52.49 \\
\hline $\mathrm{Rb}$ & 508.82 & 292.23 & 469 & 236 & 394.43 & 395.14 & 479.56 & 676 & 791.82 & 744.72 & 682.86 & 453.74 & 452.3 \\
\hline $\mathrm{Sr}$ & 4.02 & 8.03 & 55 & 50.4 & 49.56 & 49.91 & 33.62 & 19.6 & 10.91 & 14.16 & 23.87 & 34.37 & 34.63 \\
\hline Ta & 11.19 & 4.18 & 6.96 & 4.03 & 5.47 & 5.17 & 4.03 & 8.7 & 30.55 & 48.27 & 33.48 & 3.27 & 2.93 \\
\hline Th & 6.64 & 7.65 & 6.78 & 6.23 & 18.35 & 17.53 & 14.94 & 14.8 & 5.63 & 6.6 & 6.65 & 31.72 & 32.33 \\
\hline $\mathrm{Tl}$ & 2.26 & 1.22 & 1.92 & 1.2 & 2.41 & 2.28 & 2.75 & 2.78 & 3.7 & 3.01 & 3.34 & 2.52 & 2.49 \\
\hline $\mathrm{U}$ & 23.8 & 30.92 & 3.2 & 1.86 & 20.63 & 19.99 & 20.03 & 44.6 & 12.76 & 3.71 & 23.37 & 9.06 & 9.03 \\
\hline $\mathrm{V}$ & 3.93 & 5.66 & 4.98 & 3.03 & 13.89 & 13.57 & 8 & 1.64 & 3.25 & 3.59 & 3.19 & 13.15 & 13.36 \\
\hline $\mathrm{W}$ & 7.62 & 38.2 & 60.3 & 25 & 2.81 & 2.09 & 4.34 & 12.2 & 11.52 & 11.97 & 9.59 & 7.31 & 7.79 \\
\hline $\mathrm{Zn}$ & 21.38 & 51.21 & 28.7 & 13.7 & 37.82 & 38.2 & 34.68 & 26.2 & 19.92 & 27.1 & 32.87 & 11.96 & 14.86 \\
\hline $\mathrm{Zr}$ & 24.61 & 43.07 & 28.9 & 27.9 & 79.95 & 78.01 & 63.17 & 41.4 & 21.25 & 24.04 & 23.23 & 150.11 & 147.78 \\
\hline $\mathrm{La}$ & 2.4 & 5.32 & 2.62 & 5.5 & 24.76 & 22.63 & 15.3 & 3.49 & 16.18 & 7.09 & 4.29 & 47.67 & 50.33 \\
\hline $\mathrm{Ce}$ & 6.99 & 11.77 & 5.72 & 8.92 & 51.33 & 47.85 & 34.46 & 10.7 & 36.15 & 6.38 & 14.31 & 94.54 & 100 \\
\hline $\operatorname{Pr}$ & 1.12 & 1.63 & 0.77 & 1.31 & 6.8 & 6.34 & 4.61 & 1.4 & 5.08 & 2.04 & 2.56 & 13.52 & 14.43 \\
\hline
\end{tabular}


Table 3. Cont.

\begin{tabular}{|c|c|c|c|c|c|c|c|c|c|c|c|c|c|}
\hline Sample & HNCK10-1 & HNCK10-3 & HNCK1 & HNCK2 & HNCK10-14-1 & HNCK10-14 & HNCK10-6 & HNCK3 & HNCK10-10 & HNCK10-9 & HNCK10-5 & HNCK10-8 & HNOH1 \\
\hline Lithology & \multicolumn{4}{|c|}{ Greisenization G-2 } & \multicolumn{2}{|c|}{ G-1 } & \multicolumn{2}{|c|}{ G-2 } & \multicolumn{3}{|c|}{ G-3 } & \multicolumn{2}{|c|}{ G-4 } \\
\hline $\mathrm{Nd}$ & 3.59 & 5.27 & 2.77 & 4.39 & 22.72 & 20.82 & 15.35 & 5.13 & 17.54 & 6.65 & 9.44 & 46 & 49.82 \\
\hline $\mathrm{Sm}$ & 1.5 & 1.6 & 0.9 & 1.22 & 4.9 & 4.62 & 3.86 & 2.14 & 6.44 & 1.98 & 4.25 & 8.95 & 9.82 \\
\hline $\mathrm{Eu}$ & 0.03 & 0.07 & 0 & 0.09 & 0.42 & 0.43 & 0.27 & 0.05 & 0.24 & 0.24 & 0.02 & 0.98 & 1.03 \\
\hline $\mathrm{Gd}$ & 1.13 & 1.28 & 0.89 & 1.14 & 4.01 & 3.98 & 3.34 & 1.75 & 3.94 & 1.13 & 2.61 & 6.41 & 6.96 \\
\hline $\mathrm{Tb}$ & 0.4 & 0.39 & 0.23 & 0.26 & 1 & 0.99 & 0.96 & 0.42 & 1.08 & 0.27 & 0.79 & 1.16 & 1.23 \\
\hline Dy & 2.63 & 2.49 & 1.64 & 1.81 & 6.23 & 6.31 & 6.32 & 2.63 & 5.6 & 1.25 & 4.23 & 5.02 & 5.31 \\
\hline Ho & 0.45 & 0.49 & 0.32 & 0.34 & 1.35 & 1.33 & 1.37 & 0.46 & 0.91 & 0.17 & 0.69 & 0.78 & 0.83 \\
\hline $\mathrm{Er}$ & 1.45 & 1.62 & 1.09 & 1.14 & 4.35 & 4.29 & 4.34 & 1.44 & 2.7 & 0.46 & 2.14 & 2.08 & 2.17 \\
\hline $\mathrm{Yb}$ & 2.86 & 2.75 & 1.74 & 1.69 & 4.25 & 4.14 & 4.6 & 2.7 & 4.5 & 0.75 & 3.25 & 1.8 & 1.91 \\
\hline $\mathrm{Lu}$ & 0.46 & 0.45 & 0.28 & 0.25 & 0.84 & 0.83 & 0.87 & 0.4 & 0.72 & 0.12 & 0.59 & 0.28 & 0.29 \\
\hline $\mathrm{Y}$ & 13.92 & 13.66 & 9.15 & 10.5 & 37.82 & 37.18 & 38.22 & 14 & 29.73 & 5 & 22.61 & 19.29 & 19.95 \\
\hline
\end{tabular}


G-1 is characterized by high $\mathrm{SiO}_{2}$ (76.79-76.86 wt.\%), ALK (7.06-7.07 wt.\%), Fe $\left(\mathrm{FeO}^{\mathrm{T}}=1.43-1.44\right.$ wt.\%), $\mathrm{Al}(\mathrm{A} / \mathrm{CNK}=1.11-1.113, \mathrm{~A} / \mathrm{NK}=1.307-1.32)$, and $\mathrm{K} / \mathrm{Na}$ ratios. G-2 has higher contents of $\mathrm{SiO}_{2}$ (76.08-78.36 wt.\%), $\mathrm{K}_{2} \mathrm{O}$ (3.50-4.27 wt.\%), and $\mathrm{Al}$ $(\mathrm{A} / \mathrm{CNK}=1.139-3.335)$ than $\mathrm{G}-1$. G-3 contains various contents of $\mathrm{SiO}_{2}(74.47-77.61 \%)$, $\mathrm{Al}_{2} \mathrm{O}_{3}$ (12.91-16.47 wt.\%) and characterized by low $\mathrm{Na}_{2} \mathrm{O}(0.12-3.54$ wt.\%) and ALK (3.52-6.91 wt. \%) contents. G-4 has the lowest $\mathrm{Na}_{2} \mathrm{O}$ content (0.08 wt.\%), MnO content (0.04 wt.\%), and has the highest $\mathrm{K}_{2} \mathrm{O}$ contents (4.77-4.79 wt.\%). In the $\mathrm{SiO}_{2}$ versus $\mathrm{ALK}$ diagram, the granitoids plot into the subalkaline granite field (Figure 9a). In the $\mathrm{SiO}_{2} \mathrm{vs} \mathrm{K}_{2} \mathrm{O}$ diagram and $\mathrm{Si}$ vs. ALK-Ca diagram, all the samples plot into the high-K calc-alkaline field (Figure 9b,d). In the A/NK-A/CNK diagram, samples plot into the peraluminous field, implying that the granitoids of the Chuankou ore field belong to the high-K calc-alkaline and peraluminous series (Figure 9c).
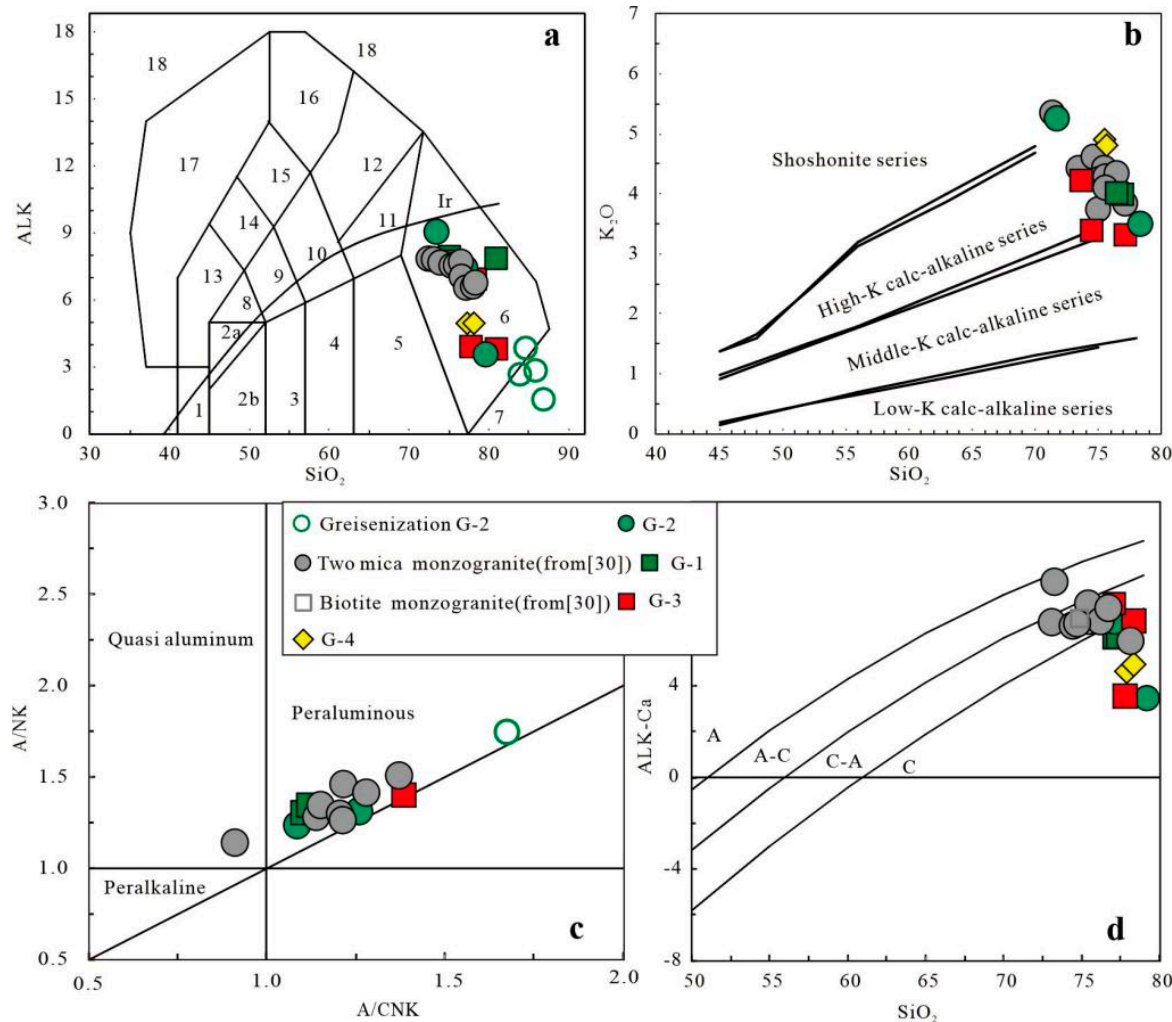

Figure 9. Major elements diagrams of granitoids from Chuankou ore field. (a) ALK $\left(\mathrm{ALK}=\mathrm{Na}_{2} \mathrm{O}\right.$ $+\mathrm{K}_{2} \mathrm{O}$ ) versus $\mathrm{SiO}_{2}$ diagrams, (b) $\mathrm{K}_{2} \mathrm{O}$ versus $\mathrm{SiO}_{2}$ diagrams, (c) A/NK versus $\mathrm{A} / \mathrm{CNK}$ diagrams, (d) ALK-Ca versus $\mathrm{SiO}_{2}$ diagrams.

G-1 shows a typical light REE-enriched pattern with an obvious negative Eu anomaly $(\delta \mathrm{Eu}=0.28$ to 0.30$)$ (Figure $10 \mathrm{~b})$. The values of $\mathrm{La}_{\mathrm{N}} / \mathrm{Yb}_{\mathrm{N}}$ range from 3.92 to 4.18 , indicating moderate fractionation between HREEs and LREEs. Zr, Hf, Th, and U are enriched and $\mathrm{Ba}, \mathrm{Sr}, \mathrm{P}$, and $\mathrm{Ti}$ are depleted in Figure $11 \mathrm{~b}$. The $\mathrm{Rb} / \mathrm{Sr}$ ratios range from 7.91 to 7.96 , the $\mathrm{K} / \mathrm{Rb}$ ratios range from 83.51 to 84.19 , the $\mathrm{Rb} / \mathrm{Ba}$ ratios range from 2.60 to 2.65, and the value of $\mathrm{Zr}+\mathrm{Nb}+\mathrm{Y}+\mathrm{Ce}$ ranges from 181.03 to $187.43 \mathrm{ppm}$. The chondrite-normalized REE patterns of G-2 exhibit a strongly negative Eu anomaly ( $\delta \mathrm{Eu}=0.06 \sim 0.23$ ) (Figure 10a). The values of $\mathrm{La}_{\mathrm{N}} / \mathrm{Yb}_{\mathrm{N}}$ range from 0.93 to 2.39. $\mathrm{Rb}, \mathrm{Hf}$, and $\mathrm{U}$ are enriched and $\mathrm{Ba}, \mathrm{Sr}$, and $\mathrm{Ti}$ are depleted (Figure 11a). $\mathrm{Rb} / \mathrm{Sr}$ ratios vary from 14.26 to $34.48, \mathrm{~K} / \mathrm{Rb}$ ratios range from 42.96 to 73.83 , and $\mathrm{Rb} / \mathrm{Ba}$ ratios range from 6.04 to 7.88 . The value of $\mathrm{Zr}+\mathrm{Nb}+\mathrm{Y}+\mathrm{Ce}$ ranges from 97.9 to $155.75 \mathrm{ppm}$. The chondrite-normalized REE patterns of G-3 are similar to G-2. The $\delta$ Eu values of G-3 range from 0.02 to 0.45 , and the values of $\mathrm{La}_{\mathrm{N}} / \mathrm{Yb}_{\mathrm{N}}$ range from 0.75 to 6.87 (Figure 10c). Rb, Hf, and Th are enriched and $\mathrm{Ba}, \mathrm{Sr}, \mathrm{P}$, and $\mathrm{Ti}$ are depleted (Figure 11c). The $\mathrm{Rb} / \mathrm{Sr}$ ratios vary from 28.6 to $78.58, \mathrm{~K} / \mathrm{Rb}$ ratios range from 35.32 to 
87.73, $\mathrm{Rb}$ / Ba ratios range from 4.50 to 17.16 , and the values of $\mathrm{Zr}+\mathrm{Nb}+\mathrm{Y}+\mathrm{Ce}$ are from 127.5 to $158.69 \mathrm{ppm}$. G-4 has an obvious negative Eu anomaly with the $\delta \mathrm{Eu}$ values ranging from 0.36 to 0.38 , and the $(\mathrm{La} / \mathrm{Yb})_{\mathrm{N}}$ values from 18.91 to 19.02 (Figure 10d). Zr, Hf, Rb, Th, and $\mathrm{U}$ are enriched and $\mathrm{Ba}, \mathrm{Sr}, \mathrm{P}$, and $\mathrm{Ti}$ are depleted (Figure $11 \mathrm{~d}$ ). The $\mathrm{Rb} / \mathrm{Sr}$ ratios vary from 13.06 to $13.20, \mathrm{~K} / \mathrm{Rb}$ ratios range from 87.19 to 87.83 , and $\mathrm{Rb} / \mathrm{Ba}$ ratios range from 1.32 to 1.34 . The values of $\mathrm{Zr}+\mathrm{Nb}+\mathrm{Y}+\mathrm{Ce}$ range from 281.59 to $284.42 \mathrm{ppm}$.
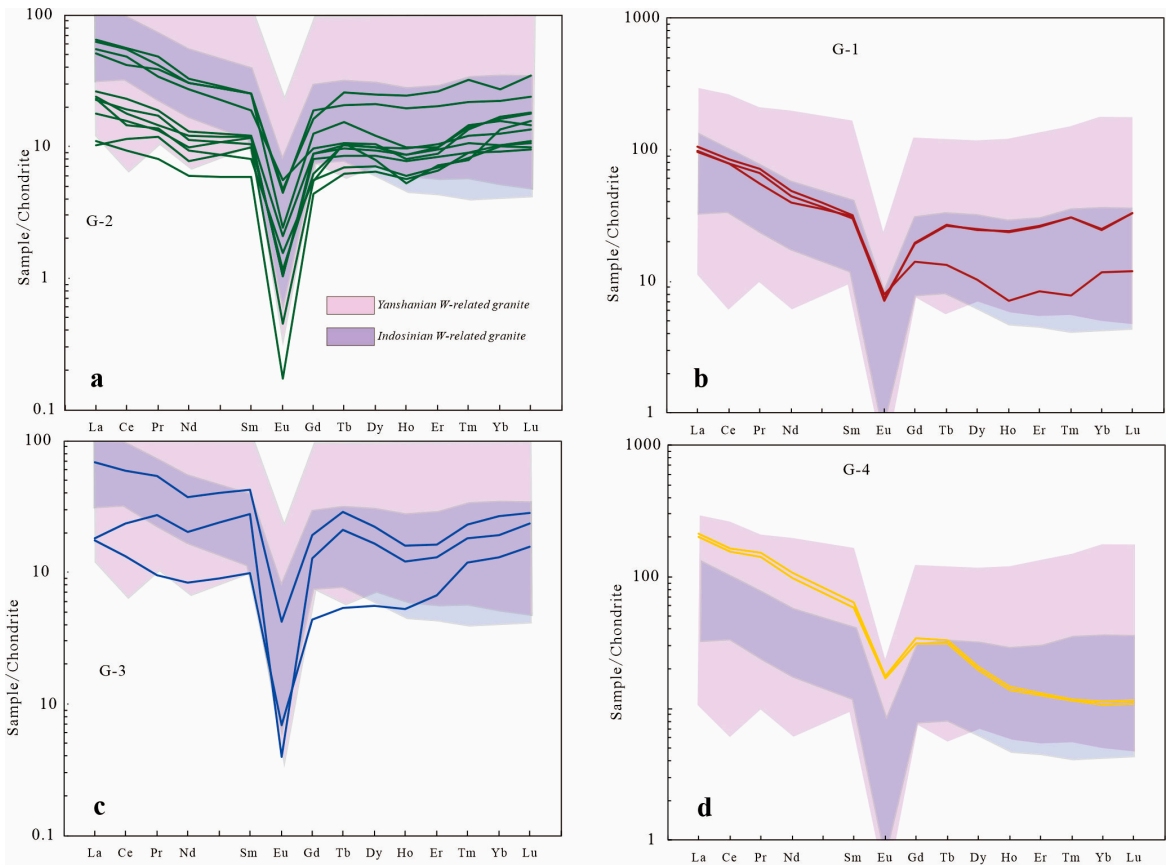

Figure 10. Chondrite-normalized REE patterns of granitoids in Chuankou ore field (Standardized values from [48]). (a) G-2; (b) G-1; (c) G-3, (d) G-4.
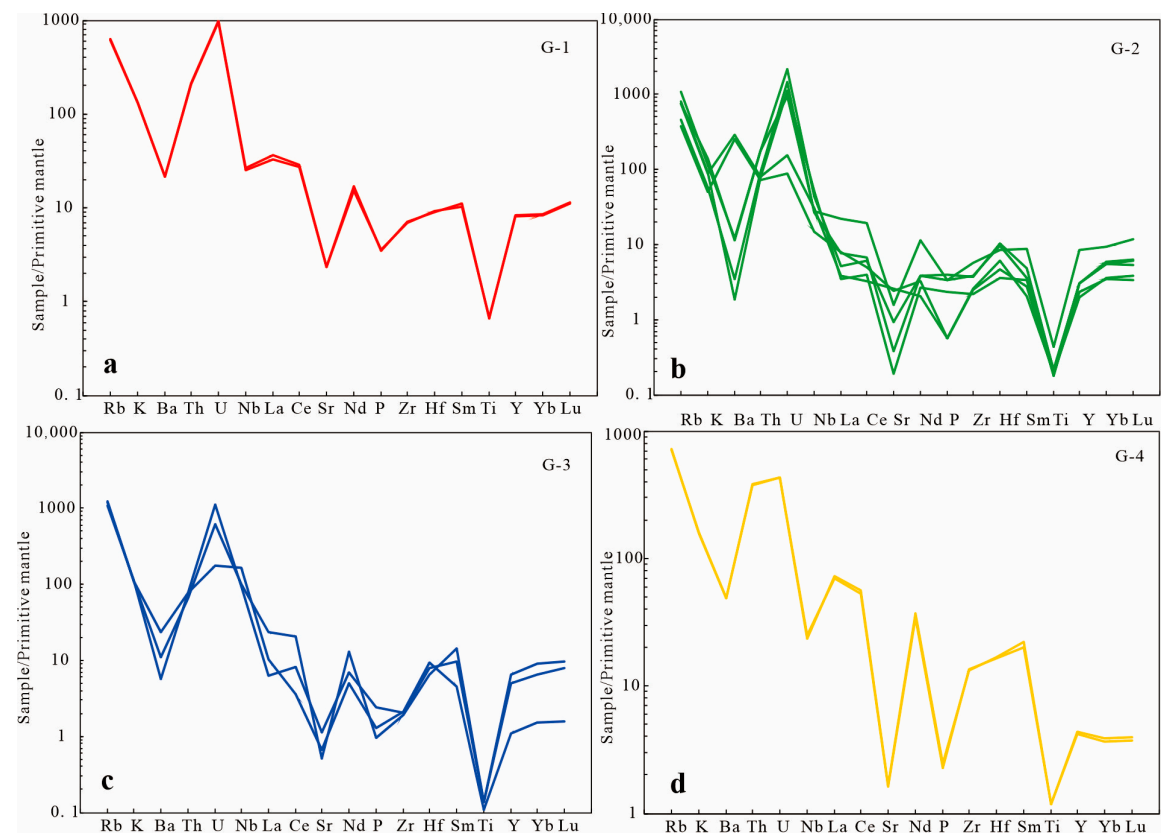

Figure 11. Primitivemantle-normalized trace element patterns of intrusions in Chuankou ore field (Standardized values from [48]). (a) G-1; (b) G-2; (c) G-3, (d) G-4. 


\subsection{Zircon Geochemistry and $\mathrm{Ce}^{4+} / \mathrm{Ce}^{3+}$ Ratios}

The trace element compositions of zircon grains from the granitoids in the Chuankou ore field are shown in Table 4. Most of the $\mathrm{Ti}$, $\mathrm{Sr}$, and $\mathrm{Ta}$ contents of zircon grains are much closer to the values range proposed by Hoskin and Schaltegger [49] (Nb: up to $62 \mathrm{ppm}$; $\mathrm{Sr} \leq 3 \mathrm{ppm}$; Ti: up to $75 \mathrm{ppm}$ ), which could be interpreted as normal magmatic zircon with various microscopic mineral inclusions, such as rutile and ferrotapiolite [50]. The $\Sigma$ REE contents of G-1 range from 787.62 to $2080.54 \mathrm{ppm}$, those of G-2 range from 935.37 to $11,137.50 \mathrm{ppm}$, and those of G-3 range from 1387.73 to $4694.70 \mathrm{ppm}$. The chondritenormalized REE patterns reveal an obvious enrichment of HREEs and depletion of LREEs but depletion of LREEs and connect with a magmatic origin [51]. All samples commonly show positive $\mathrm{Ce}$ anomalies and negative Eu anomalies in the zircons (Figure 12). However, there is an obvious difference in the degree of $\mathrm{Ce}$ and $\mathrm{Eu}$ anomalies in that G-1 and $\mathrm{G}-2$ contain more negative $\mathrm{Eu}$ anomalies and positive $\mathrm{Ce}$ anomalies $(\delta \mathrm{Eu}=0.03-0.28$; $\delta \mathrm{Ce}=1.56-189.58)$ than $\mathrm{G}-3(\delta \mathrm{Eu}=0.12-0.47$, with a value of $1.41 ; \delta \mathrm{Ce}=1.04-8.81)$. Despite this difference, all zircon grains in the study appear to be magmatic in origin and do not show geochemical evidence of metamorphic, hydrothermal overprinting or radiationinduced damage.
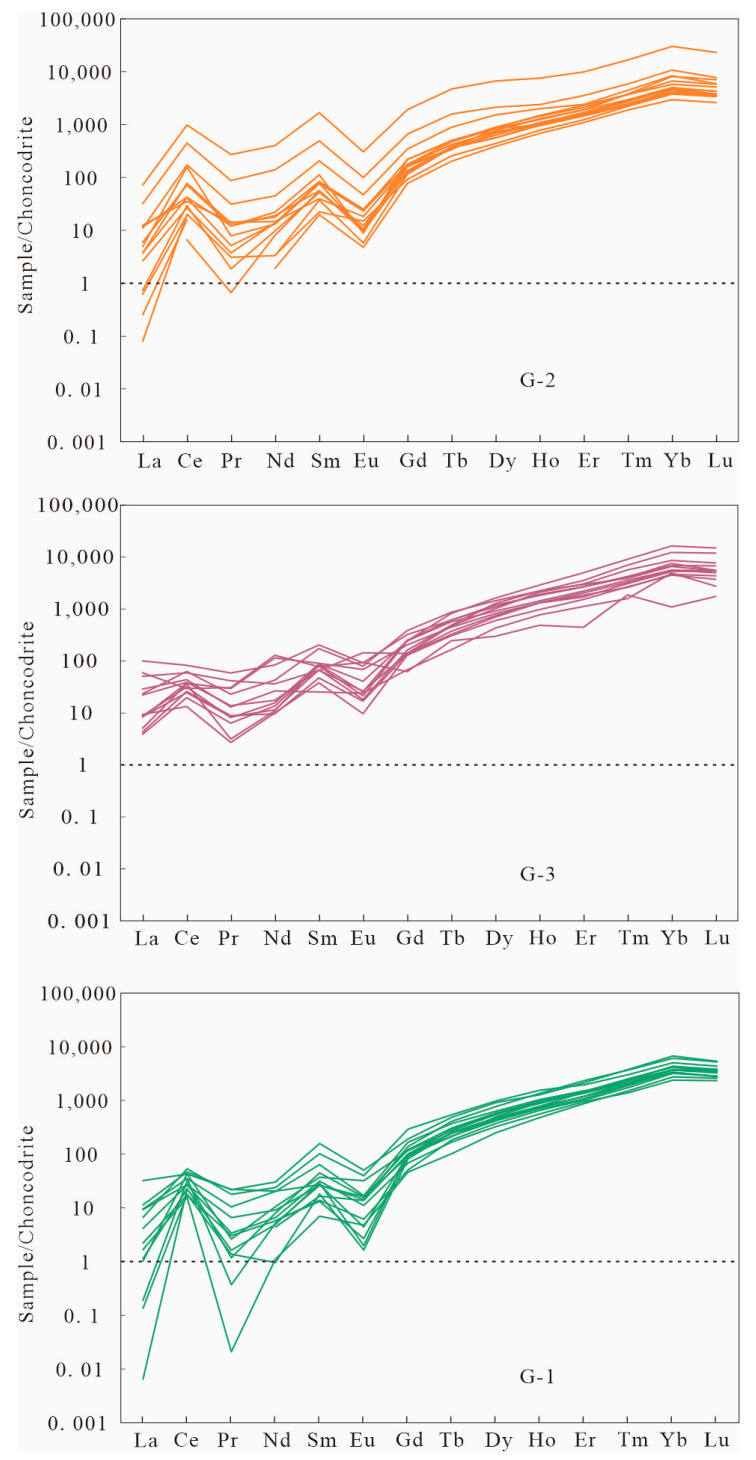

Figure 12. Chondrite-normalized REE patterns of zircon grains from igneous rocks in Chuankou ore field (Standardized value from [48]). 
Table 4. Trace element composition (ppm) of zircons of granitoids from Chuankou ore field.

\begin{tabular}{|c|c|c|c|c|c|c|c|c|c|c|c|c|c|c|c|}
\hline \multirow[t]{2}{*}{ Sample } & $\begin{array}{c}\text { HNCK- } \\
2-01\end{array}$ & $\begin{array}{c}\text { HNCK- } \\
2-02\end{array}$ & $\begin{array}{c}\text { HNCK- } \\
2-03\end{array}$ & $\begin{array}{c}\text { HNCK- } \\
2-04\end{array}$ & $\begin{array}{c}\text { HNCK- } \\
2-05\end{array}$ & $\begin{array}{c}\text { HNCK- } \\
2-06\end{array}$ & $\begin{array}{c}\text { HNCK- } \\
2-07\end{array}$ & $\begin{array}{c}\text { HNCK- } \\
2-08\end{array}$ & $\begin{array}{c}\text { HNCK- } \\
2-09\end{array}$ & $\begin{array}{c}\text { HNCK- } \\
2-10\end{array}$ & $\begin{array}{c}\text { HNCK- } \\
2-11\end{array}$ & $\begin{array}{c}\text { HNCK- } \\
2-12\end{array}$ & $\begin{array}{c}\text { HNCK- } \\
2-13\end{array}$ & $\begin{array}{c}\text { HNCK- } \\
2-14\end{array}$ & $\begin{array}{c}\text { HNCK- } \\
2-15\end{array}$ \\
\hline & \multicolumn{15}{|c|}{ G-2 } \\
\hline $\mathrm{La}$ & 0.90 & 0.02 & 1.17 & 0.06 & 7.56 & 0.17 & 0.88 & 2.73 & 2.62 & 17.13 & 0.00 & 0.63 & 2.96 & 0.15 & 1.41 \\
\hline $\mathrm{Ce}$ & 25.29 & 12.40 & 96.62 & 9.68 & 273.32 & 17.91 & 47.45 & 25.89 & 106.06 & 601.34 & 4.04 & 16.28 & 21.47 & 11.88 & 44.03 \\
\hline $\operatorname{Pr}$ & 0.47 & 0.29 & 0.73 & 0.00 & 8.10 & 0.17 & 1.27 & 1.12 & 2.89 & 25.23 & 0.06 & 0.35 & 1.33 & 0.00 & 1.13 \\
\hline $\mathrm{Nd}$ & 6.10 & 1.52 & 6.01 & 1.56 & 63.79 & 4.47 & 8.15 & 8.71 & 20.54 & 183.58 & 3.71 & 6.33 & 6.68 & 0.87 & 10.12 \\
\hline $\mathrm{Sm}$ & 11.37 & 5.50 & 11.35 & 3.30 & 72.62 & 7.66 & 12.39 & 8.34 & 30.36 & 248.71 & 8.31 & 11.60 & 5.85 & 2.93 & 16.63 \\
\hline $\mathrm{Eu}$ & 1.33 & 0.32 & 0.56 & 0.84 & 5.62 & 0.69 & 1.42 & 0.60 & 2.65 & 17.20 & 0.54 & 1.30 & 1.03 & 0.27 & 0.50 \\
\hline $\mathrm{Tb}$ & 18.07 & 13.11 & 15.08 & 9.24 & 57.49 & 13.31 & 12.61 & 13.04 & 32.12 & 170.15 & 13.92 & 17.00 & 12.09 & 7.30 & 17.34 \\
\hline Dy & 213.53 & 138.20 & 178.97 & 107.65 & 521.08 & 137.97 & 170.44 & 154.84 & 375.47 & 1638.95 & 190.06 & 207.54 & 213.79 & 95.04 & 220.01 \\
\hline Ho & 84.31 & 54.48 & 59.35 & 44.01 & 135.41 & 57.93 & 55.60 & 61.58 & 112.44 & 428.51 & 72.73 & 69.98 & 54.12 & 38.57 & 81.74 \\
\hline Er & 371.80 & 236.53 & 273.48 & 196.59 & 567.00 & 251.59 & 244.81 & 262.17 & 384.47 & 1588.06 & 304.01 & 304.97 & 232.52 & 174.72 & 336.47 \\
\hline $\mathrm{Tm}$ & 91.57 & 58.63 & 70.25 & 54.92 & 145.73 & 58.21 & 60.87 & 66.32 & 110.18 & 413.87 & 93.74 & 73.19 & 57.93 & 46.50 & 91.92 \\
\hline $\mathrm{Yb}$ & 933.18 & 737.00 & 764.00 & 606.80 & 1719.15 & 650.12 & 671.90 & 776.50 & 1353.13 & 4850.35 & 1309.38 & 809.60 & 617.41 & 477.71 & 1077.43 \\
\hline $\mathrm{Lu}$ & 126.35 & 87.38 & 93.20 & 83.53 & 190.45 & 88.06 & 93.70 & 104.75 & 145.25 & 572.73 & 173.69 & 104.92 & 85.85 & 64.08 & 142.00 \\
\hline $\mathrm{Ta}$ & 2.56 & 1.83 & 4.58 & 3.59 & 8.22 & 1.78 & 3.87 & 4.39 & 8.92 & 14.89 & 14.54 & 1.53 & 1.93 & 2.19 & 6.70 \\
\hline $\mathrm{Nb}$ & 3.86 & 3.40 & 5.70 & 4.75 & 36.24 & 3.24 & 4.71 & 5.96 & 15.91 & 111.73 & 10.06 & 2.75 & 2.21 & 2.58 & 7.51 \\
\hline Sn & 1.22 & 0.70 & 1.60 & 1.27 & 0.00 & 0.25 & 1.35 & 0.49 & 0.97 & 2.36 & 10.68 & 0.60 & 1.30 & 0.50 & 0.00 \\
\hline $\mathrm{Ce}^{4+} / \mathrm{Ce}^{3+}$ & 8.56 & 18.23 & 32.64 & 18.92 & 4.69 & 8.62 & 11.12 & 7.50 & 7.80 & 2.97 & 2.72 & 4.51 & 7.75 & 36.67 & 8.92 \\
\hline$\delta \mathrm{Eu}$ & 0.16 & 0.08 & 0.08 & 0.26 & 0.17 & 0.13 & 0.20 & 0.10 & 0.17 & 0.17 & 0.11 & 0.18 & 0.18 & 0.10 & 0.05 \\
\hline$\left(\mathrm{Ce} / \mathrm{Ce}^{*}\right) \mathrm{D}$ & 22.52 & 45.35 & 78.89 & 46.97 & 13.34 & 22.69 & 28.54 & 20.03 & 20.70 & 9.27 & 8.77 & 13.00 & 20.62 & 88.82 & 23.36 \\
\hline \multirow[t]{2}{*}{ Sample } & $\begin{array}{c}\text { HNCK10- } \\
14-01\end{array}$ & $\begin{array}{l}\text { HNCK10- } \\
14-02\end{array}$ & $\begin{array}{l}\text { HNCK10- } \\
14-03\end{array}$ & $\begin{array}{c}\text { HNCK10- } \\
14-04\end{array}$ & $\begin{array}{c}\text { HNCK10- } \\
14-05\end{array}$ & $\begin{array}{c}\text { HNCK10- } \\
14-06\end{array}$ & $\begin{array}{c}\text { HNCK10- } \\
14-07\end{array}$ & $\begin{array}{l}\text { HNCK10- } \\
14-08\end{array}$ & $\begin{array}{c}\text { HNCK10- } \\
14-09\end{array}$ & $\begin{array}{c}\text { HNCK10- } \\
14-10\end{array}$ & $\begin{array}{c}\text { HNCK10- } \\
14-11\end{array}$ & $\begin{array}{c}\text { HNCK10- } \\
14-12\end{array}$ & $\begin{array}{c}\text { HNCK10- } \\
14-13\end{array}$ & $\begin{array}{c}\text { HNCK10- } \\
14-14\end{array}$ & $\begin{array}{c}\text { HNCK10- } \\
14-15\end{array}$ \\
\hline & \multicolumn{15}{|c|}{ G-1 } \\
\hline $\mathrm{La}$ & 0.52 & 0.03 & 0.24 & 1.57 & 0.40 & 7.64 & 0.04 & 2.68 & 2.25 & 0.00 & 0.00 & 0.27 & 2.21 & 0.00 & 0.98 \\
\hline $\mathrm{Ce}$ & 10.67 & 10.97 & 26.27 & 32.82 & 9.57 & 26.02 & 19.43 & 28.54 & 21.44 & 21.54 & 13.73 & 13.84 & 16.58 & 19.18 & 16.27 \\
\hline $\operatorname{Pr}$ & 0.28 & 0.13 & 0.24 & 1.66 & 0.00 & 2.06 & 0.11 & 2.03 & 0.97 & 0.03 & 0.15 & 0.00 & 0.61 & 0.00 & 0.31 \\
\hline $\mathrm{Nd}$ & 2.62 & 0.44 & 4.80 & 10.94 & 0.51 & 9.37 & 3.64 & 13.88 & 9.27 & 2.37 & 2.25 & 2.65 & 4.17 & 2.00 & 3.00 \\
\hline $\mathrm{Sm}$ & 2.05 & 2.69 & 5.51 & 15.05 & 1.03 & 4.01 & 6.62 & 23.31 & 9.44 & 3.90 & 4.25 & 1.95 & 3.86 & 2.45 & 4.65 \\
\hline $\mathrm{Eu}$ & 0.35 & 0.09 & 1.80 & 2.23 & 0.27 & 0.25 & 0.89 & 2.83 & 0.95 & 0.81 & 0.11 & 0.15 & 0.90 & 0.77 & 0.62 \\
\hline $\mathrm{Gd}$ & 10.09 & 16.25 & 23.16 & 57.96 & 9.22 & 19.40 & 24.23 & 38.13 & 33.10 & 27.64 & 19.85 & 18.41 & 17.91 & 20.05 & 13.61 \\
\hline
\end{tabular}


Table 4. Cont.

\begin{tabular}{|c|c|c|c|c|c|c|c|c|c|c|c|c|c|c|c|}
\hline $\mathrm{Tb}$ & 6.72 & 9.30 & 9.99 & 19.55 & 3.71 & 7.68 & 11.09 & 17.58 & 13.44 & 14.88 & 10.38 & 7.89 & 8.84 & 8.93 & 6.11 \\
\hline Dy & 92.16 & 117.29 & 135.00 & 242.08 & 62.09 & 122.91 & 144.72 & 225.49 & 155.97 & 190.67 & 141.33 & 103.92 & 108.16 & 109.14 & 80.57 \\
\hline Ho & 37.02 & 44.25 & 53.61 & 87.34 & 27.26 & 51.60 & 55.36 & 72.33 & 58.82 & 76.74 & 49.81 & 40.12 & 44.33 & 41.55 & 31.92 \\
\hline Er & 163.62 & 189.23 & 218.28 & 313.63 & 140.60 & 225.78 & 227.03 & 344.23 & 242.08 & 375.28 & 237.95 & 172.02 & 230.55 & 193.13 & 152.35 \\
\hline $\mathrm{Tm}$ & 43.16 & 48.75 & 53.37 & 74.80 & 37.36 & 58.04 & 57.97 & 93.31 & 52.15 & 91.49 & 62.76 & 47.36 & 61.25 & 52.23 & 34.55 \\
\hline $\mathrm{Yb}$ & 513.31 & 589.04 & 611.02 & 811.23 & 440.76 & 601.55 & 697.92 & 1084.79 & 595.18 & 980.66 & 667.68 & 539.34 & 686.35 & 534.13 & 385.14 \\
\hline $\mathrm{Lu}$ & 69.75 & 82.74 & 88.76 & 107.07 & 63.68 & 84.37 & 88.60 & 131.40 & 80.08 & 128.82 & 87.25 & 68.95 & 93.77 & 69.17 & 57.53 \\
\hline $\mathrm{Ta}$ & 2.35 & 2.89 & 2.60 & 2.02 & 2.24 & 3.26 & 2.65 & 6.76 & 1.23 & 5.00 & 3.12 & 2.62 & 3.45 & 2.66 & 2.27 \\
\hline $\mathrm{Nb}$ & 2.74 & 2.88 & 4.33 & 4.33 & 2.80 & 4.62 & 4.12 & 9.16 & 1.13 & 6.58 & 3.21 & 3.05 & 4.16 & 3.08 & 3.58 \\
\hline W & 208.89 & 3.06 & 0.29 & 1113.80 & 1.34 & 2929.50 & 0.08 & 826.84 & 0.00 & 43.12 & 6.10 & 0.45 & 53.45 & 0.31 & 259.16 \\
\hline Sn & 4.54 & 2.00 & 1.44 & 1.04 & 2.44 & 0.00 & 1.31 & 0.83 & 1.23 & 0.00 & 0.82 & 4.83 & 4.57 & 2.19 & 2.44 \\
\hline$\delta \mathrm{Eu}$ & 0.19 & 0.03 & 0.42 & 0.20 & 0.18 & 0.07 & 0.19 & 0.29 & 0.15 & 0.17 & 0.03 & 0.05 & 0.28 & 0.23 & 0.22 \\
\hline$\left(\mathrm{Ce} / \mathrm{Ce}^{*}\right) \mathrm{D}$ & 47.44 & 155.33 & 38.87 & 12.96 & 222.34 & 29.65 & 32.91 & 10.21 & 12.19 & 80.72 & 45.01 & 52.91 & 38.77 & 79.76 & 34.94 \\
\hline Sample & $\begin{array}{l}\text { HNCK10- } \\
\text { 10a-1 }\end{array}$ & $\begin{array}{c}\text { HNCK10- } \\
\text { 10a-2 }\end{array}$ & $\begin{array}{c}\text { HNCK10- } \\
\text { 10a-3 }\end{array}$ & $\begin{array}{c}\text { HNCK10- } \\
\text { 10a-4 }\end{array}$ & $\begin{array}{c}\text { HNCK10- } \\
\text { 10a-5 }\end{array}$ & $\begin{array}{l}\text { HNCK10- } \\
\text { 10a-6 }\end{array}$ & $\begin{array}{c}\text { HNCK10- } \\
10 a-10\end{array}$ & $\begin{array}{c}\text { HNCK10- } \\
\text { 10a-11 }\end{array}$ & $\begin{array}{c}\text { HNCK10- } \\
\text { 10a-12 }\end{array}$ & $\begin{array}{l}\text { HNCK10- } \\
\text { 10a-13 }\end{array}$ & $\begin{array}{c}\text { HNCK10- } \\
\text { 10a-14 }\end{array}$ & $\begin{array}{l}\text { HNCK10- } \\
\text { 10a-15 }\end{array}$ & & & \\
\hline \multicolumn{16}{|c|}{ G-3 } \\
\hline $\mathrm{La}$ & 1.58 & 23.77 & 1.03 & 5.54 & 2.02 & 1.20 & 2.21 & 0.92 & 5.27 & 12.01 & 1.99 & 2.09 & & & \\
\hline $\mathrm{Ce}$ & 22.73 & 50.62 & 15.88 & 38.46 & 15.11 & 22.28 & 8.16 & 12.11 & 23.80 & 36.39 & 22.83 & 19.39 & & & \\
\hline $\operatorname{Pr}$ & 0.59 & 5.47 & 0.83 & 2.13 & 0.78 & 0.29 & 0.25 & 0.59 & 1.29 & 3.88 & 2.76 & 0.75 & & & \\
\hline $\mathrm{Nd}$ & 11.71 & 38.38 & 4.42 & 19.55 & 5.27 & 5.05 & 4.58 & 6.23 & 8.03 & 16.72 & 52.38 & 7.18 & & & \\
\hline $\mathrm{Gd}$ & 49.57 & 64.22 & 27.11 & 76.35 & 31.97 & 49.87 & 25.61 & 48.36 & 27.80 & 27.79 & 40.47 & 48.53 & & & \\
\hline $\mathrm{Tb}$ & 22.61 & 22.02 & 11.71 & 31.17 & 17.52 & 22.23 & 11.07 & 29.72 & 13.43 & 17.08 & 16.11 & 20.53 & & & \\
\hline Dy & 279.87 & 291.96 & 172.42 & 354.28 & 315.86 & 269.62 & 147.22 & 399.42 & 183.43 & 249.03 & 200.44 & 223.72 & & & \\
\hline Ho & 100.58 & 102.80 & 74.13 & 125.47 & 125.25 & 107.39 & 54.88 & 162.62 & 73.58 & 118.46 & 74.50 & 80.77 & & & \\
\hline Er & 429.13 & 424.07 & 327.38 & 476.88 & 565.61 & 432.73 & 244.54 & 798.37 & 273.39 & 486.20 & 295.84 & 352.58 & & & \\
\hline $\mathrm{Tm}$ & 108.85 & 100.56 & 80.92 & 96.75 & 173.30 & 104.45 & 66.14 & 223.92 & 76.40 & 139.04 & 64.09 & 88.51 & & & \\
\hline $\mathrm{Yb}$ & 1114.37 & 1209.43 & 903.11 & 1061.55 & 1977.23 & 1101.13 & 724.86 & 2630.70 & 843.59 & 1380.64 & 749.70 & 901.04 & & & \\
\hline $\mathrm{Lu}$ & 148.83 & 136.98 & 133.65 & 133.95 & 292.72 & 167.34 & 92.04 & 368.63 & 133.89 & 189.78 & 107.65 & 122.48 & & & \\
\hline $\mathrm{Ta}$ & 1.49 & 5.47 & 3.02 & 1.41 & 7.61 & 2.76 & 2.25 & 15.80 & 2.99 & 7.23 & 0.78 & 3.11 & & & \\
\hline $\mathrm{Nb}$ & 3.85 & 14.32 & 4.10 & 5.84 & 7.22 & 3.36 & 3.02 & 8.24 & 4.29 & 22.01 & 2.04 & 3.16 & & & \\
\hline W & 21.48 & 5517.37 & 793.71 & 335.84 & 77.92 & 503.39 & 332.05 & 47.46 & 637.48 & 2347.57 & 53.20 & 165.03 & & & \\
\hline Sn & 11.94 & 10.89 & 0.17 & 39.41 & 36.37 & 13.69 & 1.00 & 2.46 & 9.40 & 20.39 & 1.43 & 41.27 & & & \\
\hline $\mathrm{Ce}^{4+} / \mathrm{Ce}^{3+}$ & 3.73 & 1.73 & 10.83 & 2.27 & 11.52 & -0.23 & 4.49 & 7.03 & 7.74 & 9.39 & 0.33 & 4.58 & & & \\
\hline$\delta \mathrm{Eu}$ & 0.27 & 0.35 & 0.18 & 0.29 & 0.16 & 1.34 & 0.12 & 0.13 & 0.26 & 1.41 & 0.47 & 0.25 & & & \\
\hline$\left(\mathrm{Ce} / \mathrm{Ce}^{*}\right) \mathrm{D}$ & 11.15 & 6.44 & 27.89 & 7.70 & 29.51 & 1.83 & 12.96 & 18.95 & 20.60 & 24.48 & 3.14 & 13.16 & & & \\
\hline
\end{tabular}


Ballard et al. [52] proposed a detailed calculation formula for the $\mathrm{Ce}^{4+} / \mathrm{Ce}^{3+}$ ratio:

$$
\mathrm{Ce}^{4+} / \mathrm{Ce}^{3+}=\left(\mathrm{Ce}_{\text {melt }}-\mathrm{Ce}_{\text {zircon }} / \mathrm{D}_{\mathrm{Ce}^{3+}}^{\text {zircon } / \text { melt }}\right) /\left(\mathrm{Ce}_{\text {zircon }} / \mathrm{D}_{\mathrm{Ce}^{4+}}^{\text {zircon } / \text { melt }}-\mathrm{Ce}_{\text {melt }}\right)
$$

The zircon-melt partition coefficients for $\mathrm{Ce}^{3+}$ and $\mathrm{Ce}^{4+}$ were estimated using the model described by Ballard et al. and Zhang et al. [52,53]. The Ce $e_{\text {melt }}$ value is approximately equal to the $\mathrm{Zr}$ content of bulk rocks, and the parameters of $\mathrm{D}_{\mathrm{Ce}}^{\text {zircon/melt }}$ and $\mathrm{D}_{\mathrm{Ce}^{4+}}^{\text {zircon/melt }}$ can be deduced from the lattice strain model proposed by Blundy and Wood [54]. Calculated $\mathrm{Ce}^{4+} / \mathrm{Ce}^{3+}$ ratios of $\mathrm{G}-1$ range from 3.33 to $93.28, \mathrm{Ce}^{4+} / \mathrm{Ce}^{3+}$ ratios of $\mathrm{G}-2$ range from 2.72 to 36.67 , and $\mathrm{Ce}^{4+} / \mathrm{Ce}^{3+}$ ratios of $\mathrm{G}-3$ range from 0.33 to 11.52 .

\section{4. $\mathrm{Rb}$-Sr and Sm-Nd Isotope}

Granitoids from the Chuankou ore field contain low Sr contents (varying from 14.16 to $55 \mathrm{ppm}$ ) and high ${ }^{87} \mathrm{Rb} /{ }^{86} \mathrm{Sr}$ ratios (13.56-152.35) (Table 5). The initial ${ }^{87} \mathrm{Sr} /{ }^{86} \mathrm{Sr}, \mathrm{T}_{\mathrm{DM} 2}$, $\varepsilon_{\mathrm{Nd}}(\mathrm{t})$ are calculated using zircon $\mathrm{U}-\mathrm{Pb}$ ages of $202.9 \mathrm{Ma}$ for G-3, $224 \mathrm{Ma}$ for G-2, and $230 \mathrm{Ma}$ for G-1. The calculated initial ${ }^{87} \mathrm{Sr} /{ }^{86} \mathrm{Sr}$ for G-1 is 0.72109 , that for G-2 ranges from 0.67995 to 0.70851 , and that for G-3 varies from 0.74915 to 0.85226 . The ${ }^{143} \mathrm{Nd} /{ }^{144} \mathrm{Nd}$ values of G-3 range from 0.512122 to 0.512303 , and the calculated $\varepsilon_{\mathrm{Nd}}(\mathrm{t})$ values range from -10.07 to -6.53 . The calculated $\mathrm{T}_{\mathrm{DM} 2}$ varies from 1471 to $1669 \mathrm{Ma}$ and the ${ }^{143} \mathrm{Nd} /{ }^{144} \mathrm{Nd}$ value of G-1 is 0.512086; the calculated $\varepsilon_{\mathrm{Nd}}(\mathrm{t})$ value is -10.77 and the $\mathrm{T}_{\mathrm{DM} 2}$ value is $2090 \mathrm{Ma}$. Moreover, the ${ }^{143} \mathrm{Nd} /{ }^{144} \mathrm{Nd}$ values of G-2 vary from 0.512161 to 0.512255 , calculated $\varepsilon_{\mathrm{Nd}}(\mathrm{t})$ values range from -9.09 to -7.47 , and $\mathrm{T}_{\mathrm{DM} 2}$ values range from 1684 to $1764 \mathrm{Ma}$.

Table 5. The Sr-Nd isotopic composition of samples of granitoids from Chuankou ore field.

\begin{tabular}{|c|c|c|c|c|c|c|c|c|}
\hline Sample & Lithology & $\begin{array}{l}\text { Age } \\
\text { (Ma) }\end{array}$ & $\begin{array}{c}\mathbf{R b} \\
(\mathrm{ppm})\end{array}$ & $\begin{array}{c}\mathrm{Sr} \\
(\mathrm{ppm})\end{array}$ & $\frac{{ }^{87} \mathrm{Rb}}{{ }^{86} \mathrm{Sr}}$ & $\frac{{ }^{87} \mathrm{Sr}}{{ }^{86} \mathrm{Sr}}$ & $\mathbf{I}_{\mathrm{Sr}}$ & $\varepsilon_{S r}(t)$ \\
\hline HNCK10-9 & G-3 & 203 & 744.72 & 14.155 & 152.3464 & 1.292048 & 0.85226 & 2101.5 \\
\hline HNCK10-5 & G-3 & 203 & 682.86 & 23.87 & 82.8378 & 0.98828 & 0.74915 & 637.4 \\
\hline HNCK10-14 & G-1 & 230 & 395.14 & 49.91 & 22.9252 & 0.796091 & 0.72109 & 239.4 \\
\hline HNCK1 & & 224 & 469 & 55 & 24.6922 & 0.784304 & 0.70564 & 19.9 \\
\hline HNCK2 & G-2 & 224 & 236 & 50.4 & 13.5591 & 0.751712 & 0.70851 & 60.7 \\
\hline HNCK3 & & 224 & 676 & 19.6 & 99.8711 & 0.998122 & 0.67995 & -344.9 \\
\hline $\begin{array}{c}\mathrm{Sm} \\
(\mathrm{ppm})\end{array}$ & $\begin{array}{c}\mathrm{Nd} \\
(\mathrm{ppm})\end{array}$ & $\frac{147 \mathrm{Sm}}{{ }^{144} \mathrm{Nd}}$ & $\frac{143 \mathrm{Nd}}{{ }^{144} \mathrm{Nd}}$ & $\mathbf{I}_{\mathrm{Nd}}$ & $\mathrm{T}_{\mathrm{DM} 2}$ & $\varepsilon_{\mathrm{Nd}}(0)$ & $\mathcal{E}_{\mathrm{Nd}}(\mathrm{t})$ & $f_{\mathrm{Sm} / \mathrm{Nd}}$ \\
\hline 4.617 & 20.82 & 0.1341 & 0.512122 & 0.511944 & 1669 & -10.07 & -8.44 & -0.32 \\
\hline 1.978 & 6.649 & 0.1799 & 0.512303 & 0.512064 & 1471 & -6.53 & -6.1 & -0.09 \\
\hline 4.248 & 9.442 & 0.272 & 0.512086 & 0.511677 & 2090 & -10.77 & -12.98 & 0.38 \\
\hline 0.9 & 2.77 & 0.1964 & 0.512173 & 0.511885 & 1705 & -9.07 & -9.07 & 0 \\
\hline 1.22 & 4.39 & 0.168 & 0.512161 & 0.511915 & 1684 & -9.3 & -8.48 & -0.15 \\
\hline 2.14 & 5.13 & 0.2522 & 0.512255 & 0.511885 & 1764 & -7.47 & -9.07 & 0.28 \\
\hline
\end{tabular}

\section{Discussion}

\subsection{Magmatic Stage of the Granitoids from Chuankou Ore Field}

Bai et al. [55] proposed the formation age of Chuankou granitoids ranged from 160 to $170 \mathrm{Ma}$ and emphasized that the mineralization of $\mathrm{W}$ occurred in the early Middle Jurassic. Conflicting data by Peng et al. and Qin et al. indicate that the ore-forming age varies from 224 to $230 \mathrm{Ma}$ based on Re-Os isotopic chronology data of molybdenite [31,56]. Due to the absence of detailed field observations and efficient constraints on geochronology, the magmatic process and evolution of granitoids from the Chaunkou ore field remain unclear.

In this study, zircon $\mathrm{U}-\mathrm{Pb}$ geochoronological analysis of the four main phases (G-1-G-4) was carried out. G-1 is exposed at the depth of the Maowan and Tangjiangyuan deposits. The formation age of G-1 is $230.8 \pm 1.6 \mathrm{Ma}(\mathrm{MSWD}=0.31)$. G-2 is the dominant part and represents approximately $70 \%$ of the granitoids in size. The formation age of G-2 
is $222.1 \pm 0.56 \mathrm{Ma}$, which is similar to the results of 223.1-224.6 Ma within the allowed error range [57]. G-3 intruded into G-2 as a dyke, and two groups of concordance ages can be identified. The first group of $224.8 \pm 1.6 \mathrm{Ma}(\mathrm{MSWD}=0.047$ ) is consistent with G-2 and suggests that the zircons might be xenocrysts. The second group is $203.1 \pm 1.6 \mathrm{Ma}$ (MSWD = 7.2), representing the formation age. G-4 intruded into G-2 as larger veins with width from 0.5 to $3 \mathrm{~m}$. The field observations and analysis results confirm the conclusion that G-4 formed at $135.5 \pm 2.4 \mathrm{Ma}(\mathrm{MSWD}=1.3)$.

In summary, the Chuankou ore field experienced at least four stages of magmatism. The emplacement sequence is G-1 (phase I), G-2 (phase II), G-3 (phase III), and G-4 (phase IV).

\subsection{Genesis and Relationships Between Host Rocks and Tungsten Mineralization 6.2.1. Genesis Type}

The granitoids of the Chuankou ore field are peraluminous, reflected in both the major element ratios (A/CNK ranging from 1.110 to 4.238) and the secondary and accessory minerals (spessartine, muscovite, biotite and tourmaline). The granitoids are commonly enriched in $\mathrm{Rb}, \mathrm{Zr}, \mathrm{Hf}$, Th, and $\mathrm{U}$, whereas they are depleted in $\mathrm{Ba}, \mathrm{Sr}, \mathrm{P}$, and Ti. In addition, total alkali content ranges from 3.57 to $7.53 \mathrm{ppm}, \mathrm{FeO}^{\mathrm{T}} / \mathrm{MgO}$ ratios range from 2.40 to 13.98, and $\mathrm{Zr}+\mathrm{Nb}+\mathrm{Ce}+\mathrm{Y}$ values range from 97.9 to $284.42 \mathrm{ppm}$. These indexes are significantly lower than the global average of A-type granite (350 ppm) [58]. In the $\mathrm{Zr}+\mathrm{Nb}+\mathrm{Ce}+\mathrm{Y}$ vs. $\mathrm{ALK}$ and $\mathrm{Zr}+\mathrm{Nb}+\mathrm{Ce}+\mathrm{Y}$ vs. $\mathrm{FeO}^{\mathrm{T}} / \mathrm{MgO}$ diagrams, samples plot into the FG field suggesting that the granitoids from the Chuankou ore field have an affinity for fractionated I/S-type granite (Figure 13a,b). Thirdly, in the A (Al-Na-K)-C (Ca)-F (Fe $\left.{ }^{2+}+\mathrm{Mg}\right)$ ternary diagram, samples plot in the S-type granite field, also indicating an S-type granite affinity (Figure 14).
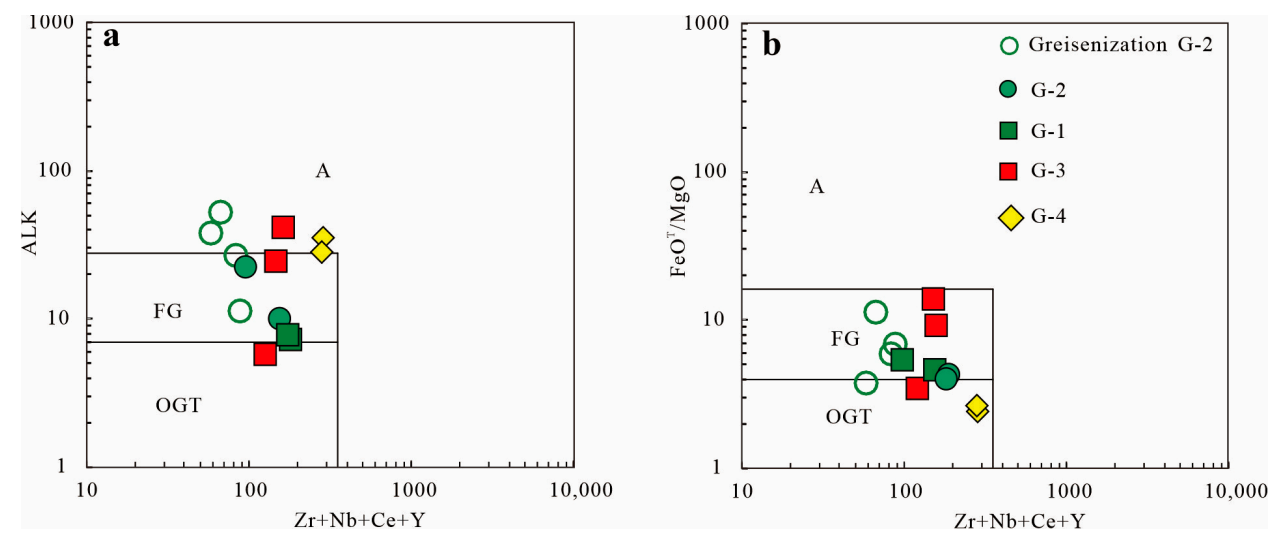

Figure 13. Genetic type discrimination diagram of intrusions in Chuankou ore field, (a) ALK versus $\mathrm{Zr}+\mathrm{Nb}+\mathrm{Ce}+\mathrm{Y}$ diagram, $(\mathbf{b}) \mathrm{FeO}^{\mathrm{T}} / \mathrm{MgO}$ versus $\mathrm{Zr}+\mathrm{Nb}+\mathrm{Ce}+\mathrm{Y}$ diagram (after [58])

\subsubsection{Origin}

In this study, granitoids from the Chuankou ore field are characterized by high ${ }^{87} \mathrm{Rb} /{ }^{86} \mathrm{Sr}$ ratios (varying from 13.5591 to 152.3436 ) and extremely high ${ }^{87} \mathrm{Sr} /{ }^{86} \mathrm{Sr}$ ratios (from 0.751712 to 1.292048 ). The initial ${ }^{87} \mathrm{Sr} /{ }^{86} \mathrm{Sr}$ values range from 0.67995 to 0.85226 , which is beyond the range of normal continental crust and primitive mantle. Thus, these data cannot be used to trace the source of magma due to the hydrothermal alteration during the $\mathrm{W}$ mineralization process.

Conversely, the activities of $\mathrm{Sm}$ and $\mathrm{Nd}$ and the relevant isotopic composition remain unchanged in the evolution and alteration process. The Sm-Nd isotopic composition could be considered as a reasonable indicator for the source region. In this research, $\varepsilon_{\mathrm{Nd}}(\mathrm{t})$ values of granitoids from the Chuankou ore field are -10.77 for $\mathrm{G}-1,-7.74$ to -9.3 for $\mathrm{G}-2$, and -6.53 to -10.07 for G-3. The samples plot in the Cathaysia basement field in the T(Ma) vs. $\varepsilon_{N d}(t)$ diagram (Figure $\left.15 b\right)$. The calculated $T_{D M 2}$ and $\varepsilon_{N d}(t)$ values (2090 Ma for G-1, 1684 to 1764 Ma for G-2, and 1471 to 1669 Ma for G-3) reveal a crustal origin by partial 
melting. G-1 was derived from the metamorphic basement in the Paleoproterozoic Era, while G-2 and G-3 were of homogeneous origin in the Mesoproterozoic Era. Significantly negative correlations of the formation ages with $\mathrm{T}_{\mathrm{DM} 2}(2090 \mathrm{Ma} \rightarrow 1684$ to $1764 \mathrm{Ma} \rightarrow$ 1471 to $1669 \mathrm{Ma})$ and $\varepsilon_{\mathrm{Nd}}(\mathrm{t})(-10.77 \rightarrow-9.3$ to $-7.74 \rightarrow-10.07$ to -6.53$)$ indicate that the proportion of crustal components in the source area decreased gradually; however, the composition of the mantle shows an obvious increasing trend. In the AMF vs. CMF diagram, the granitoids plot near the region of metapelitic sources and metagraywackes far from the metamorphic basalt and tonalite field. This indicates that the source rocks of granitoids from the Chuankou ore field are mainly crystal schists and gneisses formed by metamorphic Proterozoic mudstones and metagraywackes (Figure 15a).

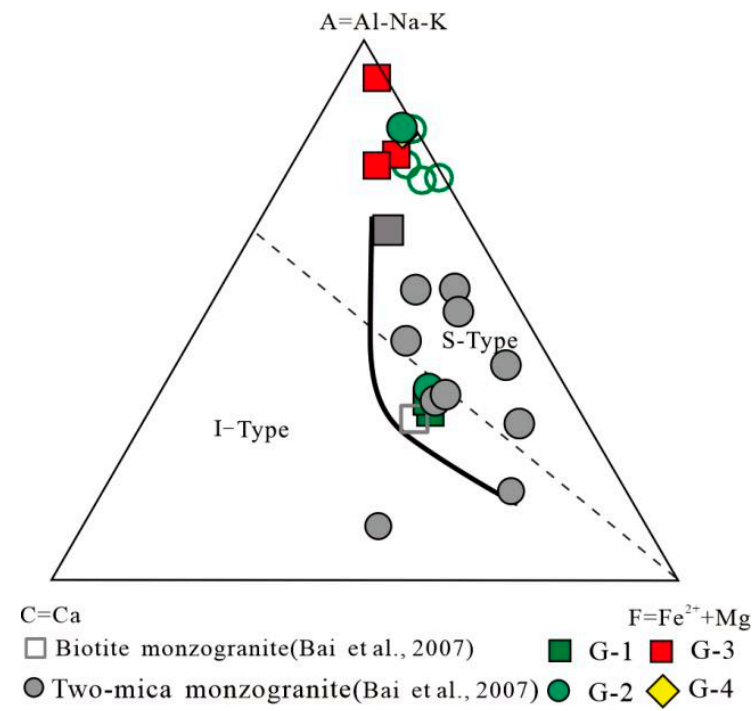

Figure 14. ACF diagrams of granitoids from Chuankou ore field.
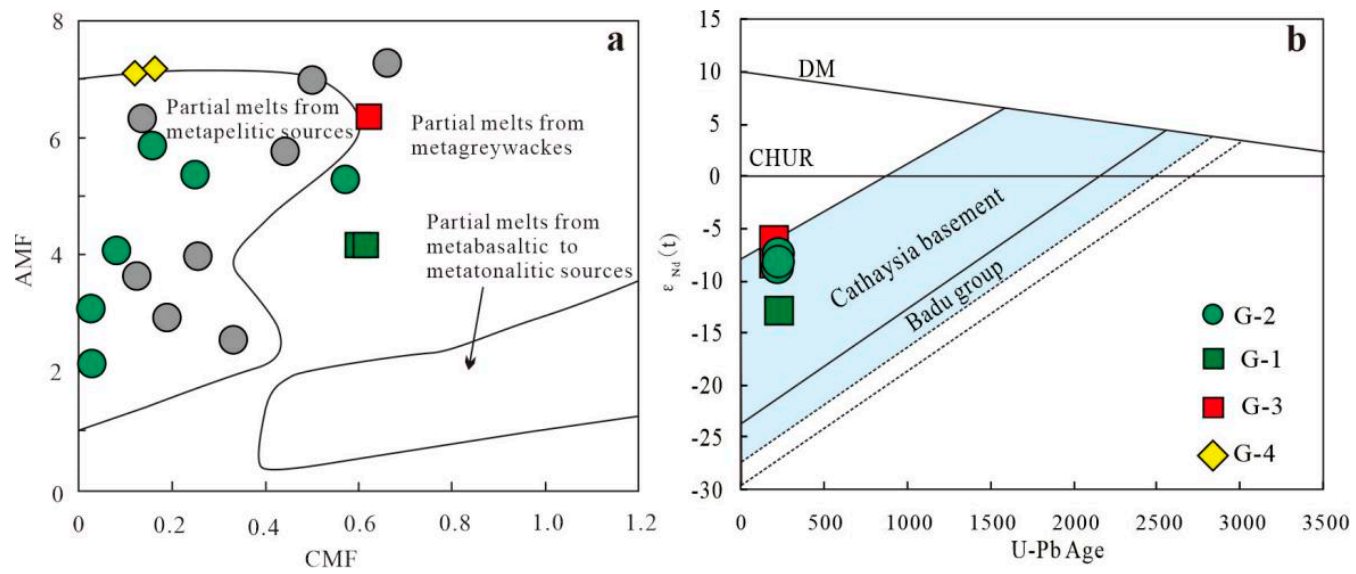

Figure 15. (a) AMF versus CMF diagrams, (b) $\varepsilon_{\mathrm{Nd}}(\mathrm{t})$ versus formation age diagrams.

\subsubsection{Magmatic Process}

During the granitic magmatism process, Ti was mainly absorbed in ilmenite, rutile, titanite, biotite and anatase. The separation of Ti-bearing phases at relatively moderate to low temperatures would have led to a significant depletion of $\mathrm{Ti}, \mathrm{Nb}, \mathrm{Ta}$. $\mathrm{Eu}, \mathrm{Sr}$, and $\mathrm{Ba}$ which existed stably by substituting into the $\mathrm{K}^{+}$site in the $\mathrm{K}$-feldspar and/or $\mathrm{Ca}^{2+}$ site in plagioclase. $\mathrm{P}$ is the dominant component of apatite. There is significant depletion of $\mathrm{Sr}, \mathrm{Ba}$, $\mathrm{P}$, and Ti of granitoids from the Chuankou granitoids, indicating obvious fractional crystallization of feldspar, biotite, Ti-bearing minerals, and apatite in magmatic processes [59]. In addition, the $\mathrm{Eu} / \mathrm{Eu}^{*}$ ratios, $\mathrm{Rb} / \mathrm{Sr}$ ratios, $\mathrm{Sr}$, and Ba could be used as markers to identify 
fractional crystallization. The correlations between $\mathrm{Rb} / \mathrm{Sr}$ and $\mathrm{Sr}$, Ba and $\mathrm{Sr}$, and $\mathrm{Eu} / \mathrm{Eu}^{*}$ and Ba suggest that the fractional crystallization of $\mathrm{K}$-feldspar, plagioclase and biotite was the main genetic mechanism (Figure 16a-d). For the REEs (La and $\mathrm{Yb}$ ), carrier minerals included zircon, apatite, allanite, and monazite. The correlations between between La and $\mathrm{La} / \mathrm{Yb}$ suggests that the melt was constrained by the fractional crystallization of allanite and monazite (Figure 16e). In addition, there are no obvious xenoliths (metamorphic slate in the Proterozoic) near the stratigraphic contact belt and no significant correlation between $\mathrm{SiO}_{2}$ content and $\varepsilon_{\mathrm{Nd}}(\mathrm{t})$ values. This implies that the fractional crystallization process was relatively clear for the felsic melt rather than for the extensive assimilation-fractional crystallization (AFC) process (Figure 16f).
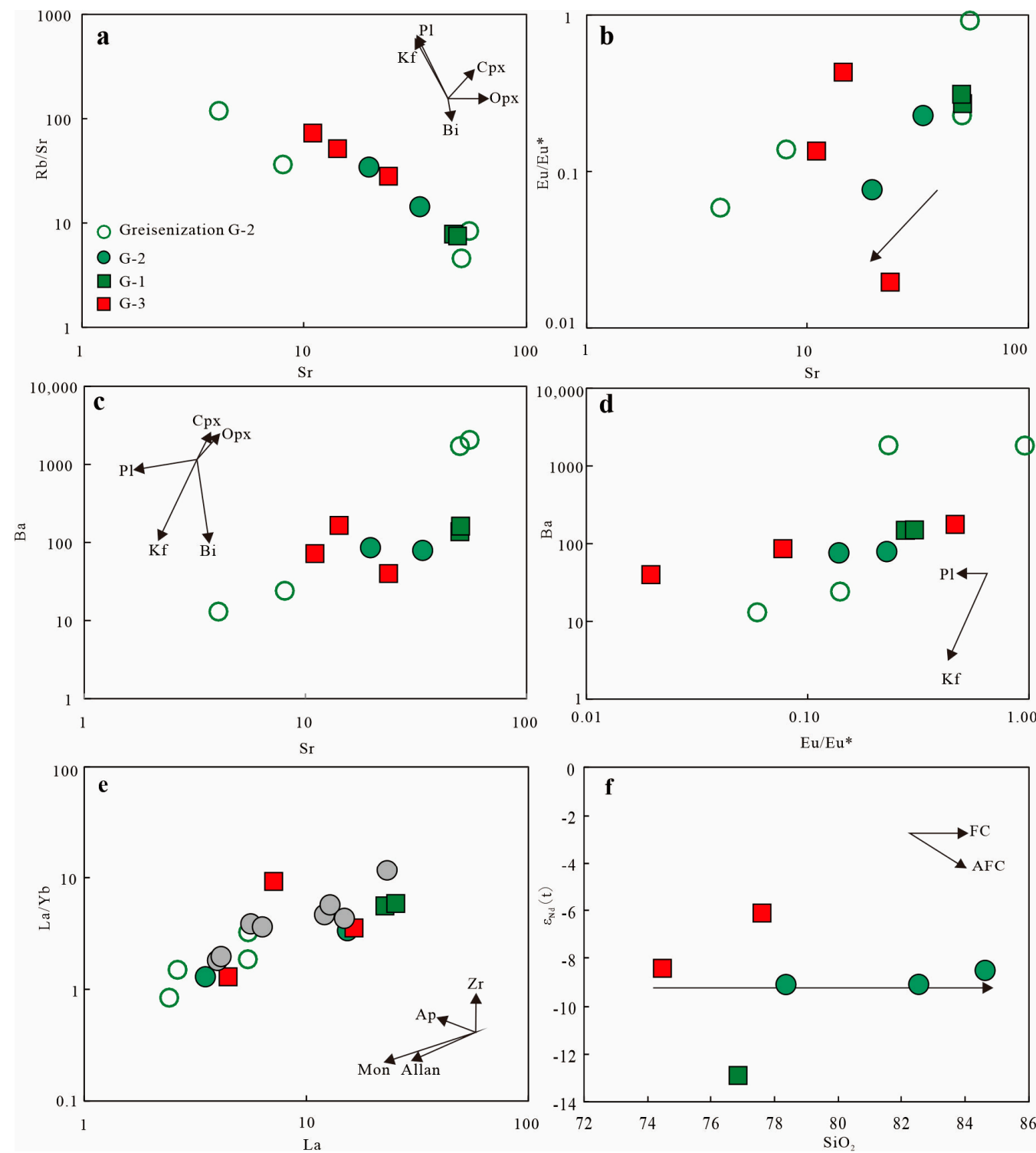

Figure 16. (a) $\mathrm{Rb} / \mathrm{Sr}$ versus Sr diagram, (b) Eu/Eu* versus Sr diagram, (c) Ba versus Sr diagram, (d) $\mathrm{Ba}$ versus $\mathrm{Eu} / \mathrm{Eu}^{*}$ diagram, (e) $\mathrm{La} / \mathrm{Yb}$ versus La diagrams, (f) $\varepsilon_{\mathrm{Nd}}(\mathrm{t})$ versus $\mathrm{SiO}_{2}$ diagram; $\mathrm{Zr}$ zircon, Ap- apatite, Mon- monazite, Allan- allanite, Opx- orthopyroxene, Cpx- clinopyroxene, Kf K-felspar, Pl- plagioclase, Bi- biotite, FC- fractional crystallization, AFC- assimilation.

Furthermore, $\mathrm{Zr}+\mathrm{Nb}+\mathrm{Y}$ contents of the Chuankou complex vary from 75.57 to $187.05 \mathrm{ppm}$, and the $\mathrm{Rb} / \mathrm{Ba}$ ratios range from 1.33 to 39.41. An obvious negative correlation trend is exhibited on the $\mathrm{Zr}+\mathrm{Nb}+\mathrm{Y}$ versus $\mathrm{Rb} / \mathrm{Ba}$ diagram, coinciding with the 
Sandy Cope granite field, indicating the common regulations of highly fractionated granite (Figure 17).

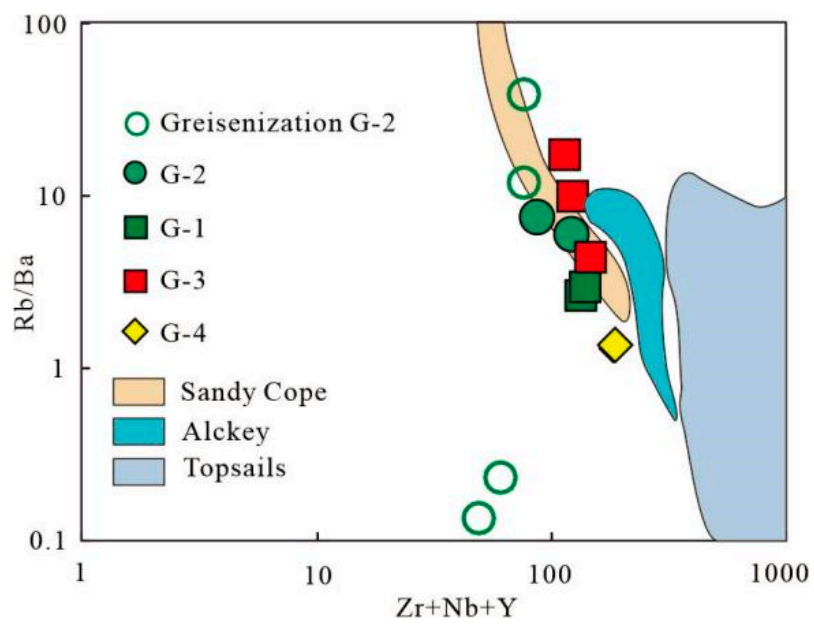

Figure 17. $\mathrm{Rb} / \mathrm{Ba}$ versus $\mathrm{Zr}+\mathrm{Nd}+\mathrm{Y}$ diagrams of intrusions in Chuankou Tungsten ore field, Sandy Cope and Alckey granite represent highly fractionated granite after [58].

\subsubsection{Relationships between Host Rocks and Tungsten Mineralization}

There are three main substitution mechanisms of scheelite in the concentration of REEs: (1) $2 \mathrm{Ca}^{2+} \leftrightarrow \mathrm{Na}^{+}+\mathrm{REE}^{3+}$, (2) $\mathrm{Ca}^{2+}+\mathrm{W}^{6+} \leftrightarrow \mathrm{REE}^{3+}+\mathrm{Nb}^{5+}$, and (3) $3 \mathrm{Ca}^{2+} \leftrightarrow 2 \mathrm{REE}^{3+}$ $+\square$ ( $\square$ vacancy) [60,61]. A significant comparative study between REE patterns of G-1/G-2 from the Chuankou ore field and Sch-3 was performed and showed high correlation [54]. In addition, the $\mathrm{Sr}$ isotopic composition $\left(\mathrm{I}_{\mathrm{sr}}\right)$ of $\mathrm{G}-1$ (0.72109) is close to the medium composition of Sch-1 and Sch-3, which is derived from magmatic-hydrothermal conditions without significant fluid/rock interactions and fluid mixing. In addition, G-1, G-2, and G-3 are highly fractionated S-type granite and contain $\mathrm{W}$ concentrations that are several to ten times higher than average crustal concentrations (1.9 ppm and $0.6 \mathrm{ppm}$, respectively [62]). This characteristic is very similar to the host rocks of well-known Dahutang superlarge $W$ deposits [63].

To date, the Chuankou $\mathrm{W}$ deposit has been identified as the largest Indosinian $\mathrm{W}$ deposit in the SCB and contains quartz vein type-, veinlet type-, and altered granite type-W ore bodies. Cai et al. obtained a formation age of $224.6 \pm 1.31 \mathrm{Ma}$ for the altered two mica monzogranites [57], which are generally thought to be host rocks of disseminated wolframite and scheelite. The ore formation ages of quartz vein-type mineralization ranged from 224 to $230 \mathrm{Ma}[31,56,64]$. These data are consistent with the ${ }^{206} \mathrm{~Pb} /{ }^{238} \mathrm{U}$ ages of G-1 $(230.8 \pm 1.6 \mathrm{Ma})$ and G-2 (222-224 Ma). Field observations have also shown the close spatiotemporal relationship between G-1, G-2, and W mineralization. However, the ages of G-3 and G-4 are 203.1 $\pm 1.6 \mathrm{Ma}$ and $135.5 \pm 2.4 \mathrm{Ma}(\mathrm{MSWD}=1.3)$, respectively. Seemingly, these intrusions were emplaced after $\mathrm{W}$ mineralization.

Systematic evidence indicates that the host rocks of the Chuankou W ore field were G-1 and G-2. However, how did W separate from the intrusions and become vastly concentrated in a limited spatial area? Generally, rutile was the main W-bearing mineral during the early stage of magmatic activity, while wolframite and scheelite dominated the later stage of magmatic to hydrothermal activity. Because the six-coordination $\mathrm{Ti}^{4+}$ could be substituted by $\mathrm{W}^{6+}$ accompanied by a double substitution of Fe to maintain the charge balance [65], $\mathrm{W}$ could be concentrated in large amounts in rutile and was significantly depleted in the residual melt and fluid. However, the granitoids from the Chuankou ore field (G-1 and G-2) contain $0.26-0.35$ wt. $\% \mathrm{MgO}$ and 1.29-1.77 wt.\% $\mathrm{FeO}^{\mathrm{T}}$ and belong to the normal ilmenite-series granite, indicating an obvious absence of rutile in the early crystalline phase $[63,66]$. In addition, $W$ is a lithophilic element in the bulk silicon earth (BSE), and the multiple stages of partial melting and separation crystallization would have caused a 
strong concentration of $\mathrm{W}$ in the late period of the residual melt phase. Thus, G-1 and G-2 granitoids have significant potential for the mineralization of $\mathrm{W}$.

In addition, with increasing oxygen fugacity, the mineralization series of $\mathrm{Sn} \rightarrow \mathrm{W}$ $\rightarrow \mathrm{Mo} \rightarrow \mathrm{Cu}(\mathrm{Mo}) \rightarrow \mathrm{Cu}(\mathrm{Au})$ was carried out in succession [67]. The occurrence of $\mathrm{W}$ mineralization could be attributed to the reduced granitic magmas that typically belong to the ilmenite series [68,69]. A possible contribution from $\mathrm{W}^{4+}$ may have only been at the very lowest oxygen fugacity accessible to the experimental method in the melt [70-72]. Zircon is a common accessory mineral in intermediate-acid igneous rocks and is stable during later hydrothermal alteration and physiochemical processes. Due to its similar ionic radii and electrovalence, $\mathrm{Ce}^{4+}$ is more easily absorbed in zircon crystals than light rare earth metal ions (such as $\mathrm{Ce}^{3+}$ ) that occupy the site of $\mathrm{Zr}^{4+}$ under oxidizing conditions. Hence, zircon can be invoked as a tracer for the evaluation of relative oxygen fugacity based on its $\mathrm{Ce}^{4+} / \mathrm{Ce}^{3+}$ ratios. In this paper, the value of $\mathrm{Ce}^{4+} / \mathrm{Ce}^{3+}$ was calculated as 0.33-93.28, which is much lower than the host rocks of well-known, large-scale, porphyry $\mathrm{Cu}-\mathrm{Au}$ deposits, such as Chuquicamata-El Abra [50], and typical $\mathrm{Cu}-\mathrm{Au}$ (Mo) deposits from the SCB, such as Dabaoshan porphyry Mo deposits $\left(\mathrm{Ce}^{4+} / \mathrm{Ce}^{3+}=356-1300\right.$; Li et al. $)$ [73] and Dexin porphyry $\mathrm{Cu}$ deposits $\left(\mathrm{Ce}^{4+} / \mathrm{Ce}^{3+}=495-1922\right)$ [53]. In contrast, the $\mathrm{Ce}^{4+} / \mathrm{Ce}^{3+}$ ratios were closer to those of W and Sn-bearing granitoids, such as the Guposhan, Qitianling, and Xuehuading granitoids, suggesting a significant metallogenetic potential of W and Sn [69] (Figure 18).

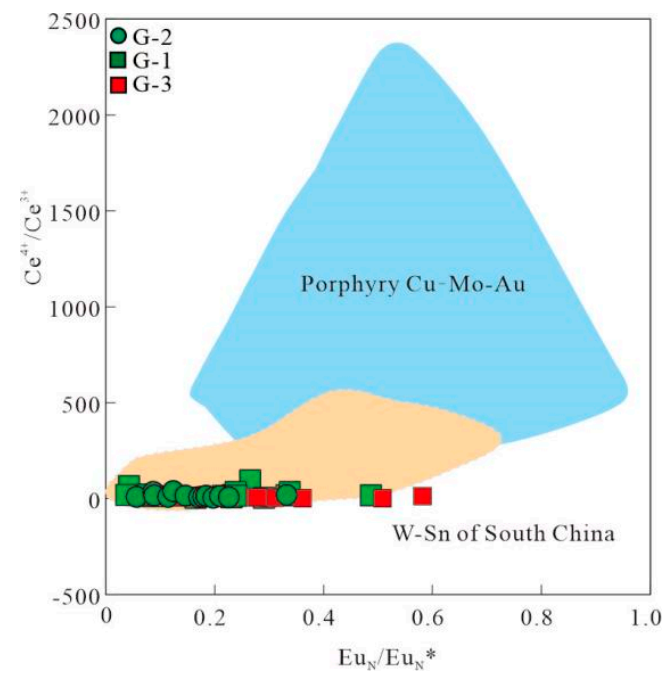

Figure 18. The $\mathrm{Ce}^{4+} / \mathrm{Ce}^{3+}$ versus $\mathrm{Eu}_{\mathrm{N}} / \mathrm{Eu}_{\mathrm{N}}{ }^{*}$ diagram. The data of blue field named Porphyry $\mathrm{Cu}-\mathrm{Mo}-\mathrm{Au}$ are from $[49,70]$ and there in, the orange field are from [74].

Blevin [75] carried out important work on the granite in the Lachlan fold belt and proposed the parameters to estimate the redox state of granite [75]:

$$
\begin{gathered}
\Delta \mathrm{Ox} 1=\mathrm{Fe}_{2} \mathrm{O}_{3} / \mathrm{FeO}(\text { wt. } \%) \\
\Delta \mathrm{O} \times 2=\log \left(\mathrm{Fe}_{2} \mathrm{O}_{3} / \mathrm{FeO}\right)+0.3+0.03 \mathrm{FeO}^{\mathrm{T}}(\text { wt. } \%)
\end{gathered}
$$

The calculated results show that the redox state $(\Delta \mathrm{O} \times 1)$ of $\mathrm{G}-1$ ranges from 0.03 to 0.31 , that of G-2 ranges from 0.09 to 0.91 , that of G-3 ranges from 0.41 to 1.68 , and that of $\mathrm{G}-4$ is 0.35 . The $\Delta \mathrm{Ox} 2$ of $\mathrm{G}-1$ ranges from -1.19 to -0.16 , that of $\mathrm{G}-2$ ranges from -0.70 to 0.32 , that of G-3 ranges from -0.06 to 0.56 , and that of G-4 is -0.13 . Obviously, G- 1 and most G-2 had the lowest degree of oxidation. This condition provides an opportunity to remove substantial $W$ from magma to hydrothermal fluids. Indeed, the slightly higher values of $\Delta \mathrm{O} \times 1$ and $\Delta \mathrm{O} \times 2$ in G-3 and G-4 indicate that $\mathrm{W}$ would have remained in biotite or muscovite by substitution with the $\mathrm{Al}^{3+}$ and/or $\mathrm{Ga}^{3+}$ site instead of expulsion from the 
melt. Further investigation is needed for the relationship between G-3, G-4 granitoids, and regional $\mathrm{W}$ mineralization.

\subsection{Metallogenesis and Geodynamic Implications}

During the early Middle Triassic, the intense collision and extensive metamorphism between the Indo-China block and Sibumas-Qingtang block exerted far-reaching effects on the SCB [76,77]. In addition, the southeastward subduction and collision of the North China block (NCB) with the South China block (SCB) overlapped due to the closure of the Paleo-Tethys Ocean. The SCB experienced multidirectional compression and extensive shortening, accompanied by thickening of the continental lithosphere [78-82]. During the late Mesozoic period, due to the tectonic regime transformation from Paleotethys dominant to paleo-Pacific tectonic dominant, the tectonic axis changed from the E-W direction to the NE-SW direction $[40,83]$. The tectonic regime is characterized by multiple stages of compression and extension, resulting in the formation of extensive magmatism and mineralization $[9,39,84-86]$.

Indosinian $\mathrm{W}$ deposits are zonal and near the E-W direction, whereas Yanshanian $\mathrm{W}$ deposits are distributed in the NE-SW direction. The formation age of Indosinian W-Sn deposits in the SCB reveals that the two stages of W mineralization formed from 231.4 to $225 \mathrm{Ma}$ and 213.3 to $193 \mathrm{Ma}$ [56]. Two peak values of age data from Indosinian igneous rocks have been proposed [87-89]; the early stage aged from 243 to $233 \mathrm{Ma}$, while the late stage is from 222 to $204 \mathrm{Ma}$. There is a strong coupling relationship between Indosinian W deposits and igneous rocks. In addition, the $\mathrm{W}$ deposits in Guangxi Province vary from 214.1 to 211.9 Ma, which is reasonably linked to Miao'ershan and Limu granites (western part). The W deposits in Hunan Province and Jiangxi Province were formed from 230 to 225.4 Ma and 231.4 to $202 \mathrm{Ma}$, respectively (central part). The W deposits of Yunnan and Fujian Provinces are significantly younger than those from the central part of SC, which formed from 209 to $207 \mathrm{Ma}$ and 226 to $193 \mathrm{Ma}$ (eastern part) (Figure 1b). A possible "V"-shaped distribution model in the region indicates that the central belts of $W$ deposits are relatively older than the others. The western and eastern parts have significantly lower values than those in the central part, which may represent the reactivation of the Proterozoic Qin-Hang tectonic belt under the Indosinian collision orogenetic regime of SC.

Regional Sr-Nd isotopic compositions show that $\varepsilon_{\mathrm{Nd}}(\mathrm{t})$ values of Indosinian granitoids range from -14.4 to $-8[17,90]$. The two-stage depleted mantle model ages of Indosinian granitoids range from 1.63 to $2.09[17,90]$. In general, the $\mathrm{T}_{\mathrm{DM} 2}$ values better match the formation ages of the Paleoproterozoic metamorphic basement of the SCB [82]. On the other hand, Yanshanian $\mathrm{T}_{\mathrm{DM} 2}$ values range from 1.04 to 2.28, especially in Northeast Jiangxi. The Nanling area and coastal zone of Fujian and Zhejiang Provinces show multiple belts of low $\mathrm{T}_{\mathrm{DM}}$ values $(<1.6 \mathrm{Ga})$ and high $\varepsilon_{\mathrm{Nd}}(\mathrm{t})$ values $(>-9)$, which might match the Mesoproterozoic basement [38,91-93]. Numerous research data confirm that the main source of Yanshanian W mineralization was the Mesoproterozoic metamorphic basement, such as the Shuangqiaoshan group [81,94,95], which has an abnormal enrichment of W content-ten times more than the concentration of the average crust (11.7 ppm of the Shuangqiaoshan group) [96]. The more ancient basement identified in this study suggests a relatively deeper derivation of Indosinian $W$ mineralization. Many valuable insights have been reported regarding the tectonic mechanism of $W$ mineralization in the SCB, and the consensus suggests that the large Yanshanian W mineralization in the SCB was constrained closely by the paleo-Pacific plate regime, which mainly includes the extension of the ShiHang belt [38], a mantle plume [7,97], back-arc extension and lithospheric thinning [98], and slab subduction [99,100]. However, a distinct dynamic mechanism was identified in which Indosinian magmatism and mineralization extended approximately east-west in a zone that formed under the extension of a post-collisional setting, which could have been linked to the closure effects of the ancient Tethys Ocean. This setting reflects a relative "free" extension space of the overall compression regime $[40,101]$. 
Studies have recently revealed two dominant mineral assemblages and two stages of tectonic regimes in the Indosinian in SC [48,95]. G-1, G-2, and G-3 formed about 20-10 Ma later than the peak period of orogeny triggered by the collage of the SCB, North China craton, and Indo-China block. This reflects a post-collisional setting, which is parallel to the contemporaneous A-type granite in the SCB. In the late stage of the magmatic processes of G-1 and G-2, fertile magmatic fluid converged on the upper part of the granitoids and filled the internal fissure of the slate with the formation of extensive greisenization and granite-type wolframite (Maowan, Wubeichong, and Baishui) and quartz vein-type wolframite (Huanglong, Nanwan, and Sanjiaotan) interior contact belt. The continuous migration of ore-forming fluid up to the interbedded limestone and shale of the Devonian Yanglinao formation occurred $\left(\mathrm{D}_{2} \mathrm{y}\right)$. Adequate fluid-rock interactions and abundant $\mathrm{Ca}^{2+}$ ion reservoirs from the strata made it possible for large-scale dissemination and veinlet scheelite to form (Figure 19b).

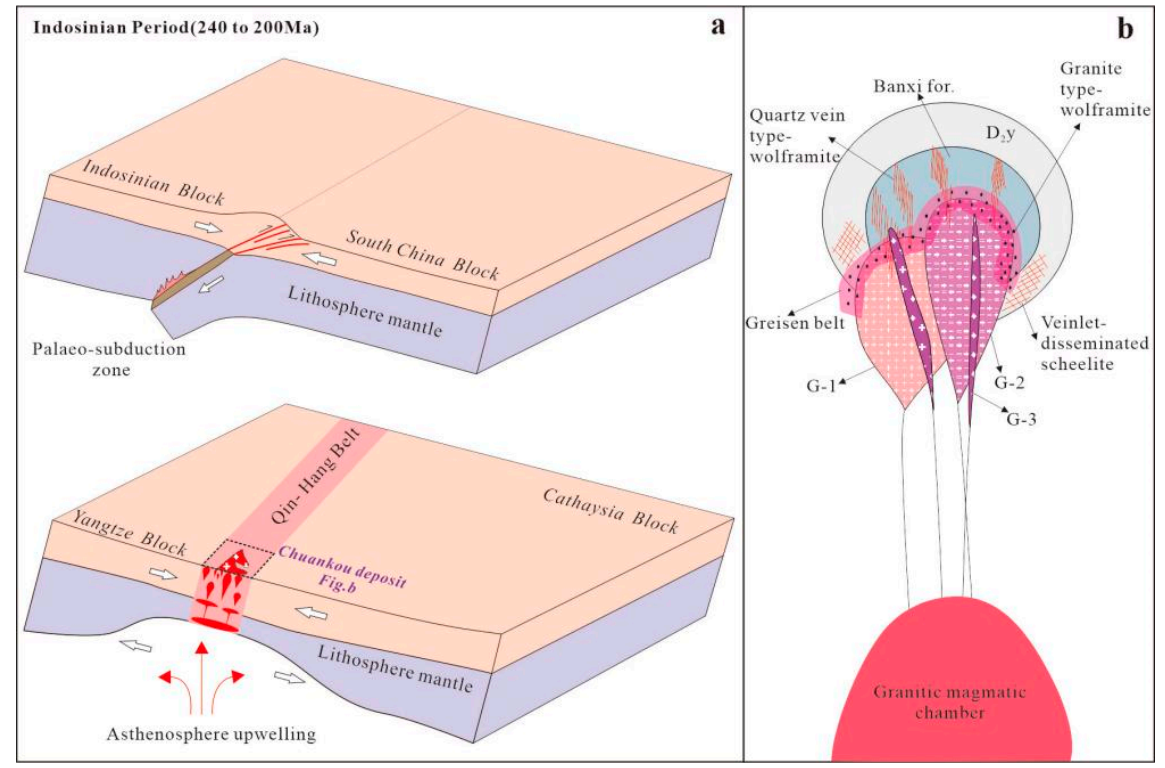

Figure 19. (a) Model explaining the geodynamic setting for the Indosinian W mineralization in SC and (b) Model explaining the formation of the granitoids and their relationship to tungsten mineralization.

\section{Conclusions}

(1) The formation age of G-1 is $230.8 \pm 1.6 \mathrm{Ma}(\mathrm{MSWD}=0.31), \mathrm{G}-2$ is $222.1 \pm 0.56 \mathrm{Ma}$, G-3 is $203.1 \pm 1.6 \mathrm{Ma}(\mathrm{MSWD}=7.2)$, and G-4 is $135.5 \pm 2.4 \mathrm{Ma}(\mathrm{MSWD}=1.3)$. The emplacement sequence is G-1 (phase I), G-2 (phase II), G-3 (phase III), and G-4 (phase IV).

(2) Granitoids from the Chuankou ore field had significantly high contents of Si and Al and low contents of alkali, $\mathrm{Fe}, \mathrm{Mg}, \mathrm{Mn}$, and $\mathrm{Ca}$. The granites are commonly enriched in $\mathrm{Rb}$, $\mathrm{Zr}, \mathrm{Hf}, \mathrm{Th}$, and $\mathrm{U}$ but depleted in $\mathrm{Ba}, \mathrm{Sr}, \mathrm{P}$, and Ti, indicating obvious highly fractionated S-type granite affinities. The Chuankou complex was derived from the partial melting of the Cathaysia basement and underwent significant fractionation of K-feldspar, plagioclase, biotite, Ti-bearing minerals (except rutile), zircon, apatite, allanite, and monazite.

(3) G-1 and G-2 showed a more reductive state than G-3 and even typical host rocks of porphyry copper deposits were identified to have an obvious correlation with $\mathrm{W}$ mineralization of the Chuankoou ore field.

(4) Indosinian $\mathrm{W}$ deposits were formed in a post-collision setting triggered by the collisional orogeny of SC in the late Paleozoic to early Mesozoic. However, the Yanshanian W deposits reflect strengthened crust-mantle interactions which resulted from the multistage extension of the SCB caused by the westward subduction of the paleo-Pacific plate. 
Author Contributions: Conceptualization, J.Q. and D.W.; methodology, J.Q.; software, J.Q.; validation, D. and Y.C.; formal analysis, J.Q.; investigation, J.Q. and D.W.; resources, J.Q.; data curation, D.W.; writing—original draft preparation, J.Q.; writing—review and editing, J.Q. and D.W.; visualization, J.Q.; supervision, D.W.; project administration, D.W.; funding acquisition, D.W. All authors have read and agreed to the published version of the manuscript.

Funding: This paper is financed by the China Geological Survey's projects (DD20190397) and National Natural Science Foundation of China (NSFC 41802082).

Acknowledgments: This paper is financed by the China Geological Survey's projects (DD20190397) and National Natural Science Foundation of China (NSFC 41802082). We would like to thank Qin Jin-nin, Cai Fu-cheng, Liu Shan-bao and Wang Cheng-hui, Fan Ming-feng and other geologists for their help during the field investigations and samples analysis.

Conflicts of Interest: The authors declare no conflict of interest.

\section{References}

1. Chen, Z.G.; Zhang, L.C.; Lu, B.Z.; Li, Z.L.; Wu, H.Y.; Xiang, P.; Huang, S.W. Geochronology and geochemistry of the Taipingchuan copper-molybdenum deposit in Inner Mongolia, and its geological significances. Acta Petrol. Sin. 2010, 26, 1437-1449, (In Chinese with English abstract). [CrossRef]

2. Mao, J.W.; Cheng, Y.B.; Chen, M.H.; Pirajno, F. Major types and time-space distribution of mesozoic ore deposits in South China and their geodynamic settings. Miner. Depos. 2013, 48, 267-294.

3. USGS (U.S. Geological Survey). Mineral Commodity Summaries; U.S. Government Printing Office: Washington, DC, USA, 2009; pp. 1-179.

4. Hsu, K.C. Tungsten deposits of southern Kiangsi, China. Econ. Geo. 1943, 138, 431-474. [CrossRef]

5. Lu, H.Z. Origin of Tungsten Mineral Deposit in South China; Publishing House of Chongqing: Chongqing, China, 1986; pp. 1-232, (In Chinese with English abstract).

6. Mao, J.W.; Li, H.Y.; Pei, R.F. Nd-Sr isotopic and petrogenetic studies of the Qianlishan granite stock, Hunan Province. Miner. Depos. 1995, 14, 235-242, (In Chinese with English abstract).

7. Mao, J.W.; Li, H.Y.; Song, X.X.; Rui, B.; Xu, Y.Z.; Wang, D.H.; Lan, X.M.; Zhang, J.K. Geology and Geochemistry of the Shizhuyuan W-Sn-Mo-Bi Polymetallic Deposit, Hunan Province; Geological Publishing House: Beijing, China, 1998; pp. 1-215. (In Chinese)

8. Mao, Z.H.; Cheng, Y.B.; Liu, J.J.; Yuan, S.D.; Wu, S.H.; Xiang, X.K.; Luo, X.H. Geology and molybdenite Re-Os age of the Dahutang granite-related veinlets-disseminated tungsten ore field in the Jiangxin Province, China. Ore Geol. Rev. 2013, 53, 422-433. [CrossRef]

9. Li, Z.X.; Evans, D.A.D.; Zhang, S. A $90^{\circ}$ Spin on Rodinia: Possible causal links between the Neoproterozoic supercontinent, superplume, True polar wander and low-latitude glaciation. Earth Planet. Sci. Lett. 2004, 220, 409-421. [CrossRef]

10. Peng, J.; Zhou, M.F.; Hu, R.; Shen, N.; Yuan, S.; Bi, X. Precise molybdenite Re-Os and mica Ar-Ar dating of the Mesozoic Yaogangxian tungsten deposit, central Nanling district, South China. Miner. Depos. 2006, 41, 661-669. [CrossRef]

11. Yuan, S.; Peng, J.; Hu, R.; Li, H.; Shen, N.; Zhang, D. A precise U-Pb age on cassiterite from the Xianghualing Tin-polymetallic deposit (Hunan, South China). Miner. Depos. 2008, 43, 375-382. [CrossRef]

12. Liu, Y.; Hu, Z.; Gao, S.; Günther, D.; Xu, J.; Gao, C. In situ, analysis of major and trace elements of anhydrous minerals by LA-ICP-MS without applying an internal standard. Chem. Geol. 2008, 257, 34-43. [CrossRef]

13. Wang, D.H.; Chen, Z.H.; Chen, Y.C.; Tang, J.X.; Li, J.K.; Ying, L.J.; Wang, C.H.; Liu, S.B.; Li, L.X.; Qin, Y.; et al. New data of the rock forming and ore-forming chronology for China's important mineral resources areas. Acta Geol. Sin. 2010, 84, 1030-1040, (In Chinese with English abstract).

14. Feng, C.; Zeng, Z.; Zhang, D.; Qu, W.; Du, A.; Li, D.; She, H. SHRIMP zircon U-Pb and molybdenite Re-Os isotopic dating of the tungsten deposits in the Tianmenshan-Hongtaoling W-Sn orefield, southern Jiangxi province, China, and geological implications. Ore Geol. Rev. 2011, 43, 8-25. [CrossRef]

15. Feng, C.; Zheng, Z.; Qu, W.; Zeng, Z. Temporal consistency between granite evolution and tungsten mineralization in Huamei'ao, southern Jiangxi province, China, evidence from precise zircon $\mathrm{U}-\mathrm{Pb}$, molybdenite Re-Os, and muscovite ${ }^{40} \mathrm{Ar}-{ }^{39} \mathrm{Ar}$ isotope geochronology. Ore Geol. Rev. 2015, 65, 1005-1020. [CrossRef]

16. Huang, J.C.; Peng, J.T.; Yang, J.H.; Zhang, B.L.; Xu, C.X. Precise zircon U-Pb and molybdenite Re-Os dating of the Shuikoushan granodiorite-related $\mathrm{Pb}-\mathrm{Zn}$ mineralization, southern Hunan, South China. Ore Geol. Rev. 2015, 71, 305-317. [CrossRef]

17. Guo, C.L.; Lv, X.Q.; Lou, F.S.; Zeng, Z.L. Petrography, genetic types and geological dynamical settings of the Indosinian granitoids in South China. Geotecton. Metallog. 2012, 363, 457-472, (In Chinese with English abstract).

18. Chen, P.R. Geodynamic setting of Mesozoic magmatism and its relationgship to uranium metallogenesis in southeastern China. Uranium Geol. 2004, 20, 266-270, (In Chinese with English abstract).

19. Zhao, K.D.; Jiang, S.Y.; Chen, W.F.; Chen, P.R.; Lin, H.F. Mineralogy, geochemistry and ore genesis of the Dawan uranium deposit in southern Hunan Province, South China. J. Geochem. Explor. 2014, 138, 59-71. [CrossRef] 
20. Ma, T.Q.; Bai, D.Y.; Kuang, J.; Wang, X.H. Zircon SHRIMP dating of the Xitian granite pluton, Chaling, south- its geological significance. Geol. Bull. China 2005, 24, 415-419, (In Chinese with English abstract).

21. Cai, M.H.; Chen, K.X.; Qu, W.J.; Liu, G.Q.; Fu, J.M.; Yin, J.P. Geological characteristics and Re-Os dating of molybdenites in Hehuaping tin_polymetallic deposit, southern Hunan Province. Miner. Depos. 2006, 25, 263-268, (In Chinese with English abstract).

22. Liang, H.Y.; Wu, J.; Sun, W.D.; Mo, J.H.; Huang, W.T. Discussion on mineralization of Indosinian in South China. J. Miner. 2011, 31 (Suppl. S1), 53-54, (In Chinese with English abstract).

23. Zhao, W.W.; Zhou, M.F.; Williams-Jones, A.E.; Zhao, Z. Constraints on the uptake of REE by scheelite in the Baoshan tungsten skarn deposit. South China. Chem. Geol. 2018, 477, 123-136. [CrossRef]

24. Zhao, Z.; Zhao, W.W.; Lu, L.; Wang, H.Y. Constraints of multiple dating of the Qingshan tungsten deposit on the Triassic W(-Sn) mineralization in the Nanling region, South China. Ore Geol. Rev. 2018, 94, 46-57. [CrossRef]

25. Yokart, B.; Barr, S.M.; Williams-Jones, A.E.; Macdonald, A.S. Late-stage alteration and tin-tungsten mineralization in the Khuntan Batholith, northern Thailand. J. Asian Earth Sci. 2003, 21, 999-1018. [CrossRef]

26. Breiter, K. Nearly contemporaneous evolution of the A-and S-type fractionated granites in the Krušné hory/Erzgebirge Mts., Central Europe. Lithos 2012, 151, 105-121. [CrossRef]

27. Zhang, Y.; Yang, J.H.; Chen, J.Y.; Wang, H.; Xiang, Y.X. Petrogenesis of Jurassic tungsten-bearing granites in the Nanling Range, South China: Evidence from whole-rock geochemistry and zircon U-Pb and Hf-O isotopes. Lithos 2017, 278-281, 166-180. [CrossRef]

28. Jiang, H.; Jiang, S.Y.; Li, W.Q.; Zhao, K.D.; Penga, N.J. Highly fractionated Jurassic I-type granites and related tungsten mineralization in the Shirenzhang deposit, northern Guangdong, South China: Evidence from cassiterite and zircon U-Pb ages, geochemistry and Sr-Nd-Pb-Hf isotopes. Lithos 2018, 312-313, 186-203. [CrossRef]

29. Huang, W.T.; Wu, J.; Zhang, J.; Laing, H.Y.; Qiu, X.L. Geochemistry and Hf-Nd isotope characteristics and forming processes of the Yuntoujie granites associated with W-Mo deposit, Guangxi, South China. Ore Geol. Rev. 2017, 81, 953-964. [CrossRef]

30. Bai, D.Y.; Huang, J.Z.; Liu, Y.R.; Wu, G.Y.; Ma, T.Q.; Wang, X.Y. Freamwork of Mesozoic tectonic evolution of southeastern Huanan and the Huanan-Guangdong-Jiangxi border area. China Geol. 2005, 33, 557-570, (In Chinese with English abstract).

31. Peng, N.L.; Wang, X.H.; Yang, J.; Chen, D.; Luo, L.; Luo, P.; Liu, T.Y. Re-Os dating of molybdenite from Sanjiaotan tungsten deposit in Chuankou area, Hunan Province, and its geological implication. Miner. Depos. 2017, 36, 1402-1414, (In Chinese with English abstract).

32. Mao, J.W.; Xie, G.Q.; Guo, C.L.; Chen, Y.C. Large-scale tungsten-tin mineralization in the Nanling region, South China: Metallogenic ages and corresponding geodynamic processes. Acta Petrol. Sin. 2007, 23, 2329-2338, (In Chinese with English abstract).

33. Yang, M.G.; Huang, S.B.; Lou, F.S.; Tang, W.X.; Mao, S.B. Lithospheric structure and large-scale metallogenic process in Southeast China continental area. Geol. China 2009, 36, 528-543, (In Chinese with English abstract).

34. Li, H.; Cao, J.; Algeo, T.J.; Jiang, W.; Wu, Q. Zircons reveal multi-stage genesis of the Xiangdong (Dengfuxian) tungsten deposit, South China. Ore Geol. Rev. 2019, 111, 102979. [CrossRef]

35. Shui, T.; Xu, B.T.; Liang, R.H.; Qiu, Y.S. Shaoxing-Jiangshan ancient land docking zone. Sci. Bull. 1986, 31, 444-448, (In Chinese with English abstract).

36. Greentree, M.R.; Li, Z.X.; Li, X.H.; Wu, H. Late Mesoproterozoic to earliest Neoproterozoic basin record of the Sibao orogenesis in western South China and relationship to the assembly of Rodinia. Precambrian Res. 2006, 1511-1512, 79-100. [CrossRef]

37. Li, X.H.; Li, Z.X.; Zhou, H.W.; Liu, Y.; Kinny, P.D. U-Pb zircon geochronology, geochemistry and Nd isotopic study of neoproterozoic bimodal volcanic rocks in the Kangdian rift of South China, implications for the initial rifting of rodinia. Precambrian Res. 2002, 113, 135-154. [CrossRef]

38. Gilder, S.A.; Gill, J.; Coe, R.S.; Zhao, X.; Liu, Z.; Wang, G. Isotopic and paleomagnetic constraints on the mesozoic tectonic evolution of South China. J. Geophys. Res. Solid Earth 1996, 101, 16137-16154. [CrossRef]

39. Li, X.H. Cretaceous magmatism and lithospheric extension in southeast China. J. Asian Earth Sci. 2000, 183, 293-305. [CrossRef]

40. Shu, L.S. An analysis of principal features of tectonic evolution in South China Block. Geol. Bull. China 2012, 31, 1035-1053, (In Chinese with English abstract).

41. Deng, X.W. Analysis Districts Metallogenic Model about Xiaosanjiaotan Ore Chuankou Tungsten Orefield, Hunan Province; Central South University: Changsha, China, 2009; 76p, (In Chinese with English abstract).

42. Nasdala, L.; Irmer, G.; Wolf, D. The degree of metamictization in zircon, A raman spectroscopic study. Eur. J. Mineral. 1995, 7, 471-478. [CrossRef]

43. Sláma, J.; Košler, J.; Condon, D.J.; Crowley, J.L.; Gerdes, A.; Hanchar, J.M.; Horstwood, M.S.A.; Morris, G.A.; Nasdala, L.; Norberg, N.; et al. Plešovice zircon-a new natural reference material for U-Pb and Hf isotopic microanalysis. Chem. Geol. 2008, $249,1-35$. [CrossRef]

44. Ludwig, K.R. Isoplot/Ex, a Geochronological Toolkit for Microsoft Excel, Version 3.00; Berkeley Geochronology Center: Berkeley, CA, USA, 2003.

45. JY/T 015-1996. General Rules for Inductively Coupled Plasma-Atomic Emission Spectrometry (In Chinese). Available online: https:/ / www.chinesestandard.net/PDF/English.aspx/JYT015-1996 (accessed on 1 November 2021).

46. Xiao, Y.K.; Wei, H.Z.; Wang, Q.Z.; Zhang, C.G.; Sun, A.D. A devices for measuring low temperature of filamentin ion source of VG-354 thermal ionization mass spectrometer and its application. Chin. J. Anal. Chem. 2002, 30, 1272-1276, (In Chinese with English abstract). 
47. Zhang, H.F.; Sun, M.; Zhou, X.H.; Fan, W.M.; Zhai, M.G.; Yin, J.F. Mesozoic lithosphere destruction beneath the North China Craton, evidence from major-, trace-element and $\mathrm{Sr}-\mathrm{Nd}-\mathrm{Pb}$ isotope studies of Fangcheng basalts. Contrib. Mineral. Petrol. 2002, 144, 241-254. [CrossRef]

48. Sun, S.S.; McDonough, W.F. Chemical and isotopic systematics of oceanic basalts: Implications for mantle 682 composition and processes. Geological Society of London. Spec. Publ. 1989, 42, 313-345. [CrossRef]

49. Hoskin, P.W.O.; Schaltegger, U. The composition of zircon and igneous and metamorphic petrogenesis. Rev. Mineral. Geochem. 2003, 53, 27-62. [CrossRef]

50. El-Bialy, M.Z.; Ali, K.A. Zircon trace element geochemical constraints on the evolution of the Ediacaran (600-614Ma) post collisional Dokhan volcanic and Younger granites of SE Sinai, NE Arabian-Nubian Shield. Chem. Geol. 2013, 361, 54-73. [CrossRef]

51. Fornelli, A.; Langone, A.; Micheletti, F.; Francesca, G. Ree partition among zircon, orthopyroxene, amphibole and garnet in a high-grade metabasic system. Geol. Mag. 2018, 155, 1705-1726. [CrossRef]

52. Ballard, R.J.; Palin, J.M.; Camplell, H.I. Relative oxidation states of magmas inferred from Ce(IV)/Ce(III) in zircon: Application to porphyry copper deposits of northern Chile. Contrib. Mineral. Petrol. 2002, 144, 347-364. [CrossRef]

53. Zhang, H.; Ling, M.X.; Liu, Y.L. High Oxygen Fugacity and Slab Melting Linked to Cu Mineralization: Evidence from Dexing Porphyry Copper Deposits, Southeastern China. J. Geol. 2013, 121, 289-305. [CrossRef]

54. Blundy, J.; Wood, B. Prediction of crystal-melt partition coefficients from elastic moduli. Nature 1994, 372, 452-454. [CrossRef]

55. Bai, D.Y.; Wang, Y.Q.; Wang, X.H.; Ma, T.Q.; Zhang, X.Y. Geochemistry, petrogenesis and tectonic setting of the early Yanshanian peraluminous granites in the Chuankou region, Hengyang, Hunan. Sediment. Geol. Tethyan Geol. 2007, 27, 49-59, (In Chinese with English abstract).

56. Qin, J.H.; Wang, D.H.; Li, C.; Chen, Y.C.; Cai, F.C. The molybdenite Re-Os isotope chronology, in situ scheelite and wolframite trace elements and $\mathrm{Sr}$ isotope characteristics of the Chuankou tungsten ore field, South China. Ore Geol. Rev. 2020, 126, 103756. [CrossRef]

57. Cai, F.C.; Qin, J.H.; Qin, J.N.; Jiang, B.G.; Zhu, C.S. The Geochemical Characteristics and LA-ICP-MS Zircon U-Pb Dating of Ore-bearing Granite of Chuankou intrusion-related Tungsten Deposit, Hunan Province. China Geol. 2021, 48, 1212-1224, (In Chinese with English abstract).

58. Whalen, J.B.; Kenneth, L.C.; Bruce, W.C. A-type granites, geochemical characteristics, discrimination and petrogenesis. Contrib. Mineral. Petrol. 1987, 95, 407-419. [CrossRef]

59. Wu, F.Y.; Jahn, B.M.; Wilde, S.A.; Lo, C.H.; Yui, T.F.; Lin, Q.; Ge, W.C.; Sun, D.Y. Highly fractionated I-type granites in NE China (I): Geochronology and petrogenesis. Lithos 2003, 66, 241-273. [CrossRef]

60. Ghaderi, M.; Palin, J.M.; Campbell, I.H.; Sylvester, P.J. Rare earth element systematics in scheelite from hydrothermal gold deposits in the Kalgoorlie Norseman region, Western Australia. Econ. Geol. 1999, 94, 423-438. [CrossRef]

61. Brugger, J.; Lahaye, Y.; Costa, S.; Lambert, D.; Bateman, R. Inhomogeneous distribution of REE in scheelite and dynamics of archaean hydrothermal systems (mt. charlotte and drysdale gold deposits, western australia). Contrib. Mineral. Petrol. 2000, 139, 251-264. [CrossRef]

62. Rudnick, R.L.; Gao, S. Treatise on Geochemistry. The Crust; Elsevier Science: Amsterdam, The Netherlands, 2004.

63. Huang, L.C.; Jiang, S.Y. Highly fractionated S-type granites from the giant Dahutang tungsten deposit in Jiangnan Orogen, Southeast China: Geochronology, petrogenesis and their relationship with W-mineralization. Lithos 2014, 202-203, 207-226. [CrossRef]

64. Li, W.S.; Ni, P.; Pan, J.Y.; Fan, M.S.; Chen, L.L.; Zhang, D.; Wu, X.W.; Gao, Y. Constraints on the timing and genetic link of scheeliteand wolframite-bearing quartz veins in the chuankou W ore field, South China. Ore Geol. Rev. 2021, 133, 104122. [CrossRef]

65. Rice, C.M.; Darke, K.E.; Still, J.W.; Lachowski, E.E. Tungsten-bearing rutile from the Kori Kollo gold mine, Bolivia. Mineral. Mag. 1998, 62, 421-429. [CrossRef]

66. Keppler, H. Influence of fluorine on the enrichment of high field strength trace elements in granitic rocks. Contrib. Mineral. Petrol. 1993, 114, 479-488. [CrossRef]

67. Li, Y.; Audétat, A. Effects of temperature, silicate melt composition, and oxygen fugacity on the partitioning of $\mathrm{V}, \mathrm{Mn}, \mathrm{Co}, \mathrm{Ni}, \mathrm{Cu}$, $\mathrm{Zn}, \mathrm{As}, \mathrm{Mo}, \mathrm{Ag}, \mathrm{Sn}, \mathrm{Sb}, \mathrm{W}, \mathrm{Au}, \mathrm{Pb}$, and Bi between sulfide phases and silicate melt. Geochim. Cosmochim. Acta 2015, 162, 25-45. [CrossRef]

68. Ishihara, S. The magnetite-series and ilmenite-series granitic rocks. Min. Geol. 1977, 27, 293-305.

69. Thompson, J.F.H.; Sillitoe, R.H.; Baker, T.; Lang, J.R.; Mortensen, J.K. Intrusion-related gold deposits associated with tungsten-tin provinces. Miner. Depos. 1999, 34, 323-334. [CrossRef]

70. Che, X.D.; Linnen, R.L.; Wang, R.C.; Aseri, A.; Thibault, Y. Tungsten solubility in evolved granitic melts: An evaluation of magmatic wolframite. Geochim. Cosmochim. Acta 2013, 106, 84-98. [CrossRef]

71. O'Neill, H.S.C.; Berry, A.J.; Eggins, S.M. The solubility and oxidation state of tungsten in silicate melts: Implications for the comparative chemistry of W and Mo in planetary differentiation processes. Chem. Geol. 2008, 255, 346-359. [CrossRef]

72. Wade, J.; Wood, B.J.; Norris, C.A. The oxidation state of tungsten in silicate at high pressures and temperatures. Chem. Geol. 2013, 335, 189-193. [CrossRef]

73. Li, C.Y.; Zhang, H.; Wang, F.Y. The Formation of the Dabaoshan Porphyry Molybdenum Deposit Induced by Slab Rollback. Lithos 2012, 150, 101-110. [CrossRef] 
74. Sun, Z.L. Geochronology and Oxygen Fugacity of Mesozoic Granitesin Nanling Area of South China. J. Earth Sci. Environ. 2014, 36, 141-150, (In Chinese with English abstract).

75. Blevin, P.L. Redox and compositional parameters for interpreting the granitoid metallogeny of eastern Australia: Implications for gold rich ore systems. Resour. Geol. 2004, 54, 241-252. [CrossRef]

76. Li, X.H.; Li, Z.X.; Li, W.X.; Wang, Y.J. Initiation of the Indosinian orogeny in South China, Evidence for a permian magmatic arc on the Hainan island. J. Geol. 2006, 114, 341-353. [CrossRef]

77. Chen, P.; Hua, R.; Zhang, B.; Lu, J.; Fan, C. Early Yanshanian post-orogenic granitoids in the Nanling region. Sci. China. Ser. D Earth Sci. 2002, 45, 755-768. [CrossRef]

78. Tapponnier, P.; Peltzer, G.; Le Dain, A.Y.; Armijo, R.; Cobbold, P. Propagating extrusion tectonics in Asia, new insights from simple experiments with plasticine. Geology 1982, 10, 611. [CrossRef]

79. Gilder, S.A.; Coe, R.S.; Wu, H.; Kuang, G.; Zhao, X.; Qi, W. Triassic paleomagnetic data from South China and their bearing on the tectonic evolution of the western circum-Pacific region. Earth Planet. Sci. Lett. 1995, 131, 269-287. [CrossRef]

80. Carter, A.; Roques, D.; Bristow, C.; Kinny, P. Understanding mesozoic accretion in southeast Asia, significance of Triassic thermotectonism. Geology 2001, 29, 211-214. [CrossRef]

81. Li, S.Z.; Jahn, M.; Zhao, S.J.; Dai, L.M.; Li, X.Y.; Suo, Y.H.; Guo, L.L.; Wang, Y.M.; Liu, X.C.; Lan, H.Y.; et al. Triassic southeastward subduction of North China Block to South China Block: Insights from new geological, geophysical and geochemical data. Earth-Sci. Rev. 2017, 166, 270-285. [CrossRef]

82. Sun, T.; Zhou, X.M.; Chen, P.R.; Li, H.M.; Zhou, H.Y.; Wang, Z.C.; Shen, W.Z. Genesis and tectonic significance of Mesozoic strong peraluminous granites in eastern Nanling area. Sci. China Earth Sci. 2003, 33, 1209-1218. (In Chinese)

83. Zhang, Y.Q.; Dong, S.W.; Li, J.H.; Cui, J.J.; Shi, W.; Su, J.B.; Li, Y. The New Progress in the Study of Mesozoic Tectonics of South China. Acta Geosci. Sin. 2012, 33, 257-279, (In Chinese with English abstract).

84. Gilder, S.A.; Keller, G.R.; Luo, M.; Goodell, P.C. Timing and spatial distribution of rifting in China. Tectonophysics 1991, 197, 225-243. [CrossRef]

85. Mao, J.W.; Xi, G.Q.; Li, X.F.; Zhang, C.Q.; Mei, Y.X. Meosozoic large scale mineralization and multiple lithospheric extension in South China. Earth Sci. Front. 2004, 11, 45-55, (In Chinese with English abstract).

86. Qin, J.H.; Wang, D.H.; Chen, Y.C. Geochemical and $\mathrm{Sr}-\mathrm{Nd}-\mathrm{Pb}$ isotopic characteristics of basalt from eastern Hunan Province: New insight for the Late Cretaceous tectonic dynamic mechanism in South China. Geol. J. 2020, 55, 8141-8168. [CrossRef]

87. Zhou, X.M. Genesis of Late Mesozoic Granite and Lithospheric Dynamic Evolution in Nanling Area; Science Press: Beijing, China, 2007; 691p, (In Chinese with English abstract).

88. Mao, J.W.; Xie, G.Q.; Guo, C.L.; Yuan, S.D.; Chen, Y.B.; Chen, Y.C. Spatial-Temporal Distribution of Mesozoic Ore Deposits in South China and Their Metallogenic Settings. Geol. J. China Univ. 2008, 14, 510-526, (In Chinese with English abstract).

89. Mao, J.W.; Pirajno, F.; Lehmann, B.; Luo, M.C.; Berzina, A. Distribution of porphyry deposits in the Eurasian continent and their corresponding tectonic settings. J. Asian Earth Sci. 2014, 79, 567-584. [CrossRef]

90. Shen, W.Z.; Ling, H.F.; Li, W.X.; Wang, D.Z. Sr and Nd isotope of Mesozoic granitoids in Jiangxi Province. Chin. Sci. Bull. 1998, 43, 2653. (In Chinese) [CrossRef]

91. Zhou, X.H. Multiple Isotope Constraint on Deep Processes of Continetal Lithosphere. Acta Geosci. Sin. 1997, 18, 1-2, (In Chinese with English abstract).

92. Chen, J.F.; Guo, X.S.; Tang, J.F.; Zhou, T.X. Nd isotope Model Ages, Implication of the Growth of the Continental Crust of Southeastern China. J. Nanjing Univ. 1999, 35, 649-658, (In Chinese with English abstract).

93. Wang, Y.; Guan, T.Y.; Liu, P.H.; Yu, D.J.; Wu, J.H. Nd and Sr Isopotic Geochemical Characteristics for Early Cretaceous Volcanic Rocks in Northeast Jiangxi Province. J. Mineral. Petrol. 2002, 22, 36-40, (In Chinese with English abstract).

94. Jiang, S.Y.; Peng, N.J.; Huang, L.C.; Xu, Y.M.; Zhan, G.L.; Dan, X.H. Geological characteristic and ore genesis of the giant tungsten deposits from the Dahutang oreconcentrated district in northern Jiangxi Province. Acta Petrol. Sin. 2015, 31, 639-655, (In Chinese with English abstract).

95. Huang, L.C.; Jiang, S.Y. Geochronology, geochemistry and petrogenesis of the tungsten-bearing porphyritic granite in the Dahutang tungsten deposit, Jiangxi Province. Acta Petrol. Sin. 2013, 29, 4323-4335, (In Chinese with English abstract).

96. Liu, Y.J.; Ma, D.S. The Geochemistry of Tungsten; Science Press: Beijing, China, 1987; pp. 1-222. (In Chinese)

97. Wang, D.H. Mantle Plume and Mineralization; Seismological Press: Beijing, China, 1998; pp. 1-160. (In Chinese)

98. Hua, R.; Chen, P.; Zhang, W.; Lu, J. Three large-scale metallogenic events related to the Yanshanian Period in Southern China. In Mineral Deposit Research: Meeting the Global Challenge; Mao, J., Bierlein, F.P., Eds.; Springer: Berlin/Heidelberg, Germany, 2005; pp. 401-404. [CrossRef]

99. Jiang, Y.H.; Wang, G.C.; Zheng, L.; Ni, C.Y.; Qing, L.; Zhang, Q. Repeated slab advance-retreat of the Palaeo-Pacific plate underneath SE China. Int. Geol. Rev. 2015, 57, 472-491. [CrossRef]

100. Jiang, Y.H.; Wang, G.C. Petrogenesis and geodynamics of late mesozoic granitoids in SE China: Tectonic model involving repeated slab-advance-retreat of the paleo-pacific plate. Bull. Mineral. Petrol. Geochem. 2016, 35, 1073-1081, (In Chinese with English abstract).

101. Zhou, X.M.; Sun, T.; Shen, W.Z.; Shu, L.S.; Niu, Y.L. Petrogenesis of Mesozoic granitoids and volcanic rocks in South China, a response to tectonic evolution. Episodes 2006, 29, 26-33. [CrossRef] 